\title{
Patterning of plant cell wall deposition by cortical microtubules
}

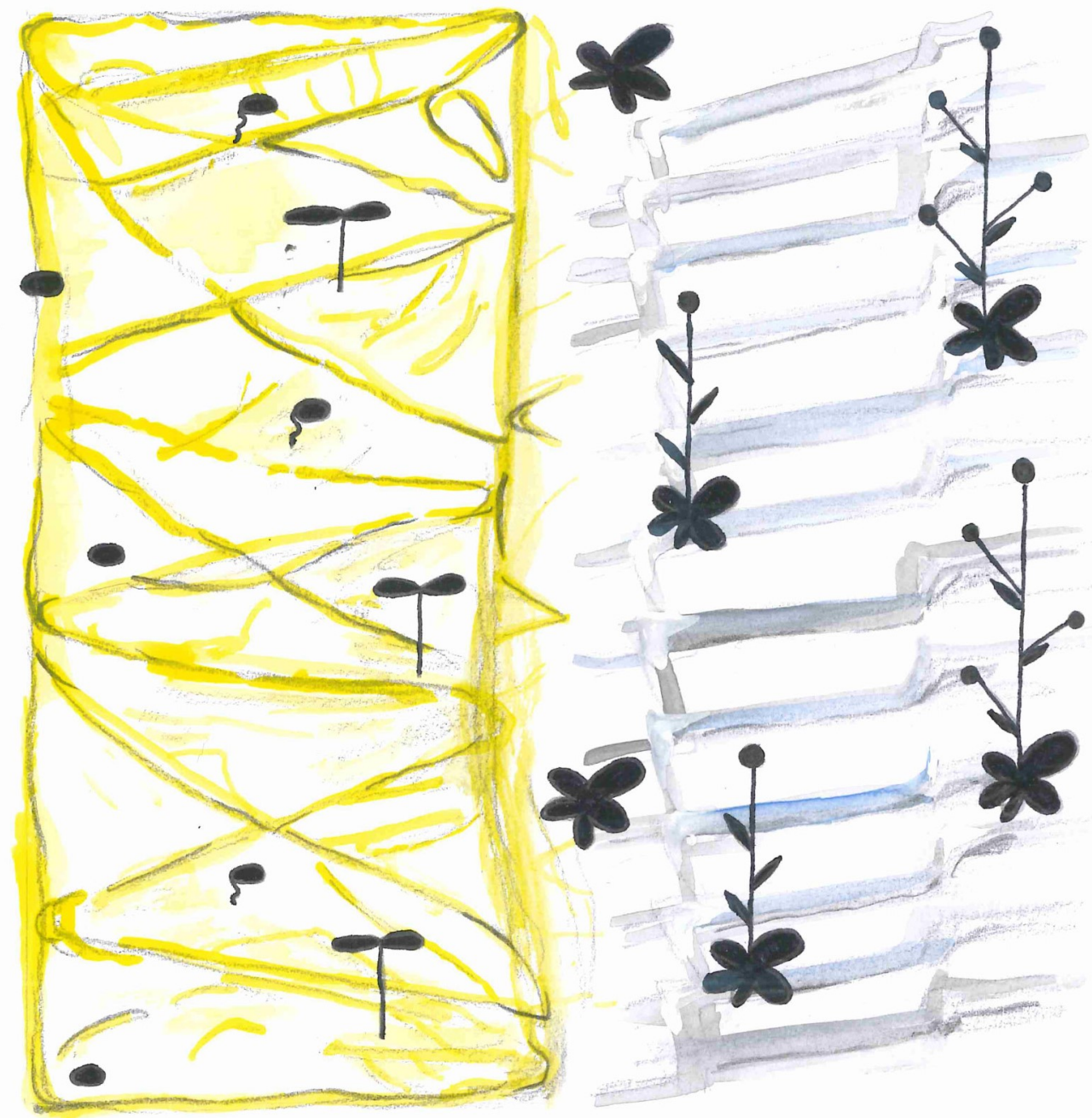

Kris Joppe van 't Klooster 


\section{Propositions}

1. Microtubule dynamics are more important for microtubule patterning than microtubule associated proteins (this thesis)

2. Cortical microtubules determine when and where secondary cell wall thickenings are deposited during protoxylem formation (this thesis)

3. Biological knowledge is temporal knowledge

4. New knowledge creates limiting boundaries in thinking

5. Artificial intelligence is a bigger threat to humanity than climate change

6. A scientist's mind can be nurtured, not created

7. To feed-back is to feed-forward

Propositions belonging to the thesis, entitled

Patterning of plant cell wall deposition by cortical microtubules

Kris Joppe van 't Klooster

Wageningen, 18 October 2017 


\section{Patterning of plant cell wall deposition by cortical microtubules}

Kris Joppe van 't Klooster 


\section{Thesis committee}

\section{Promotors}

Prof. Dr J. van der Gucht

Professor of Physical Chemistry and Soft Matter

Wageningen University \& Research

Prof. Dr B.M. Mulder

Special Professor Theoretical Cell Physics

Wageningen University \& Research

Group Leader Theory of Biomolecular matter Institute AMOLF, Amsterdam

\section{Co-promotors}

Dr M.J. Ketelaar

Assistant professor, Laboratory of Cell Biology

Wageningen University \& Research

Dring. J.J.W.A. van Loon

Senior researcher

VUMC \& ACTA, Amsterdam

\section{Other members}

Prof. Dr S.C. de Vries, Wageningen University \& Research

Dr M.J. Deeks, University of Exeter, United Kingdom

Prof. Dr G.H. Immink, Wageningen University \& Research

Dr V.A. Willemsen, Wageningen University \& Research

This research was conducted under the auspices of the Graduate School of Experimental Plant Sciences 


\title{
Patterning of plant cell wall deposition by cortical microtubules
}

\author{
Kris Joppe van 't Klooster
}

Thesis

submitted in fulfilment of the requirements for the degree of doctor at Wageningen University by the authority of the Rector Magnificus,

Prof. Dr A.P.J. Mol, in the presence of the

Thesis Committee appointed by the Academic Board to be defended in public on Wednesday 18 October 2017 at 11 a.m. in the Aula. 
Kris Joppe van 't Klooster

Patterning of plant cell wall deposition by cortical microtubules 114 pages.

PhD thesis, Wageningen University, Wageningen, the Netherlands (2017) With references, with summary in English

ISBN: 978-94-6343-622-9

DOI: $10.18174 / 419181$ 


\section{Contents}

\section{Chapter 1}

General Introduction

Chapter 2

Characterization of microtubule and secondary cell wall patterns during VND7-inducible protoxylem formation in Arabidopsis thaliana

\section{Chapter 3}

Local destabilization drives cortical microtubule rearrangement during protoxylem formation

Chapter 4

Spatial manipulation of microtubule catastrophe rate causes pattern formation of stochastically simulated microtubules

Chapter 5

Patterned secondary cell wall deposition during protoxylem formation proceeds normally under simulated altered gravity conditions

Chapter 6

General Discussion

Bibliography

Summary

Acknowledgements

Curriculum Vitae

Education Statement 



\section{Chapter}

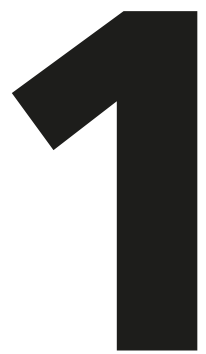

General Introduction 


\section{Xylem formation as experimental system to investigate patterning of plant cell wall deposition by cortical microtubules}

Plants are primary producers converting energy from the sun, carbon dioxide from the air and water from the soil into organic matter, which provides humanity with oxygen to breath, food to eat and wood as a versatile construction material. Seed plants are multicellular organisms in which cells differentiate to fulfil specific functions in the plant body. In this thesis, the experimental system of choice are the cells that function in water and nutrient transport, which is of cardinal importance for plant growth. This xylem tissue specializes in water and nutrient transport from root to shoot. The water-conductive components in the xylem are tracheids and vessel elements, together referred to as tracheary elements ${ }^{1}$. Each plant cell is surrounded by a primary cell wall which conveys structural support to the cell, and thereby to cell tissues and the plant as a whole'. Plant cell walls are biphasic structures, consisting of an interconnected matrix of the polysaccharides pectin, hemi-cellulose and cellulose microfibrils². The cellulose microfibrils are considered to be the load-bearing component in plant cell walls ${ }^{3,4}$. While cell wall matrix components are deposited against the existing cell walls by exocytosis, the cellulose microfibrils are synthesized by cell membrane resident CESA complexes ${ }^{5}$. The cell walls of tracheary elements have specific requirements. Water that evaporates at the leaves creates a negative water pressure in the xylem tissue, which is the driving force of water transport ${ }^{6}$. This negative pressure poses a risk of implosion of the tubular tracheary elements ${ }^{6}$. To overcome the risk of implosion, tracheary elements differentiate as elongated cells with reinforced cell walls ${ }^{7}$. The reinforcement of cell walls in tracheary elements is achieved by localized deposition of secondary cell wall thickenings, against the existing primary cell wall during differentiation (Fig. 1.1). Throughout evolution of tracheary elements, incremental adaptations have greatly increased the conductivity of this tissue while at the same time limiting the risk of tracheary implosion ${ }^{6}$. Conductivity is further improved by perforations of the top and bottom of vessel forming tracheary element ${ }^{6}$, resulting in the formation of straw-like vessels (Fig. 1.1). As a final process in differentiation of tracheary elements, the cells undergo programmed cell death, which removes the protoplast and thereby also improves conductivity ${ }^{7}$. 


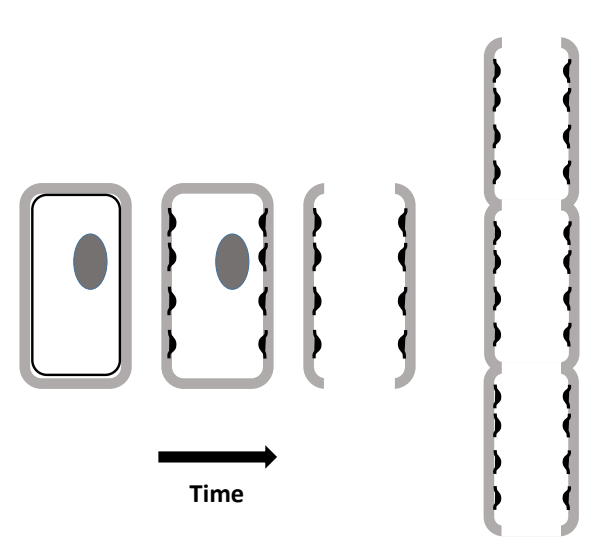

Figure 1.1 | Schematic representation of protoxylem formation. A longitudinal cross section of a simplified plant cell is depicted with a primary cell wall (grey), a plasma membrane and a nucleus (left). Additional cell wall material is deposited in rings (black protuberances), which causes plasma membrane invagination. Furthermore, the top and bottom of the primary cell wall degrade to improve conductivity of the tracheary element. Multiple tracheary elements connected form a tubular transport system (right).

\section{Patterns of secondary cell wall thickenings in protoxylem and metaxylem}

Seed plants can be divided into two groups, the gymnosperms and the angiosperms. In gymnosperms seed development takes place "naked" whereas in angiosperms the seed development occurs covered within fruit. The xylem tissue of gymnosperms consists of tracheids only, whereas xylem tissue of angiosperms consists of tracheids, vessel elements and libriform cells ${ }^{1}$. The diameter of tracheids is smaller than vessel elements and thereby tracheids provide more structural support and less conductivity compared to vessel elements. Besides a wider diameter, conductivity in vessel elements is higher than in tracheids because the end walls of individual cells are removed during vessel element differentiation, resulting in continuous tubes consisting of multiple vessel elements ${ }^{8}$. These characteristics of vessel elements provide less structural support. This can be compensated by libriform cells, elongated fibre like cells specialized for structural support via secondary cell wall reinforcement ${ }^{7}$. Whereas libriform fibres have a uniformly deposited secondary cell wall, tracheary elements have patterned secondary cell wall thickenings. Besides structural support for the plant as a whole, these patterned secondary wall thickenings resist forces during transport, preventing collapse of the vessel as mentioned previously ${ }^{6,9-12}$. Secondary cell wall thickenings in tracheary elements are deposited in different patterns, such as

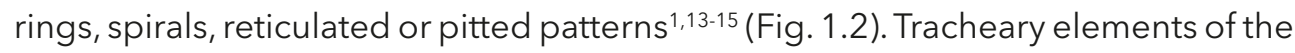
protoxylem undergo terminal differentiation before cell growth of the surrounding tissues has ceased ${ }^{1,7}$. After programmed cell death, the reinforced cell walls of protoxylem elements need to be able to stretch to accommodate the elongation of the surrounding cells $s^{1,7}$. Ring and spiral shaped cell wall thickenings have this capacity (Fig. 1.2AB). Tracheary elements of the metaxylem undergo their terminal differentiation program after elongation of the surrounding tissues has ceased. 
Therefore, the cell wall reinforcements in this tissue do not require the ability to stretch. Typical secondary cell wall thickenings of tracheary elements of the metaxylem are the reticulated and pitted patterns which provide more structural support than protoxylem patterns but are incompatible with tissue elongation (Fig. 1.2CD). To study patterned deposition of secondary cell wall thickenings during xylem differentiation, we focus on protoxylem vessel elements because patterning of rings and spirals is more predictable and less complex compared to the patterning of reticulated and pitted cell wall thickenings of the metaxylem vessel elements.
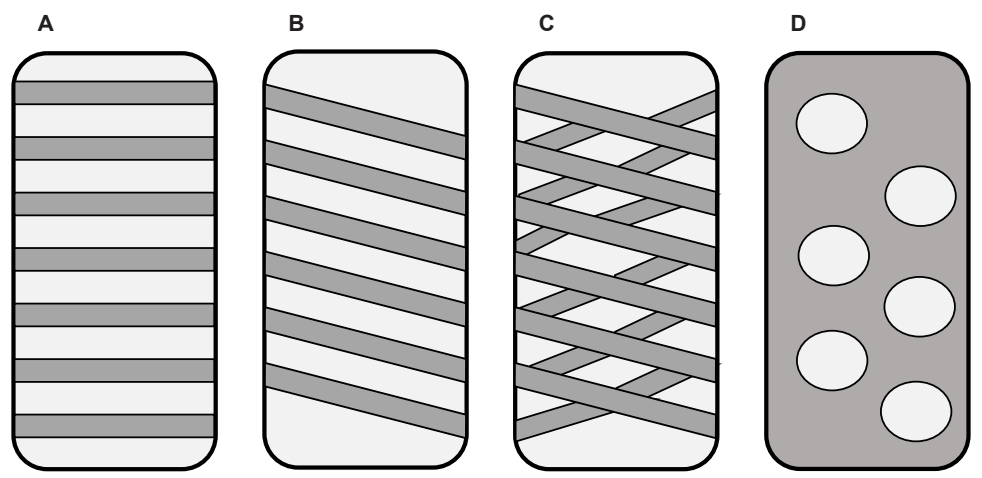

Figure 1.2 | Different types of secondary cell wall thickenings in tracheary elements. In light grey the primary cell wall is depicted and in dark grey different types of secondary cell wall thickenings. Typical for tracheary elements of the protoxylem are the ring (A) and spiral (B) patterns, whereas reticulated (C) and pitted (D) patterns are typical for tracheary elements of the metaxylem. Ring and spiral patterns do provide structural allowing surrounding tissue to elongate, whereas reticulated and pitted patterns provide more support but prevent elongation of surround tissue.

\section{Patterns in nature}

Before I discuss the current understanding of pattern formation during xylem formation it is necessary to first address the question: what is a pattern? Then, I will address how plant cells can form a pattern via a reaction-diffusion mechanism, what the functionalities are of the microtubule array and how a patterned microtubule array is translated to patterned deposition of secondary cell wall thickenings. Cambridge's dictionary definition of the word 'pattern' is: any regularly repeated arrangement, especially a design made from repeated lines, shapes, or colours on a surfacei. Patterns occur at different length scales within nature. Recently Tarnita et al. (2017) showed that interaction among social-insect colonies and vegetation can create regional vegetation patterns ${ }^{16}$. Also at a smaller scale patterns emerge, such as the min-system in Escherichia coli in which oscillating proteins mediate 
bacterial cell division ${ }^{17}$. In plants, the positioning of veins throughout leaves may be patterned. Plant veins contain the transport tissues phloem, which transport the nutrients produced by photosynthesis throughout the plant and xylem, which, as discussed above, transports water and nutrients taken up by the roots of plants and consists of tracheary elements with patterned secondary cell wall thickenings. Thus, patterning occurs in a wide variety of systems in nature that largely differ in size. In the next paragraph, I will address general mechanisms that underlie patterning before I focus on the specific case of patterned secondary cell wall thickenings in tracheary elements.

\section{Mechanisms that underlie pattern formation}

Patterns can originate externally or internally within a system. External pattern originates from a template outside of the studied system. The layout of a farm is an external example, where a farmer enforces a farm layout. Internal patterns can emerge from within the system itself. Self-organization is a mechanism by which internal patterns emerge without the need for a prescribed template ${ }^{18}$. Selforganizing principles can be based on the principle of positive feedback loops on the local scale and negative feedback loops on a bigger scale ${ }^{19}$. The simplest form of a pattern forming self-organization mechanism is the Turing's reactiondiffusion model, which consists of a slowly diffusing activator that recruits more activator and a rapidly diffusing deactivato ${ }^{20}$. Local concentration differences may result in an excess of activator, which will locally enhance the reaction. If the diffusion rate of the deactivator is faster than the activator, this will constrain the location at which the activator can enhance the reaction. Thereby this mechanism may result in the spatial separation of the activator and the deactivator depending on the reaction and diffusion rates ${ }^{20}$. This can lead to a spatially stable pattern or dynamics patterns which oscillates or travels in waves ${ }^{21}$. If the interacting molecular components of a pattern forming system are identified, the sum of the interactions of the components is often not sufficient to understand the output of a system. Mathematical models help to investigate the possibilities how the interacting components may result in a certain pattern. Simulations that are based on Turing's reaction-diffusion model have been used to truthfully reproduce fish skin patterns, and more importantly, predicted how this pattern would change upon a disturbance during establishment of the skin pattern ${ }^{21}$. Mathematical models can thereby be of great use as they provide the possibility to test existing hypotheses and generate new hypotheses 22,23 . These hypothesises can guide biological experiments. Simulations can also be used to test ideas which are practically not executable or expensive. The iterative combination of mathematical models with biological experiments provides a powerful tool to generate new knowledge. 


\section{Mechanisms that underlie microtubule reorganization}

Before I discuss the knowledge about how cortical microtubules template cellulose deposition during secondary cell wall thickenings, I will first focus on the question 'what mechanisms underlie microtubule reorganization?'. Microtubules are filamentous protein polymers consisting of alpha- and beta-tubulin ${ }^{24}$, which are conserved in eukaryotic organisms, including plants ${ }^{25}$. Heterodimers of alpha- and beta-tubulin subunits form protofilaments, which assemble into a tubular structure with a diameter of $24 \mathrm{~nm}^{24}$. Nucleation complexes assist in the initial assembly of a microtubule ${ }^{26}$. Microtubules are polar structures due to the directionality imposed by the alpha- and beta-tubulin subunits, with alpha- and beta-tubulin heterodimer addition preferentially occurring at the plus end ${ }^{24}$. The minus end can remain connected to a nucleation complex, or the minus end is severed and can then slowly depolymerise ${ }^{26}$. The plus end stochastically switches between phases of growth (polymerization) and shrinkage (depolymerization) via catastrophes and rescues ${ }^{26}$. The switch from a growing to a shrinking state of a microtubule is defined as a catastrophe and the switch from shrinking to growing as a rescue ${ }^{27}$. Microtubules that stochastically switch between growing and shrinking states are considered to be dynamic instable ${ }^{26,28}$ (Fig. 1.3). Dynamic instability emerges from the mechanism of microtubule polymerization ${ }^{29,30}$. Newly added tubulin heterodimers contain GTP which stabilizes the plus end of a microtubule, over time the GTP hydrolyses into GDP which cannot stabilize the microtubule and thus a catastrophe will follow 29,30. As long as the rate of polymerization is faster than the GTP hydrolysis, a GTP-cap remains at the plus end of the microtubule preventing a catastrophe $e^{29,30}$.

In interphase plant cells, microtubules can be attached to the plasma membrane via anchoring proteins ${ }^{31}$, and are then defined as cortical microtubules. The collection of dynamic microtubules attached to the plasma membrane is the cortical microtubule array. Nucleation complexes are usually located on existing microtubules and nucleate new microtubules in a parallel or antiparallel with the existing microtubules, or in a predominant angle of approximately $40^{\circ}$ compared to the mother microtubule $\mathrm{e}^{32,33}$. The connection with the plasma membrane affects how dynamic cortical microtubules interact within the array as the freedom of movement is effectively restricted to a two-dimensional plane ${ }^{34}$. The angle of one microtubule growing into another microtubule determines the chance by which it will cross-over the already existing microtubule, or if it will undergo an induced catastrophe ${ }^{34}$. These dynamic characteristics of cortical microtubules, together with the possibility of new microtubule nucleation, allow the array to self-organize ${ }^{35}$. The dynamic instability characteristics of the microtubule array, nucleation, growth \& shrinkage velocity and catastrophe \& rescue rate, can be regulated by microtubule associated proteins (MAPs) ${ }^{36,37}$. Thus, the intrinsic 
dynamic instability of microtubules in the plant cortical array and the sum of activities of MAPs determines the dynamic properties of the array. The outcome of this process is patterning of the cortical array governed by self-organisation.

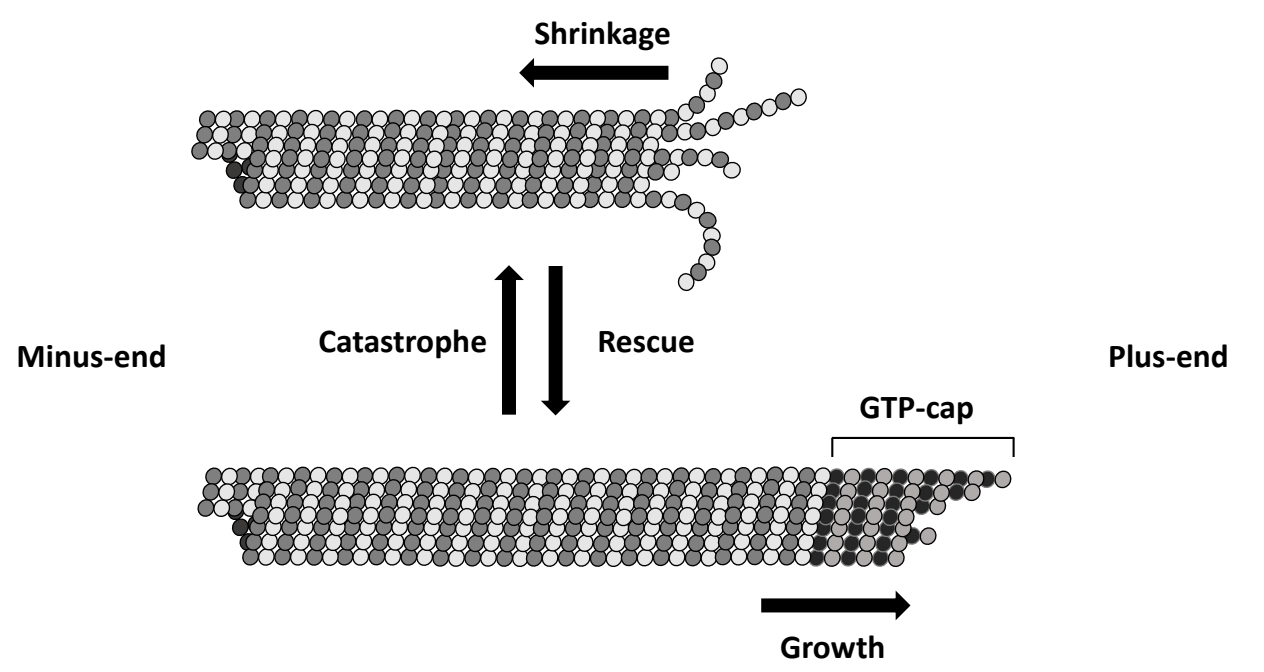

Figure 1.3 | Dynamic instability properties of microtubules. Microtubule polymers stochastically switch between growing and shrinking states which are defined as a catastrophe for the switch from a growing to a shrinking state, and a rescue for the switch from a shrinking to a growing state. The light and dark grey subunits represent the alpha- and beta-tubulin. The minus end is at the left side and the growing or shrinking plus end is on the right side. The growing plus end has a GTP-cap which stabilises the microtubule. Image adapted from Bowne-Anderson et al. (2013) ${ }^{30}$.

\section{Cortical microtubules direct cellulose microfibril deposition}

The patterning of cortical microtubules is important, since the cortical microtubules control the direction of cellulose microfibril deposition ${ }^{38-41}$. During cell wall deposition cellulose synthase (CesA) complexes move through the plasma membrane depositing cellulose microfibrils ${ }^{42}$. The CesA complexes which reside in the plasma membrane acquire UDP-glucose from the cytosol and synthesise the polymeric cellulose microfibril which are deposited between the plasma membrane and the existing cell wall ${ }^{42}$. The movement of CesA complexes through the plasma membrane is thought to be driven by the force that is generated by the addition of UDP-glucose molecules to cellulose microfibrils ${ }^{43}$. Although cortical microtubules do not provide the force for CesA complex motility per se, CesA complexes are preferentially inserted into the plasma membrane at locations where cortical microtubules reside and since cortical microtubules are attached to the plasma membrane, they template the motility direction of CesA complexes and thus of cellulose microfibril deposition ${ }^{38-40}$. Cells are able to control the orientation in which cellulose is deposited ${ }^{38}$, but are also able to modify the orientation of 
the cortical microtubules, and thus of cellulose deposition and cell growth, in response to environmental cues, such as light ${ }^{44}$. The study of the microtubule array therefore can be used as a proxy for studying the location and orientation of cellulose microfibril deposition into the plant cell wall matrix ${ }^{41}$.

\section{Transdifferentiation systems to study xylem formation}

Until recently, knowledge about microtubule organisation and dynamics during xylem formation was patchy, mainly because (1) sequential differentiation makes it difficult to identify cells which are in a similar developmental stage and (2) there is limited accessibility by light microscopy as the xylem differentiates in the central stele which is covered by layers of epidermal and cortex plant cells. To overcome these difficulties transdifferentiation systems have been used to study xylem formation. In these systems, non-xylem plant cells can be triggered to transdifferentiate into xylem-like cells by hormonal treatment ${ }^{45,46}$. Using trans-differentiation systems, xylem formation has been studied in cell cultures of Zinnia elegans ${ }^{46-48}$ and Arabidopsis thaliana (Arabidopsis) ${ }^{49,50}$. These studies resulted in the discovery of the master regulators of proto- and metaxylem formation Vascular NAC Domain 7 (VND7) and VND6 respectively in Arabidopsis ${ }^{45}$. An Arabidopsis transdifferentiation system was created by combining the master regulators for xylem formation with a chemically inducible glucocorticoid-receptor ${ }^{51}$. Induction of this transdifferentiation system by applying the synthetic glucocorticoid dexamethasone, results in the simultaneous ectopic transdifferentiation of many cells in the plant body into xylem-like cells ${ }^{51}$.

\section{A molecular mechanism regulating pattern formation in metaxylem}

Like in primary cell walls, microtubules have been demonstrated to template cellulose deposition during the formation of secondary cell wall thickenings ${ }^{52}$. Recent studies focussed on the formation of a pitted pattern of secondary cell wall thickenings of the metaxylem ${ }^{53-55}$. Upon induction of VND6, a molecular pathway is activated which patterns the membrane bound GTPase ROP11 in Arabidopsis ${ }^{53}$. ROP11 is activated and deactivated by ROPGAP3 and ROPGEF4, respectively, which have been hypothesized to be patterned via a reaction-diffusion mechanism ${ }^{53}$ (Fig. 1.4). Activated ROP11 ${ }^{\mathrm{GTP}}$ interacts with the MAPs MIDD1 and AtKinesin13A, which jointly depolymerizes cortical microtubules from the plasma membrane in a domain with active ROP11 GTP 54,55 (Fig. 1.4). Cortical microtubules have been shown to act as a barrier to membrane bound ROPP11 GTP-MIDD1 in in leaf epidermal cells of Nicotiana benthamiana ${ }^{53}$. It is unclear if and how this barrier function of microtubules affects metaxylem pattern formation in Arabidopsis. Lacking the cortical microtubules that template secondary cell wall thickenings ${ }^{52,56}$, these local activated ROP11 GTP areas remain free of additional deposition of secondary 
cell wall material, resulting in the characteristic pitted pattern of the metaxylem ${ }^{53}$ (Fig. 1.4). This indicates that microtubule dynamics need to be adjusted for xylem pattern formation to occur in a differentiating cell.

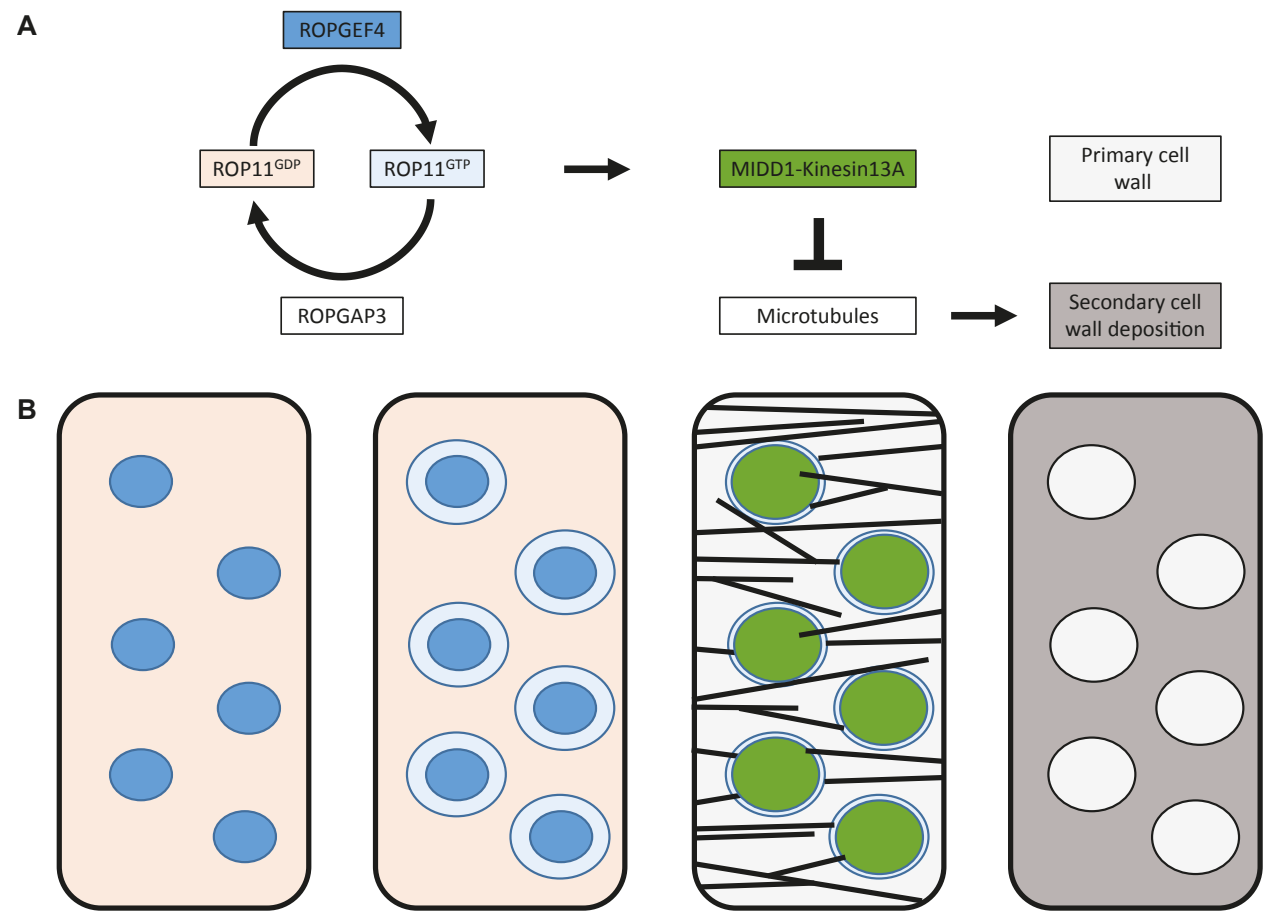

Figure 1.4 | Model to generate the pitted pattern typical of metaxylem vessel elements. A reaction-diffusion mechanism is proposed between ROPGEF4 and ROPGAP3 which activate and deactivate the GTPase ROP11 (A) and thereby creates spaced ROP11 GTP plasma membrane domains (B). Active ROP11 ${ }^{\mathrm{GTP}}$ attracts the microtubule associated proteins MIDD1 and AtKinesin13A to active ROP11 plasma membrane domains. The combination of MIDD1AtKinesin $13 \mathrm{~A}$ remove cortical microtubules from these ROP $11^{\text {GTP }}$ domains. Cortical microtubules guide secondary cell wall machinery to the plasma membrane, thus at the ROP $11^{\text {GTP }}$ domains, no secondary cell wall is deposited resulting into the characteristic pitted pattern of the metaxylem. This model is based on work by Oda \& Fukada (2010-2013) $)^{53-55,57}$.

\section{Research question and hypothesis}

The ROP11-MIDD1 pathways provides insights into the molecular components which generate the pitted pattern of the metaxylem, but a knockout of the ROPGEF4 activator of ROP11 or the expression of a constitutively active ROP11 did not affect spiral secondary cell wall thickenings in the protoxylem ${ }^{53}$. During the initial stages of my research, I have tried to identify alternative ROP pathways active in protoxylem formation (see discussion) but, as we were not able to detect protoxylem patterning phenotypes in individual ROP knockouts, we focused on investigation of the microtubule dynamics during protoxylem formation independently from the 
underlying molecular mechanisms. Understanding of the microtubule dynamics during protoxylem formation can aid in the discovery of the molecular components. The main research question addressed in this thesis is: which self-organizing properties of microtubules change to generate a microtubule pattern and how is this pattern translated to a patterned cell wall deposition during protoxylem formation? We assume that microtubule dynamics are crucial for patterned deposition of secondary cell wall thickenings during protoxylem formation and hypothesise that microtubule dynamics change resulting in a patterned microtubule array.

\section{Outline of this thesis}

In chapter 2 we characterized the changes in the microtubule array and patterned deposition of secondary cell wall thickenings during protoxylem formation. To study protoxylem formation in living plants using microscopy techniques, we used the ectopic differentiation system described by Yamaguchi et al. (2010) ${ }^{51}$, that uses inducible expression of the VND7 master regulator of protoxylem formation in Arabidopsis ${ }^{45}$. We used this VND7-system to reproducibly induce protoxylem formation in cortical cells into protoxylem-like vessel cells accessible for microscopy. We investigated this inducible system to determine the time scale of microtubule array reorganization and the connection between the pattern of the microtubule array and the localized deposition of secondary cell wall thickenings.

Based on our characterization of this inducible system described in chapter 2, we zoomed in on microtubule reorganization from the cell scale to the microtubule scale. Since the microtubule array templates the position of secondary cell wall thickenings, microtubule reorganization is a critical step in this patterning process ${ }^{52-54}$ (chapter 2). To understand biological processes depending on the microtubule array, we need to understand microtubule dynamics, as microtubules in the array are constantly in motion ${ }^{26}$. In chapter 3, we asked what changes in microtubule dynamics underlie the microtubule array reorganization observed during protoxylem formation. We showed that localized changes in rescue and catastrophe rates occur during microtubule reorganization. In areas that eventually will differentiate into secondary cell wall thickenings, the microtubule band zones, we observed no changes. The areas between future secondary cell wall thickenings, the microtubule gap zones, showed increased catastrophe and rescues rates. Quantification of the growth and shrinkage velocities did not show a difference locally or over time in microtubule band zones or gap zones. From these microtubule parameters a mean microtubule length was calculated, which resulted in a reduced calculated mean length in microtubule gap zones in between microtubule band zones with an unchanged calculated mean length. 
To test the relevance of the experimental parameters obtained in chapters 2 \& 3 , we tested the hypothesis that the changes in microtubule dynamics we observed are sufficient for band formation to occur. Therefore we created a stochastic simulation in chapter 4 . From previous microtubule simulation studies we know that a 2D microtubule array in plants can self-organize depending on the microtubule dynamicity parameters growth velocity, shrinkage velocity, rescue rate, catastrophe rate and nucleation rate ${ }^{58,59}$. These parameters form the foundation of the stochastic simulation and we manipulate the catastrophe rate to mimic the experimentally observed reorganization of the microtubule array during protoxylem formation ${ }^{60}$ (chapter 2 \& 3). By applying a template of alternative high and low catastrophe rate, we can recreate the experimentally observed microtubule pattern. This affects the mean length of the microtubules in a non-uniform way, resulting in a pattern with a high and low density of microtubules and is sufficient for microtubule band formation. This stochastic microtubule simulation helps to understand our observations, can be used to test regimes that cannot be tested experimentally and can suggest additional experiments.

In the previous chapters we generated mechanistic knowledge about pattern formation during protoxylem formation. In chapter $\mathbf{5}$ we test if gravity is a factor that contributes to this process. It has been suggested that a reaction-diffusion mechanism could be functioning during xylem formation ${ }^{53}$. In reaction-diffusion systems bifurcation points can exist ${ }^{61,62}$, at which a small change in circumstances can greatly affect the final outcome. Gravity has been suggested to be a force that can affect microtubules ${ }^{63-65}$ and microtubules have been suggested to form diffusion boundaries during metaxylem formation ${ }^{53}$. We mimicked altered gravity conditions by incubating samples on a random positioning machine and a centrifuge at the research facilities of the European Space Agency (ESA; ESTEC, Noordwijk, $\mathrm{NL}$ ). During this incubation, we ectopically induced VND7 activity to induce protoxylem transdifferentiation in seedlings experienced simulated microgravity and hypergravity. Long term and high quality visualization of the microtubule array in living plant cells as done in chapter $2 \& 3$, is technically challenging during the altered gravity treatments. Therefore, in chapter 5 we focussed on the output of the protoxylem differentiation program: the localized cell wall thickenings, which can be seen as a readout of the reorganization of the microtubule array during protoxylem formation. Since we do not find significant changes in band width and band distance between secondary cell wall thickenings, we conclude that our data does not support the hypothesis that altered gravity conditions can affect the microtubule array reorganization during protoxylem formation. 



\section{Chapter

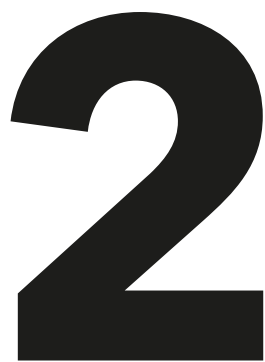

Characterization of microtubule and secondary cell wall patterns during VND7-inducible protoxylem formation in Arabidopsis thaliana 


\section{Introduction}

Patterns are observed in nature at many different levels. Here we study patterning at the plant cell level of vessel elements in Arabidopsis (Arabidopsis thaliana). The xylem is part of the vascular tissue and is responsible for transport of water, nutrient and signalling molecules throughout the plant. Vessel elements of the xylem are specialized transport cells that form a tubular system from root to shoot in a plant. During the evolution of xylem there has been a constant trade-off between optimization of conductivity and mechanical strength of vessel elements ${ }^{6}$. To optimise mechanical strength, vessel elements developed secondary cell wall thickenings in intricate patterns to reinforce the existing primary cell wall. Secondary cell wall thickenings in vessel elements can be in the form of rings, spirals, a reticulated or a pitted pattern ${ }^{13}$. After vessel elements are reinforced by secondary cell wall thickenings, the vessel element cells undergo programmed cell death to create a lumen which increases the conductivity ${ }^{66}$. We study how the differentiating protoxylem vessel element cells create patterned secondary cell wall thickenings. The plant cell wall is a structure that consists of strong, linear cellulose microfibrils embedded in a cell wall matrix, a structure that is similar to that of reinforced concrete ${ }^{3}$. Cell wall deposition is at least in part guided by cortical microtubules, both in the primary cell wall that is deposited during cell expansion ${ }^{38,67-69}$ and in the secondary cell wall that is deposited after cell growth has ceased ${ }^{52}$. Thus, cortical microtubules are important in determining the location and orientation of cellulose microfibrils at the plant cell level.

The cortical microtubule array consists of dynamic microtubules which stochastically switch from growing to shrinking states and vice versa. These switching events are called catastrophes and rescues, respectively. Microtubule arrays occur in different patterns. The type of pattern is governed by the microtubule dynamics and by the boundary conditions imposed by the shape of the cell. The dynamics of microtubules are regulated by microtubule associated proteins (MAPs), which can affect nucleation, severing, bundling and growth rates of single microtubules, thereby influencing the organization of the microtubule array ${ }^{36,37}$. How MAPs can affect the microtubule array during xylem differentiation is well studied in the formation of the pitted cell wall in metaxylem vessel elements. The location of future secondary cell wall thickening pits in metaxylem vessel elements are devoid of microtubules during the cell wall deposition ${ }^{53}$. Microtubule patterning in the metaxylem is mediated by the Rho GTPase ROP11. Active ROP11 is localized to the future pit regions where it recruits MIDD1, a MAP that suppresses rescue events ${ }^{53}$. Furthermore, AtKinesin13A, which is known to depolymerise microtubules, is recruited to the future pit regions ${ }^{54}$. The combined actions of MIDD1 and AtKinesin13A result in the formation of regions without cortical 
microtubules and thus without secondary cell wall deposition ${ }^{53-55}$. ROP11 does not appear to be involved in the patterning of secondary cell wall thickenings in protoxylem vessel elements ${ }^{53}$, however it is possible that a ROP-based mechanism is facilitating patterning in differentiating protoxylem vessel elements. Here we focus on the microtubule array and deposition of secondary cell wall thickenings during protoxylem vessel differentiation.

How is patterning, deposition of cell wall material and programmed cell death organized in time? How is the microtubule array patterned and what is the connection between the microtubule array and deposition of secondary cell wall thickenings during protoxylem vessel formation in space and time? Microscopic analysis of vessel element differentiation is complicated by the fact that vessel elements have a central location in plant organs, surrounded by a number of cell layers, including the cortex and epidermis. High resolution live-cell microscopy of vessel elements is therefore challenging. An additional complicating factor is that differentiation of the procambial cells into vessel elements occurs one by one over time as a plant grows. Therefore a limited number of differentiating vessel elements is present at a given time point and each of the cells is in a different developmental stage. The identification of the master regulators of the xylogenesis developmental program, Vascular-Nac-Domain-6 and VascularNac-Domain7 (VND6 \& VND7) 45 , has allowed the development of plant systems in which xylogenesis can be induced in any cell type at any moment ${ }^{51}$. We used an Arabidopsis line in which VND7 expression, which triggers protoxylem formation ${ }^{45}$, driven by the constitutive Cauliflower Mosaic Virus $35 \mathrm{~S}$ promotor is induced by dexamethasone (DEX) application ${ }^{51}$. In this line we have focused on transdifferentiating epidermal cells, which are suitable for microscopy, to study patterning. To investigate the interaction between microtubule dynamics and the deposition of secondary cell wall thickenings, microtubule drugs have been used during protoxylem differentiation in cell lines that are induced to transdifferentiate by DEX treatment.

Depolymerisation of microtubules by colchicine causes abnormal secondary cell wall patterns in vessel element development ${ }^{70,71}$. Oda et al. (2005) described roughening of the rims of secondary cell wall thickenings while the overall pattern of secondary cell wall deposition did not change due to microtubule depolymerisation by colchicine or propyzamide, applied when secondary cell wall thickenings just became visible ${ }^{72}$. Microtubule depolymerisation during earlier stages caused disordered and irregular secondary cell wall thickenings ${ }^{14,55}$. Microtubule stabilization by taxol at the start of vessel element development results in simpler patterns and a change in orientation from transverse to the long axis of the cell to oblique or longitudinal orientations of the cell wall thickenings ${ }^{14,47,72}$. 
Oda et al. (2005) further reported a reduced spacing between secondary cell wall thickenings due to stabilization of microtubules during vessel element development ${ }^{72}$. These results suggest that microtubules are involved both in the pattern establishment and the deposition of secondary cell wall during vessel element differentiation.

Using an Arabidopsis line carrying the VND7 inducible expression system described above ${ }^{51}$, we have studied the patterning of microtubules and regulated deposition of secondary cell wall thickenings. We have applied microtubule stabilizing and destabilizing drugs to assess the importance of microtubule dynamics on secondary cell wall thickening patterning over time in protoxylem formation. We show that the microtubule array reorganizes into a banded pattern which is followed by the localized deposition of secondary cell wall thickenings. Understanding the cell developmental processes during protoxylem formation both in space and in time provides a basis for further detailed studies in the interrelation between microtubules, deposition of secondary cell wall thickenings and cell patterning in general.

\section{Materials and Methods}

\section{Plant material and growth conditions}

Arabidopsis (Arabidopsis thaliana, Columbia Col-0 ecotype) plants expressing a $35 S$ promoter-driven VND7-VP16-GR construct (VND7), or a 35S promoter-driven VP16-GR empty vector control construct (EV) or the VND7 and EV lines expressing a $35 \mathrm{~S}$ promoter-driven YFP-TUA5 ${ }^{44}$ were used. For all lines homozygous F3 progeny was used. Seeds were surface-sterilized by application of a solution of $1 \%$ bleach (Sodium hypochlorite) and $0.1 \%$ Tween 20 for 10 min followed by thorough washing with sterile water, then stratified for a minimum of two days at $4^{\circ} \mathrm{C}$ in the dark. Seeds were grown either on Hoagland's modified basal salt mixture (Phyto Technology Laboratories, Kansas - USA, $1 \%$ agarose, pH 5.7), or on 0.5 Murashige and Skoog (MS, Sigma-Aldrich, $0.68 \%$ agarose, $\mathrm{pH} 5.7$ ) medium supplemented with $1 \%(\mathrm{w} / \mathrm{v})$ sucrose. Plates were placed vertically in a long-day phytotron $\left(25^{\circ} \mathrm{C}, 16 / 8 \mathrm{~h}\right.$ photoperiod).

\section{Induction of protoxylem formation}

VND7-induction was performed by transferring 5-day-old seedlings from the agarose plates to multi-well plates containing $10 \mu \mathrm{M}$ dexamethasone (DEX; 20 $\mathrm{mM}$ stock solution) in water. The multi-well plates were placed on a rotary shaker at $120 \mathrm{rpm}$ in a long-day phytotron $\left(25^{\circ} \mathrm{C}, 16 / 8 \mathrm{~h}\right.$ photoperiod $)$. Oryzalin treatments 
were performed by transferring 5-day-old seedlings from agarose plates to multiwell plates containing $10 \mu \mathrm{M}$ DEX in water and placed on a rotary shaker. For ' $3 \mathrm{~h}$ ' oryzalin-treatments $10 \mu \mathrm{M}$ oryzalin was added to the seedlings $3 \mathrm{~h}$ prior to DEXtreatment. For ' $6 \mathrm{~h}-48 \mathrm{~h}$ ' oryzalin-treatments the seedlings were incubated with 10 $\mu \mathrm{M}$ oryzalin $6 \mathrm{~h}, 12 \mathrm{~h}, 24 \mathrm{~h}$ or $48 \mathrm{~h}$ after the start of DEX-treatment. Taxol treatment was performed by treating 3-day-old seedlings with $20 \mu \mathrm{M}$ taxol and $10 \mu \mathrm{M}$ DEX in water. For the cell wall analysis experiments, wild-type, VND7, and the EV plants were grown on a rotary shaker at $100 \mathrm{rpm}$ in liquid $0.5 \mathrm{MS}$ medium supplemented with $1 \%$ sucrose at $\mathrm{pH} 5.7$ in the dark. After 5 days, the cultures were treated with $10 \mu \mathrm{M}$ DEX together with $10 \mu \mathrm{M}$ oryzalin or $20 \mu \mathrm{M}$ taxol in water and were collected after an incubation of 2 days in the dark.

\section{Staining}

Cell wall staining was performed by transferring seedlings to either $0.5 \mu \mathrm{g} \mathrm{mL}^{-1}$ propidium iodide (Sigma-Aldrich, MO, USA) in water for 10 minutes followed by a 5 minute wash in sterile water, or to $0.02 \%$ Direct Red 23 (Sigma-Aldrich, MO, USA) in water for 30 minutes followed by exchange of the medium by sterile water. Live-dead staining was performed by treatment with $10 \mu \mathrm{M}$ fluorescein diacetate (FDA, Sigma).

\section{Microscopy}

Imaging was performed on either an Axiovert 200M microscope (Zeiss, Jena, Germany) connected to a Zeiss LSM510 META confocal scanning system equipped with a $40 x$ (N.A. 1.3) and $63 x$ (N.A. 1.4) oil immersion objectives linked to a $30 \mathrm{~mW}$ Ar laser $(488 \mathrm{~nm})$ and a $1 \mathrm{~mW}$ HeNe laser $(543 \mathrm{~nm})$, or a spinning disc confocal microscope consisting of a CSU-X1 spinning disk head (Yokogawa) attached to an Eclipse TI inverted microscope (Nikon) equipped with a perfect focus system, an Evolve CCD camera (Photometrics), a Plan Apo 100X/1.4 NA oil immersion objective and a $1.2 \mathrm{X}$ lens between the spinning disk unit and the camera. The latter system was equipped with $491 \mathrm{~nm}$ and $561 \mathrm{~nm}$ solid-state lasers. Data acquisition was performed using Metamorph software (Molecular Devices, Meta Imaging Series 7.7). Typical image acquisition settings were $1 \mathrm{X}$ gain, 300 EM gain, with exposure times of 100 ms to 1000 ms for YFP-TUA5 and 500 ms to $2000 \mathrm{~ms}$ for propidium iodide. For imaging, seedlings were transferred to a cover slip (thickness \#1.5) and covered by placing a $1 \mathrm{~mm}$ thick $1 \%$ agarose $(\mathrm{w} / \mathrm{v})$ pad on top of the seedling as described by Lindeboom et al. (2013) ${ }^{44}$ and adding approximately $10 \mu \mathrm{L}$ of sterile water under the agarose pad. Images were processed using Carl Zeiss AIM Image Browser or Image J software ${ }^{73}$. 


\section{Cell wall isolation and composition analysis}

Seven-day-old seedlings grown in liquid cultures were harvested and air dried overnight in a $60^{\circ} \mathrm{C}$ oven. The dry material was then frozen in liquid nitrogen and homogenized to a fine powder using metal balls and an oscillating mill ( 1 minute at $25 \mathrm{~Hz}$ ) from Retsch (Haan, Germany). Cell wall powder was washed with pure ethanol and centrifuged at 13,000 rpm for $10 \mathrm{~min}$. Subsequently, the pellet was resuspended in a 1:1 methanol:chloroform mixture and centrifuged again at $13,000 \mathrm{rpm}$ for $10 \mathrm{~min}$. Lastly, the pellet was resuspended in pure acetone and centrifuged again at $13,000 \mathrm{rpm}$ for $10 \mathrm{~min}$. After that, the cell wall pellet was air dried overnight. The dry, insoluble part of the cell wall material was weighed to approximately $700-800 \mu \mathrm{g}$ per $2 \mathrm{~mL}$ screw-cap tube. Subsequently, $250 \mu \mathrm{L}$ of $2 \mathrm{M}$ trifluoracidic acid was added to the tubes and incubated for $1 \mathrm{~h}$ at $121{ }^{\circ} \mathrm{C}$. Afterwards, the tubes were supplemented with $300 \mu \mathrm{L}$ of 2-Propanol and left for evaporation under a steady air flow at $40{ }^{\circ} \mathrm{C}$. This step was repeated two times before the tubes were supplemented with $300 \mu$ distilled water, thoroughly vortexed and centrifuged at 13,000 rpm for $15 \mathrm{~min}$. The pellet was used to determine the amount of crystalline cellulose using the Updegraff method ${ }^{74}$.

\section{Results}

\section{Ectopic induction of VND7 expression causes Arabidopsis cells to transdifferentiate into vessel element-like cells with secondary cell wall thickenings.}

Firstly, we optimised the induction efficiency of the Arabidopsis lines with inducible VND7 expression ${ }^{51}$, which we will refer to as VND7-seedlings. To determine the optimal dexamethasone (DEX) concentration for VND7 induction a concentration range of $\operatorname{DEX}(1,5,10,50,100 \mu \mathrm{M})$ was applied to 5-day old VND7-seedlings (Table 2.1). At $10 \mu \mathrm{M}$ DEX the ectopic transdifferentiation of epidermal and cortex cells into vessel-like elements had an optimal efficiency with $31.6 \%$ of the plants showing transdifferentiated cells in the hypocotyl. We observed cells with secondary cell wall thickenings in roots, hypocotyl, petiole and the cotyledon after induction of VND7 expression. Secondary cell wall thickenings were consistently present in the hypocotyl, petiole and cotyledon while the observation of transdifferentiated cells in roots was difficult to reproduce. Root cells were therefore less suitable for further experiments. To assess the validity of ectopic VND7-induced transdifferentiation as a model system for endogenous protoxylem vessel element differentiation, we compared the patterns of secondary cell wall thickenings in endogenous protoxylem vessel elements with those observed in transdifferentiated epidermal cells. In endogenous vessel elements it is difficult to 
find the cell-cell boundary of two consecutive cells (Fig. 2.1A). The reason for this might be that the helical pattern of secondary cell wall thickenings is continuously over multiple cells and that cross walls are degraded in the process of vessel development. In transdifferentiating plants, multiple neighbouring epidermal cells do transdifferentiate, but the cross wall between two neighbouring cells remains intact (Fig. 2.1B). Unlike in endogenous protoxylem vessel elements, at the top and the bottom of transdifferentiated hypocotyl epidermal and cortical cells, the pattern of secondary cell wall thickenings is deviating from the regular striated pattern observed in the middle of the hypocotyl cell. In cotyledon cells deviations from the striated pattern are apparent at the lobes of the pavement cells (Fig. 2.1C).

A

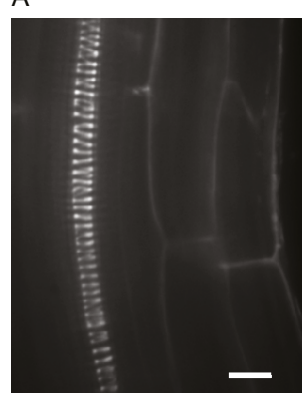

B

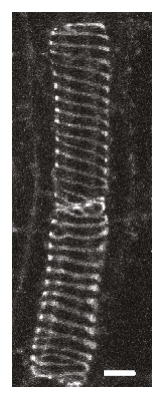

C

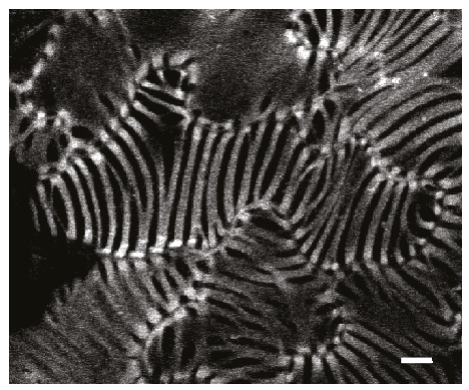

$\mathrm{D}$

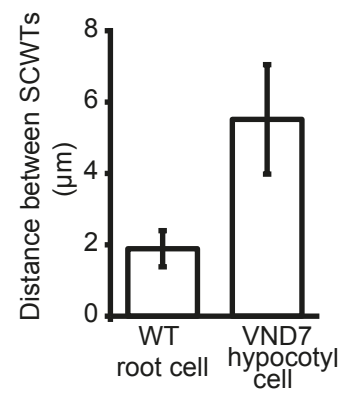

Figure 2.1 | Endogenous secondary cell wall thickenings in wild type roots and ectopically transdifferentiated secondary cell wall thickenings in induced VND7 hypocotyl and cotyledon cells of VND7-seedlings. The secondary cell wall thickenings are stained with $10 \mu \mathrm{M}$ propidium iodide and are visible as white bands in a striated pattern. (A) Endogenous vessel elements of a wild type root. (B) Ectopically induced vessel element-like secondary cell wall thickenings in the hypocotyl of a VND7-seedling. (C) Ectopically induced vessel element-like secondary cell wall thickenings in the cotyledon of a VND7-seedling. All scale bars $=10 \mu \mathrm{m}$. (D) The distance between two consecutive secondary cell wall thickenings (SCWTs) compared between wild type (WT) root cells and VND7-induced hypocotyl cells differs by $\sim 3.5 \mu \mathrm{m} \pm$ s.d. (WT: $N=2$ plants, 1507 measurements, VND7: 4 plants, 402 measurements).

Thus, although cotyledon pavement cells may be used for characterization of the effect of cell shape, these cells are not suitable to reproduce the regular patterning that we observed in protoxylem vessel elements. Hypocotyl cells differ in cell size from protoxylem vessel elements and pattern dimensions differ. The distance between consecutive secondary cell wall thickenings in wild-type root cells was $1.89 \mu \mathrm{m} \pm 0.5 \mu \mathrm{m}$ whereas the distance was $5.52 \mu \mathrm{m} \pm 1.5 \mu \mathrm{m}$ in VND7 transdifferentiated hypocotyl cells into protoxylem vessel elements (Fig. 2.1D). Nonetheless, they have a regular shape and display an even patterning of secondary cell wall thickenings (Fig. 2.1AB). Therefore, we used hypocotyl epidermal cells for further experiments. 
Table 2.1 | VND-induction in 5-days old VND7-seedlings is optimal at $10 \mu \mathrm{M}$ DEX in the hypocotyl with an induction rate of $31.6 \%$.

\begin{tabular}{llll}
\hline DEX & \# plants & $\begin{array}{l}\text { \# plants with VND-induction } \\
\text { in hypocotyl }\end{array}$ & $\begin{array}{l}\text { \% plants with VND-induction } \\
\text { in hypocotyl }\end{array}$ \\
\hline $1 \mu \mathrm{M}$ & 18 & 4 & $22.2 \%$ \\
$5 \mu \mathrm{M}$ & 12 & 2 & $16.7 \%$ \\
$10 \mu \mathrm{M}$ & 19 & 6 & $31.6 \%$ \\
$50 \mu \mathrm{M}$ & 18 & 4 & $22.2 \%$ \\
$100 \mu \mathrm{M}$ & 16 & 2 & $12.5 \%$ \\
\hline
\end{tabular}

\section{Cortical microtubules are organized into bands separated by gaps that lack microtubules between the bands during protoxylem formation}

To study the microtubule organization during xylem formation we imaged fluorescently labelled microtubules (YFP:TUA5) over time in a hypocotyl cell of a VND7-induced seedling (Fig. 2.2). Within 4 hours after induction of VND7 expression, the microtubule array reorganized from an initial interphase organization in which microtubules were distributed over the cortex of the cell into a banded pattern in which most microtubules were located within microtubule bands. The microtubule pattern observed $16 \mathrm{~h}$ after induction (Fig. 2.2; $16 \mathrm{~h}$ ai) was similar to the secondary cell wall thickening pattern as observed in the end stage of a transdifferentiated hypocotyl cell of a VND7-induced seedling (Fig. 2.1B). The microtubules in the microtubule bands are dynamic and most likely microtubule bundles are present, but not all microtubule are incorporated in a bundle. Single microtubules can be observed growing and shrinking in the microtubule band area.

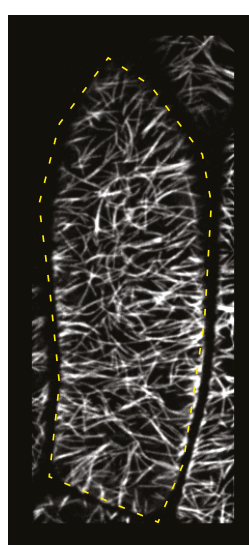

$12 \mathrm{~h}$ ai

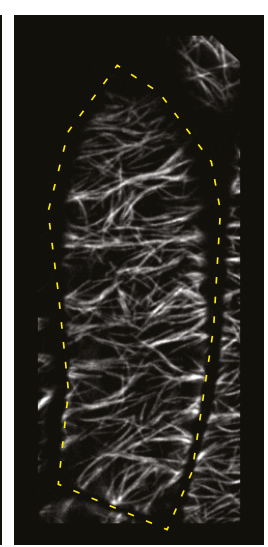

13h ai

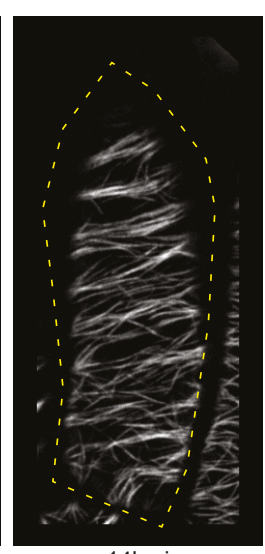

$14 \mathrm{~h}$ ai

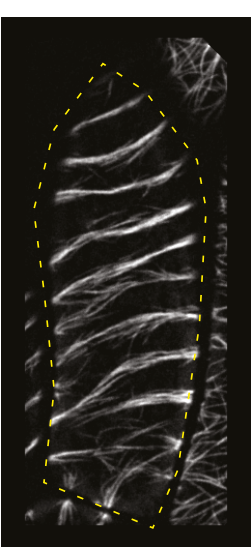

$15 \mathrm{~h}$ ai

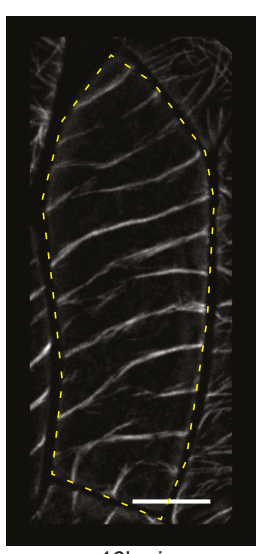

16h ai

Figure 2.2 | After induction of VND7 expression the cortical microtubule array reorients from an interphase organization to a banded pattern. The microtubule array of a hypocotyl cell was followed from $12 \mathrm{~h}$ to $16 \mathrm{~h}$ after VND7-induction (ai). The microtubules reorganize into bands perpendicular to the long axis of the cell. The gap between the microtubule bands gradually becomes devoid of microtubules over time. The images are single focal planes of the cortex of an epidermal hypocotyl cell. Scale bar $=10 \mu \mathrm{m}$. 


\section{Microtubule depolymerisation impairs secondary cell wall thickening deposition and patterning}

To address the impact of microtubules on localized deposition of cell wall thickenings, we applied $10 \mu \mathrm{M}$ of the microtubule depolymerising drug oryzalin to the growth medium at different time points before and afterVND7-induction. These oryzalin depolymerisation experiments were performed in plants with inducible VND7 expression without a microtubule marker since crosses were not available yet at the time of the experiments. Therefore we applied oryzalin to Arabidopsis seedlings with fluorescently labelled microtubules (YFP:TUA5). Within $2 \mathrm{~h}$ after oryzalin application, no fluorescently labelled microtubules could be detected in hypocotyl cells of YFP:TUA5 plants. Application of oryzalin prior to, or during VND7-induction resulted in a significant decrease in cells that deposited secondary cell wall thickenings (Fig. 2.3A). The presence of secondary cell wall thickenings was scored and data was pooled to discriminate between no transdifferentiation ( 0 cells), low transdifferentiation ( $<10$ cells) and high transdifferentiation efficiency (>10 cells). In $80 \%$ of the control seedlings more than 10 cells with secondary cell wall thickenings were present, whereas in the VND7-seedlings treated with oryzalin $3 \mathrm{~h}$ before and $6 \mathrm{~h}, 12 \mathrm{~h}$, and $24 \mathrm{~h}$ after VND7-induction no plants with more than 10 cells with secondary cell wall thickenings were found. At these time points, we did find hypocotyl cells with secondary cell wall thickenings in induced VND7-seedlings, but always less than 10 cells per plant. We hypothesised that the microtubule pattern influences the secondary cell wall thickening pattern early in the transdifferentiation process but that as soon as the pattern is established the microtubules become less important. This was confirmed by the observation that oryzalin-treatment as late as $48 \mathrm{~h}$ after VND7-induction did not reduce the number of cells with secondary cell wall thickenings and showed similar results as the untreated control. Thus, microtubules are important for secondary cell wall thickening deposition up to $24 \mathrm{~h}$ after VND7-induction. Besides the sharp decrease in cells with secondary cell wall thickenings, we observed cells with disorganized cell wall thickenings in VND7-seedlings treated with oryzalin $6 \mathrm{~h}$ to $24 \mathrm{~h}$ after VND7-induction. Different types of secondary cell wall thickening disruptions were observed (Fig. 2.3D). The observed secondary cell wall thickenings were deposited in small patches or as irregular, intermitted bands. Furthermore, the edges of secondary cell wall thickenings were not consistently straight but had a wavy appearance.

The reduced number of cells with secondary cell wall thickenings after treatment with $10 \mu \mathrm{M}$ oryzalin up to $24 \mathrm{~h}$ after induction of VND7 expression could mean that microtubules are required for deposition of secondary cell wall material or that microtubules are needed for patterned deposition of cell wall material. To discriminate between these two hypotheses, we induced 5 day old dark-grown 
VND7-seedlings in presence of $10 \mu \mathrm{M}$ oryzalin and measured the amount of crystalline cellulose after $48 \mathrm{~h}$ using the Updegraff method ${ }^{74}$. We found that VND7 expressing seedlings showed a significantly higher amount of cellulose deposition when compared to non-treated control seedlings, showing that this assay is sufficiently sensitive to detect secondary cell wall deposition. Of the induced VND7-seedlings, oryzalin-treated seedlings did show equal cellulose levels as non-treated seedlings (Fig. 2.3B). This result indicates that microtubules are not necessary for the deposition of secondary cell wall material. However, they are required for correct patterning of secondary cell wall thickenings.

A

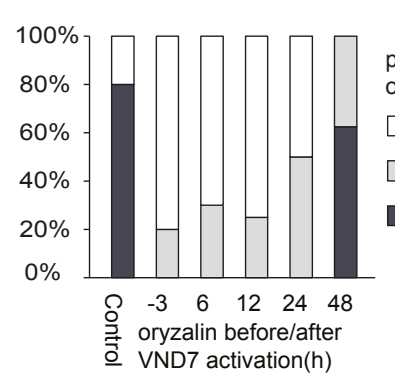

C

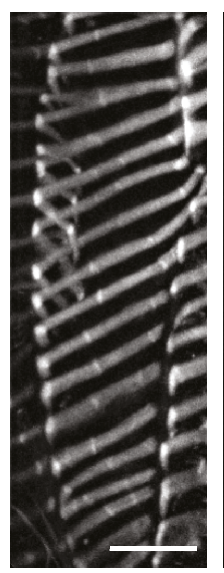

D

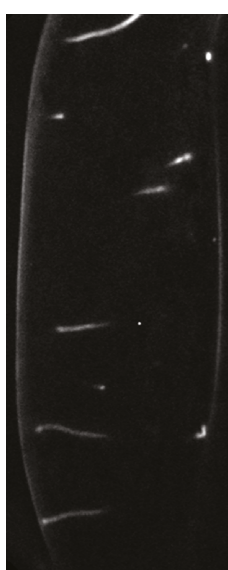

B

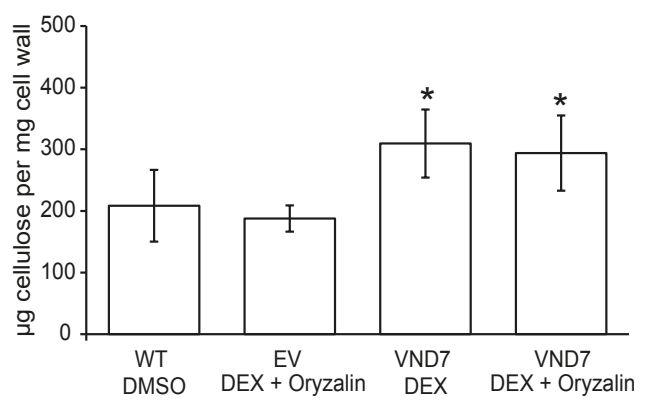

DMSO DEX + Oryzalin DEX DEX + Oryzalin

Figure 2.3 | Oryzalin-treatment causes fewer and misshaped secondary cell wall thickenings but the cellulose levels are unaffected. (A) VND7 is induced in 3 day old VND7-seedlings without oryzalin (control) or with $10 \mu \mathrm{M}$ oryzalin 3h before VND7-induction and 6, 12, 24 or $48 \mathrm{~h}$ after VND7-induction. The presence of secondary cell wall thickenings (SCWTs) is assessed in hypocotyl cells 2 days after oryzalin treatment ( $N \geq 5$ seedlings per treatment). (B) Quantification of the amount of cellulose in cell wall extracts of 7 day old wild-type (WT), empty vector (EV), and VND7-seedlings $48 \mathrm{~h}$ after treatment. ${ }^{*} p<0.05$, Welch's unpaired t-test, numbers are averages from 3 biological replicates with 3 technical replicates each. (C) A VND7-induced hypocotyl cell without oryzalin treatment shows a regular secondary cell wall thickening pattern. (D) VND7induced cells treated with oryzalin $8 \mathrm{~h}$ after induction show abnormal patterned secondary cell wall thickenings. The secondary cell wall thickenings are discontinuous and irregular. The secondary cell wall thickenings are imaged 5 days after VND7-induction and stained with propidium iodide. Scale bar $=10 \mu \mathrm{m}$. 


\section{Taxol-treatment accelerates microtubule bands and secondary cell wall thickening formation and increases the amount of cellulose deposition after VND7-induction}

In Figure 2.3 we showed that treatment with the microtubule depolymerising drug oryzalin affects the appearance of secondary cell wall thickenings. Next we asked if stabilization of microtubules alters the patterned deposition of secondary cell wall thickenings as well. To test this, we induced 5 day old dark-grown VND7seedlings in presence of $20 \mu \mathrm{M}$ taxol and measured the amount of crystalline cellulose after $48 \mathrm{~h}$ using the Updegraff method ${ }^{74}$ (Fig. 2.4C). We found that the taxol-treated seedlings produced significantly more cellulose (381 $\pm 40 \mu \mathrm{g}$ per mg cell wall material) compared to non-treated induced VDN7-seedlings $(309 \pm 55 \mu \mathrm{g})$. What causes the increased cellulose content in cell walls of taxoltreated VND7-seedlings? Possible explanations may be that cell wall deposition starts earlier and/or that cell wall deposition proceeds faster. We compared the speed of progression of the VND7 developmental program between non-treated and taxol-treated seedlings by visualizing the cortical microtubule arrays and secondary cell wall thickenings of 3 day old seedlings $12 \mathrm{~h}$ after VND7-induction with or without taxol-treatment.

We observed different states of progression. To quantify this, we categorized the microtubule array and the secondary cell wall thickenings into three different progression states: early, mid and late stages, similar to Watanabe et al. $(2015)^{52}$ (Fig. 2.4A). We found that $60 \%$ of the hypocotyl cells showed microtubule bands in induced VND7-seedlings. In contrast, for taxol-treated seedlings $29 \%$ of the hypocotyl cells showed microtubule bands (Fig. 2.4B). This finding indicates that microtubule bands form earlier when taxol is present. Furthermore, we also observed that secondary cell wall thickenings were present in almost $80 \%$ of the hypocotyl cells of taxol-treated seedlings whereas only about $20 \%$ of the hypocotyl cells in induced control seedlings showed secondary cell wall thickenings (Fig. 2.4B). This observation indicates that secondary cell wall thickening deposition occurs earlier in the presence of taxol. The finding that taxol not only speeds up microtubule rearrangements but also causes an earlier onset of cellulose deposition hints toward a feedback mechanism between the status of the microtubule array and the start of the secondary wall synthesis. Interestingly, we found that the premature onset of cellulose deposition lead to enhanced levels of cellulose being deposited after 48 hours of induction (Fig. 2.4C). However, the overall order and regularity of the deposited secondary cell wall thickenings was defective leading to more bifurcated and curved secondary cell wall thickenings that were also spaced wider apart (Fig. S2.1). Thus, the structural support provided by the secondary cell wall thickenings of protoxylem vessel elements produced under these circumstances is likely to be reduced. 

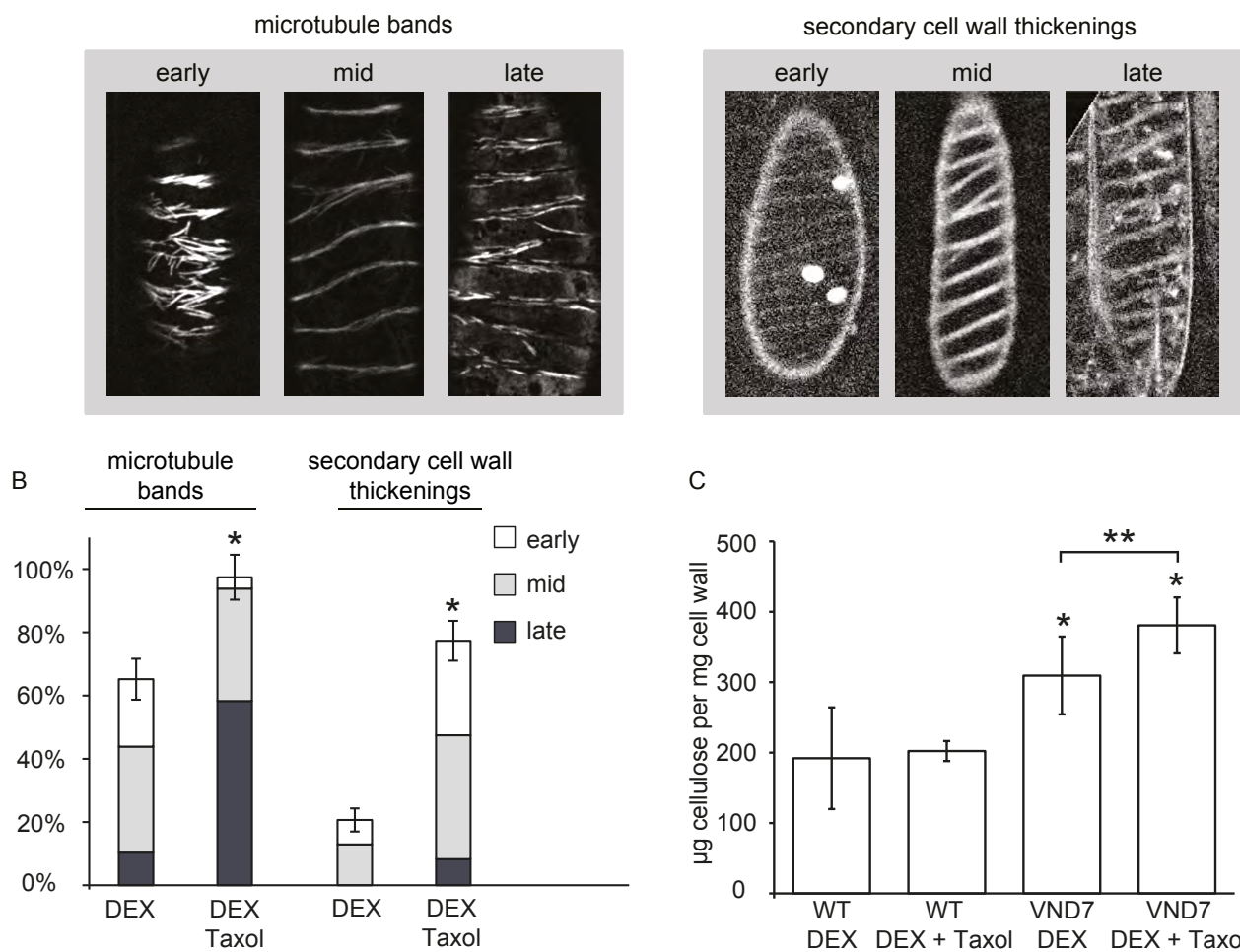

C

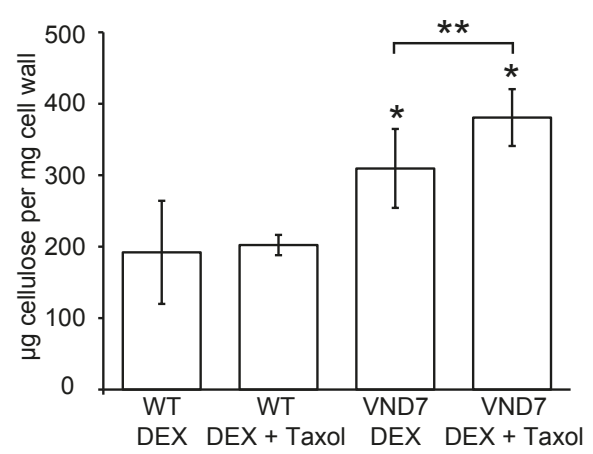

Figure 2.4 | Microtubule stabilization by taxol results in an accelerated formation of microtubule bands and secondary cell wall thickenings. (A) The microtubule array and the secondary cell wall thickenings in induced VND7-seedlings were categorized into three different states: early, mid and late. Note that microtubule bands form before secondary cell wall thickenings occur. Thus, a cell can have late microtubule bands with the secondary cell wall still being in the early state. (B) The percentage of hypocotyl cells with microtubule arrays and secondary cell wall thickenings in the early, mid or late state $12 \mathrm{~h}$ after VND7-induction. When seedlings are induced with DEX + taxol the microtubule bands are less in the early state and more in the late state indicating an acceleration of microtubule patterning in the presence of taxol. Significance is tested using Welch's unpaired t-test, $p<0.01$. The difference to $100 \%$ originates from cells whose microtubule array has not started to reorganize and cells which did not show secondary cell wall thickenings yet. (C) Quantification of the amount of cellulose in cell wall extracts of 7 day old wild-type (WT), and VND7-seedlings $48 \mathrm{~h}$ after taxol treatment. Significance is tested using Welch's unpaired t-test, VND7 vs WT-control ${ }^{*} p<0.05$, VND7 treated with DEX + taxol vs VND7 with DEX, ${ }^{* \star} p<$ 0.05 , numbers are averages from 3 biological replicates with 3 technical replicates each.

\section{VND7-induction results in a gradual increase in the number of cells that have undergone programmed cell death}

Treacher elements undergo programmed cell death before they are functional to conduct water flow. To assess the progress of the xylogenesis developmental program we analysed the live-dead ratio at different time points after VND7- 
induction by a fluorescein diacetate (FDA) live-dead assay (Fig. 2.5A). FDA is converted into fluorescein by intracellular esterase activity in living cells, resulting in a selective fluorescent staining of live cells ${ }^{75}$. All cells are viable $24 \mathrm{~h}$ after VND7induction. The first dead cells are observed 36h after induction (Fig. 2.5B). After $48 \mathrm{~h}$ more than half of the observed cells are dead (57\%) and the number of dead cells increases up to $120 \mathrm{~h}$ (Fig. 2.5B). The majority of cells are dead by $72 \mathrm{~h}(91 \%)$. The patterning of secondary cell wall thickenings and their deposition are cell processes occurring when the cell is still alive. These results indicate that further experiments to investigate how secondary cell wall thickenings are patterned during xylem formation should focus on the first 48h after VND7-induction.

A

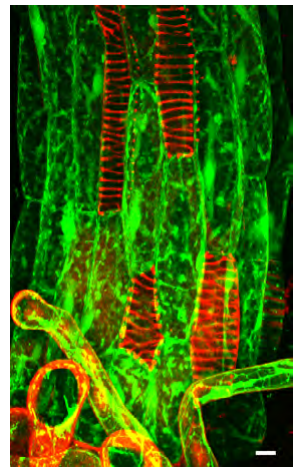

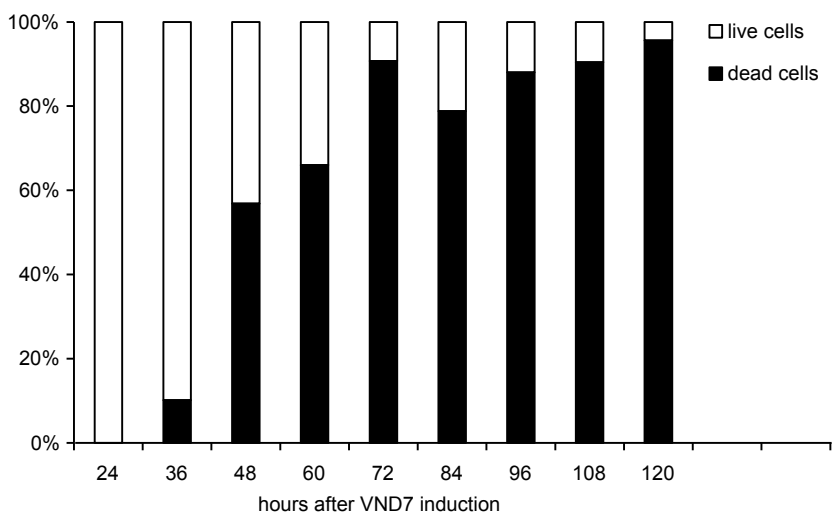

Figure 2.5 | Live-dead analysis of VND7 induced hypocotyl cells using fluorescein diacetate (FDA). (A) Hypocotyl cells of induced VND7-seedling stained with FDA (green) as life-stain and propidium iodide (red) to stain secondary cell wall thickenings. The cells with patterned secondary cell wall thickenings do not show the FDA signal and are dead cells. Scale bar $=10 \mu \mathrm{m}$. (B) Live-dead cell ratios of cells that have entered (alive) or completed (dead) the transdifferentiation process into vessel elements. All cells are alive $24 \mathrm{~h}$ after VND7-induction while from $36 \mathrm{~h}$ up to $120 \mathrm{~h}$ the number of dead cells increases.

\section{Temporal sequence of xylem formation via VND7-induction}

In order to study the transdifferentiation of cells afterVND7-induction it is important to know the temporal sequence of events. The first detected microtubule array reorganizations were observed by spinning disc microscopy 6h after VND7induction. The first deposition of secondary cell wall thickenings was detected $12 \mathrm{~h}$ after VND7-induction. Since the oryzalin treatment did not have an effect on the number of cells with secondary cell wall thickenings when applied 48h after VND7induction, this represents an upper boundary for the microtubule reorganization. From $36 \mathrm{~h}$ onwards programmed cell death is observed representing the end of protoxylem formation transdifferentiation. These temporal marks allow for the creation of a temporal model of cell developmental processes started by 
the VND7-induction (Fig. 2.6). The complete transdifferentiation pathway takes about 2-3 days from DEX application to programmed cell death. The time marks in this temporal model are indications when the majority of cells are in the next stage of transdifferentiation. To investigate the pattern formation of microtubules after VND7-induction the focus should be on the time window between $6 \mathrm{~h}$ and about 40h after VND7-induction. This is the moment with the highest chance to capture the microtubule array transition from an initial array to a patterned array as observed in Figure 2.2.

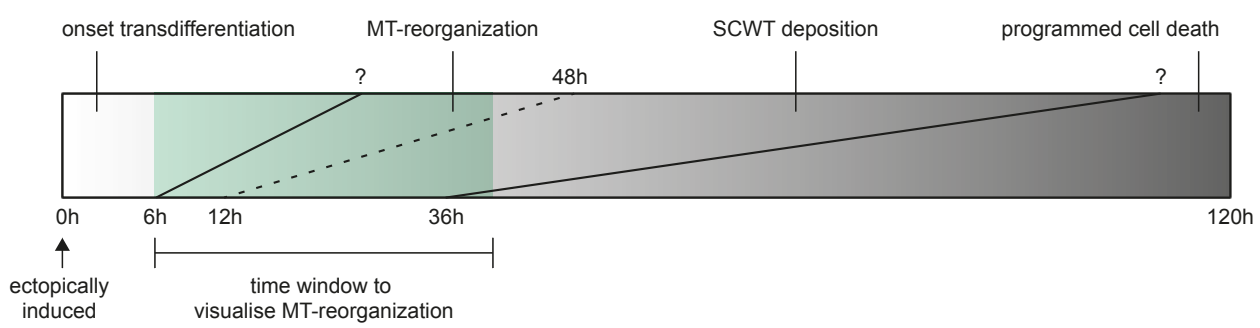

Figure 2.6 | Temporal model of cell developmental processes of the VND7 transdifferentiation program. The VND7-induction is started by the application of DEX at Oh. The onset of transdifferentiation triggers a molecular cascade of developmentally regulated genes ${ }^{45}$. The first detectable change by confocal microscopy is the reorganization of the microtubule array at $6 \mathrm{~h}$ after VND7-induction. The end of the time frame during which DEX induces VND7 expression is unknown. Secondary cell wall thickening (SCWT) deposition is first observed $12 \mathrm{~h}$ after VND7induction. It is unknown how strict the transition is from microtubule (MT) reorganization to secondary cell wall thickening deposition represented by the dashed line. Since the oryzalin treatment does not affect secondary cell wall thickening deposition when applied 48h after VND7induction, this implies that the microtubule reorganization is finished by then. The live-dead assay tells us that the first programmed cell death is occurring $36 \mathrm{~h}$ after VND7-induction and continues up to $120 \mathrm{~h}$. The green zone indicates the time window when to visualize transdifferentiating cells in order to investigate microtubule reorganization after VND7-induction.

\section{Discussion}

In this chapter we determined the time-course of VND7-induced transdifferentiation of hypocotyl cells into protoxylem vessel elements and we have assessed the role of cortical microtubule over time in the deposition of localized cell wall thickenings by treatments with microtubule disrupting and stabilizing drugs. We show that the microtubule array provides cues for the patterning of secondary cell wall thickenings since (1) microtubules are aligned with future secondary cell wall thickening locations early in the developmental program, (2) secondary cell wall deposition continues, but patterning is absent when microtubules are depolymerised and (3) microtubule stabilization accelerates pattern formation and causes earlier deposition of secondary cell wall thickenings. We provide a temporal model that specifies the time window for investigation of microtubule reorganization in transdifferentiating 
VND7-seedlings from an initial interphase pattern to the patterned microtubule array observed during xylem formation (Fig. 2.2 \& 2.6).

Cells in which ectopic VND7 expression is induced show a drastic change in organization of the microtubule array. Microtubules cluster together in microtubule bands and are spaced by gaps devoid of microtubules. To facilitate this reorganization it is likely that microtubule dynamics are locally modulated. The growth and shrinkage velocity, together with the catastrophe and rescue rate determine the length characteristics of microtubules in a system. The observed pattern can emerge if the local microtubule dynamics favour growth in the microtubule band area and opposes growth in the microtubule gap area. Microtubule associated proteins (MAPs) are proteins able to change the microtubule dynamics at the microtubule level.

Transcriptional analysis of VND-induced Arabidopsis revealed two putative MAP kinesins which were upregulated in transdifferentiating cell cultures ${ }^{45}$. In metaxylem differentiation, AtKinesin13A has been shown to reject microtubule growth from future pit regions by depolymerising microtubules ${ }^{54}$. AtKinsesin $13 \mathrm{~A}$ is expressed during protoxylem differentiation but it is not shown that this MAP is active during protoxylem differentiation. The question therefore remains which MAPs are responsible for the reorganization of the array and how the microtubule pattern remains stable over time to function as a template for the cell wall deposition machinery. To answer this question, our study should be followed up by detailed studies into the microtubule dynamics during xylem formation.

We showed that by stabilizing or disrupting the microtubule array we can modify the deposition of secondary cell wall thickenings in protoxylem vessel formation. Stabilization of microtubules in taxol-treated VND7-seedlings results in an earlier onset of microtubule patterning and deposition of cell wall thickenings (Fig. 2.4B). A similar observation has been made in Zinnia elegans cell cultures, that display increased xylem transdifferentiation speeds when exposed to taxol ${ }^{47}$. We show that this increased transdifferentiation speed is due to an earlier onset of patterning of the microtubule array. The earlier initiation of xylem formation by taxol suggests that microtubule stability is a factor in initiating the onset of secondary cell wall deposition. This implies that microtubule stability is somehow perceived by the secondary cell wall deposition machinery, possibly by a factor that senses microtubule stability. MAP65 is a putative candidate for such a function. Although ZeMAP65 is upregulated in Zinnia elegans cell cultures transdifferentiating into xylem elements ${ }^{46}$, in Arabidopsis up-regulation of the nine AtMAP65s could not be specifically linked to xylem element development ${ }^{49}$. MAP65 bundles antiparallel microtubules but does not stabilize microtubules against depolymerisation or otherwise change the microtubule dynamics ${ }^{76}$. Thus, only stable microtubules are decorated by MAP65. 
In addition, MAP65 is suggested to be able to recruit other factors (e.g. vesicles) to antiparallel microtubule overlaps in the cytokinetic phragmoplast ${ }^{77}$. As such, MAP65 may recruit factors for cell wall deposition specifically to stable microtubules ${ }^{76}$.

Induced VND7-seedlings transdifferentiating in the presence of the microtubule stabilizing taxol have a higher cellulose content compared to untreated transdifferentiating VND7-seedlings (Fig. 2.4). It remains to be determined if the total amount of cell wall has increased or the ratio between cellulose and other cell wall components is modified. A more detailed characterization of cell wall components of taxol-treated VND7-seedlings should be able to provide the information to answer this question. Furthermore, taxol affects secondary cell wall thickening branching and positioning in induced VND7-seedlings (Fig S2.1). As discussed above, if microtubule stability is a factor for recruiting cell wall deposition machinery, taxol treatment may accelerate and increase the amount of recruitment due to its microtubule stabilizing activity.

Destabilization of microtubules by oryzalin results in fewer cells depositing secondary cell wall thickenings. In addition, an increased number of cells with irregularly patterned secondary cell wall thickenings was found compared to control cells if seedlings were treated with oryzalin 6-24h after VND7-induction. The cellulose content is not decreased in the oryzalin-treated VND7-seedling. This indicates that in the absence of microtubules secondary cell wall deposition continues. Thus, microtubules are not necessary for secondary cell wall deposition but they are required for focussed cell wall deposition to form secondary cell wall thickenings. It is known that cellulose synthase movement is coordinated by cortical microtubules, but in the absence of microtubules cellulose deposition continues ${ }^{38}$. Without guidance by cortical microtubules it is likely that the cellulose deposition is incorporated evenly in the existing primary cell wall matrix. The intermitted secondary cell wall thickening patterns we observe in the oryzalin-treated seedlings might occur when the microtubule array is partly depolymerised so that the remnants of the microtubule array may still act as a guiding pattern for the secondary cell wall deposition that occurs over time. To confirm this hypothesis it is necessary to repeat the microtubule depolymerising experiments in plants expressing a fluorescent microtubule marker.

In conclusion, we have shown that the microtubule array is crucial as a template for spatial patterning of cell wall deposition in transdifferentiating protoxylem vessel elements. Treatments with the microtubule stabilizing drug taxol suggest that microtubule stability serves as a switch towards deposition of secondary cell wall thickenings since the onset of secondary cell wall thickening deposition is accelerated. These results validate the importance of microtubule organization during xylogenesis and pave the way for a more in-depth analysis of the microtubule array reorganization observed during xylem formation. 


\section{Supplementary Figure}

A

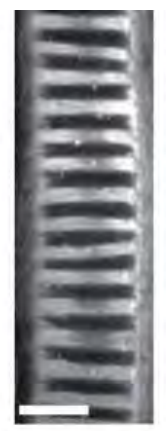

C

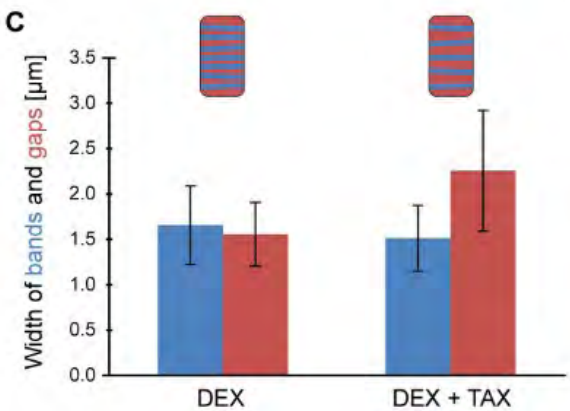

B

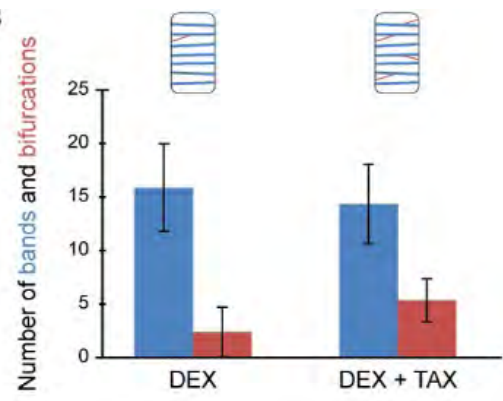

D

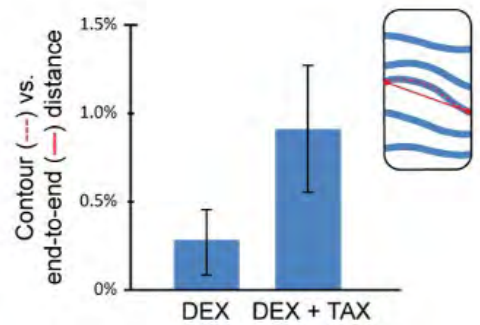

Supplementary Figure S2.1 | The presence of taxol during protoxylem transdifferentiation causes defects in branching, shape and spacing of secondary cell wall thickenings. (A) Imaging of DirectRed23-stained secondary cell wall thickenings was performed on 7-day old VND7seedlings being induced and taxol-treated for $48 \mathrm{~h}$. Arrows indicate bifurcations of secondary cell wall thickenings. Scale bar $=10 \mu \mathrm{m}$. (B) Taxol causes more bifurcations per secondary cell wall thickening $(0.38 \pm 0.14$, mean \pm s.d., $N=11$ cells, $p \leq 0.005$, Welch's unpaired t-test) compared to non-treated induced cells $(0.14 \pm 0.12, N=10$ cells). (C) Taxol caused wider spacing of secondary cell wall thickenings ( $p \leq 0.05$, Welch's unpaired t-test). (D) Taxol caused secondary cell wall thickenings to deviate from a straight line $(0.91 \% \pm 0.36 \%, N=11$ cells, $p \leq 0.005$, Welch's unpaired t-test) compared to non-treated induced cells $(0.28 \% \pm 0.19 \%$, mean \pm s.d., $\mathrm{N}=10$ cells). 



\section{Chapter

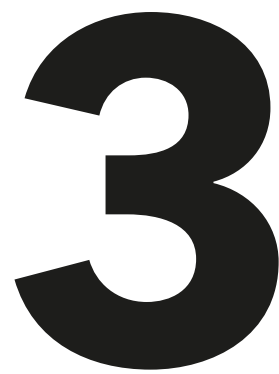

\section{Local destabilization drives}

cortical microtubule rearrangement during protoxylem formation

Kris van 't Klooster*, René Schneider*,

Jasper van der Gucht, Marcel Janson,

Staffan Persson, Tijs Ketelaar

*shared first co-author 


\section{Introduction}

Interphase plant cells have a network of dispersed cortical microtubules located at the cell cortex where they undergo centrosome-independent nucleation, dynamic instability and treadmilling ${ }^{41}$. During growth, the microtubule array is dictating the deposition of the primary cell walls, in particular of the biopolymer cellulose, into evenly dispersed layers. Cellulose is synthesised by large membrane-spanning cellulose synthase (CesA) complexes ${ }^{42}$, which are inserted in close proximity to, and move along cortical microtubules ${ }^{38-40,52}$. The cortical microtubule array thus provides a local bias for where cellulose deposition is required.

When cell growth has ceased, the cell wall of some cell types is reinforced by an additional layer of cell wall, which is referred to as the secondary wall. One function of the secondary wall is to provide structural support to vascular tissue and to plant stature. A prime example of patterned secondary walls is the xylem tissue, which facilitates evaporation-driven transport of water and nutrients from the plant root to shoot. During xylem differentiation patterned secondary wall thickenings are deposited, cross walls are perforated and cells undergo programmed cell death, resulting in inter-connected tubular structures. The secondary walls of protoxylem vessel elements, i.e. initial vessel elements differentiating in elongating young plant tissues, consist of periodically spaced bands and spirals enabling cells to stretch with the surrounding tissue as plant tissues elongates, even after programmed cell death. During protoxylem differentiation cortical microtubule arrays are located in similar patterns as the secondary cell wall patterns typical for the protoxylem ${ }^{78}$ (chapter 2). In metaxylem, i.e. vessel elements differentiating after tissue growth has ceased, a ROP-based reaction-diffusion mechanism, resulting in localized activation of a microtubule catastrophe inducing machinery has been proposed to be responsible for microtubule patterning ${ }^{53,54}$. However, the principles by which microtubules are reorganized during the transition from primary to secondary wall deposition in protoxylem remain largely elusive.

Protoxylem differentiation occurs non-simultaneously in planta with cells typically differentiating in a sequential manner, in tissue buried underneath other cell layers. Thus, using these cells to image processes related to secondary wall synthesis is cumbersome. However, a recent inducible transcription factor-based system provides an elegant approach to trigger protoxylem formation in cells that typically do not make secondary walls ${ }^{51}$. Here, the protoxylem inducing master regulator VASCULAR NAC-DOMAIN (VND)7 is under control of a chemically inducible glucocorticoid-receptor, and causes ectopic protoxylem differentiation upon induction ${ }^{51}$. Recently, Watanabe et al. used VND7-inducible Arabidopsis seedlings expressing YFP-labelled CesA7, a specific CesA for secondary cell wall production, to visualize the behaviour of cellulose synthase complexes 
during secondary wall synthesis ${ }^{52}$. Altogether, the VND7-driven secondary wall program offers great opportunities to study microtubule rearrangements during protoxylem differentiation. By performing long-term live-cell imaging of individual microtubules after ectopic induction of VND7 expression in hypocotyl epidermal cells, we show that the periodic banded patterns of microtubules are underpinned by active microtubule depolymerisation in the gap regions between the bands.

\section{Materials and Methods}

\section{Plant material and growth conditions}

Arabidopsis (Arabidopsis thaliana, Columbia Col-0 ecotype) expressing 35S promoter-driven VND7-VP16-GR and the 35S promoter-driven empty vector control line VP16-GR were crossed with Arabidopsis expressing 35S promoter-driven YFP-TUA5 ${ }^{53}$. The F3 progeny was used for all experiments. Seeds were surfacesterilized by application of $1 \%$ bleach (Sodium hypo-chloride) and $0.1 \%$ Tween 20 for 10 minutes followed by thorough washing with sterile water, then stratified for a minimum of two days at $4^{\circ} \mathrm{C}$ in the dark. Seeds were grown either on Hoagland's modified basal salt mixture (Phyto Technology Laboratories, Kansas, USA, 1 \% agarose) or on half-strength Murashige and Skoog medium (MS, Sigma-Aldrich, $0.68 \%$ agarose) supplemented with $1 \%(\mathrm{w} / \mathrm{v})$ sucrose both at $\mathrm{pH}$ 5.7. The plates were placed vertically in a 16-hour-day phytotron. For cell wall analyses, seeds of wild-type, VND7-seedlings, and the empty vector plants were grown under constant shaking at 100 revolutions per minute in liquid $0.5 \mathrm{MS}$ medium supplemented with $1 \%$ sucrose at $\mathrm{pH} 5.7$ in the dark. After 5 days, the cultures were treated with $10 \mu \mathrm{M}$ dexamethasone (DEX) in water and were collected after another 2 days in the dark.

\section{Induction of xylogenesis}

For long-term microtubule imaging, 3-day old VND7-seedlings were transferred from agarose plates to multi-well plates containing $10 \mu \mathrm{M}$ DEX in water. The multiwell plates were placed on a rotary shaker at 120 revolutions per minute in a $16 \mathrm{~h}$-day phytotron $\left(21^{\circ} \mathrm{C}\right)$. VND7-seedlings were kept in these plates for $6-24 \mathrm{~h}$ after which VND7-seedlings were transferred to a microscope slide for imaging. For Oryzalin experiments, 5 day old VND7-seedlings were transferred from agarose plates to $10 \mu \mathrm{M}$ DEX containing multi-well plates and placed on a rotary shaker (as above). For ' $3 h^{\prime}$ ' hour Oryzalin-treatments the VND7-seedlings were incubated with $10 \mu \mathrm{M}$ Oryzalin 3h prior to DEX-treatment. VND7-seedlings were kept on DEX containing plates until processing or microscopic analysis. 


\section{Cell wall analysis}

Liquid cultures were harvested after 7 days in the dark. Seedlings were stored in $70 \% \mathrm{EtOH}$ for one week. To extract cell wall material, seedlings were first air dried overnight in a $60^{\circ} \mathrm{C}$ oven. The dry material was then frozen in liquid nitrogen and homogenized to a fine powder using metal balls and an oscillating mill ( 1 minute at $25 \mathrm{~Hz}$ ) from Retsch (Haan, Germany). Cell wall powder was washed with pure ethanol and centrifuged at $13.000 \mathrm{rpm}$ for 10 minutes. Subsequently, the pellet was resuspended in a 1:1 methanol:chloroform mixture and centrifuged again at 13.000 rpm for 10 minutes. Lastly, the pellet was re-suspended in pure acetone and centrifuged again at $13.000 \mathrm{rpm}$ for 10 minutes. After that the cell wall pellet was air dried overnight. The dry, insoluble part of the cell wall material was weighed to approximately 700-800 $\mu \mathrm{g}$ per $2 \mathrm{~mL}$ screw-cap tube. Subsequently, $250 \mu \mathrm{L}$ of $2 \mathrm{M}$ trifluoracidic acid was added to the tubes and incubated for $1 \mathrm{~h}$ at $121^{\circ} \mathrm{C}$. Afterwards, the tubes were supplemented with $300 \mu \mathrm{L}$ of 2-Propanol and left for evaporation under a steady air flow at $40{ }^{\circ} \mathrm{C}$. This step was repeated two times before the tubes were supplemented with $300 \mu \mathrm{l}$ distilled water, thoroughly vortexed and centrifuged at $13.000 \mathrm{rpm}$ for 15 minutes. The pellet was further used to determine the amount of crystalline cellulose using the Updegraff method ${ }^{74}$.

\section{Microscopy}

Fluorescence imaging was performed using a spinning disc microscope consisting of a CSU-X1 spinning disk head (Yokogawa) attached to an Eclipse TI inverted microscope (Nikon) equipped with a perfect focus system, an Evolve CCD camera (Photometrics), a Plan Apo 100X/1.4 NA oil immersion objective and a 1.2X lens between the spinning disk unit and the camera. YFP was excited at $491 \mathrm{~nm}$ and propidium iodide and Direct Red 23 at $561 \mathrm{~nm}$, using solid-state lasers. Data acquisition was performed using Metamorph software (Molecular Devices, Meta Imaging Series 7.7). Typical image acquisition settings were $1 \mathrm{X}$ gain, $300 \mathrm{EM}$ gain, with exposure times of $100 \mathrm{~ms}$ to $500 \mathrm{~ms}$ for YFP:TUA5 and $500 \mathrm{~ms}$ to $2000 \mathrm{~ms}$ for propidium iodide and Direct Red 23. For imaging, seedlings were transferred to a cover slip (thickness \#1.5) and covered by placing a $1 \mathrm{~mm}$ thick $1 \%$ agarose (w/v) pad on top of the seedling as described by Lindeboom et al. (2013) ${ }^{44}$. Adding approximately $10 \mu \mathrm{L}$ of sterile water under the agarose pad allowed stable imaging conditions for up to several hours.

\section{Staining of the cell wall}

To stain the cell wall, seedlings were either transferred to $0.5 \mu \mathrm{g} \mathrm{mL}^{-1}$ propidium iodide (Sigma-Aldrich, MO, USA) in water for 10 minutes followed by a 5 minute 
wash step in sterile water or to $0.02 \%$ Direct Red 23 (Sigma-Aldrich, MO, USA) in water for 30 minutes followed by exchange of the medium by sterile water.

\section{Image Analysis}

Images were processed using Fiji software ${ }^{79}$ and publicly available plug-ins such as StackReg, Bleach Correction, Multiple Kymograph, and Walking Average were used. The periodogram analysis was performed using a Matlab-based program, which converted intensity line scans along the growth axis of the cell into power spectral density using the periodogram function. The power spectral density was plotted against the inter-band spacing, derived from taking the inverse of the wavenumber. The peak of the power spectral density was identified as the mean spacing between microtubule bands.

\section{Results}

\section{Microtubule rearrangement in VND7-induced protoxylem formation}

To confirm the effect of xylem transdifferentiation we treated 7-day old wildtype, VND7-seedlings, transgenic Arabidopsis seedlings expressing 35S:VND7VP16-GR, and empty-vector seedlings with $10 \mu \mathrm{M}$ dexamethasone (DEX) ${ }^{51}$. After 72 hours, wild-type and empty-vector seedlings were still growing normally whereas VND7-seedlings had stopped growing, had turned pale and had died (Fig. S3.1A). Induction of VND7-seedlings resulted in transdifferentiated hypocotyl cells with conspicuous periodic and striated cell wall deposition perpendicular to the growth axis (Fig. S3.1C). We measured the amount of cellulose produced in wild-type, VND7-seedlings and empty-vector seedlings and found that after 48 hours VND7-induced seedlings produced about $50 \%$ more cellulose compared to wild-type and empty-vector seedlings (Fig. S3.1D).

To investigate when and how the microtubule array is responding to VND7 induction, we crossed the VND7-seedlings with a YFP-TUA5 reporter line (Materials \& Methods). We imaged cortical microtubules in epidermal hypocotyl cells of 3-day old light-grown seedlings under a confocal spinning disk microscope. At the start of VND7 induction the microtubule array was in a dispersed state, i.e. the microtubules and their orientations were evenly distributed (Fig. 3.1A; left), typically seen in interphase cells producing primary wall cellulose ${ }^{35}$. To monitor the progression of microtubule rearrangements we classified the microtubule array into early, mid, and late stages, similar to Watanabe et al. (2015) $)^{52}$. In early stages of the secondary wall program, the microtubules began to orient perpendicular to the 
growth axis and bands of microtubules became apparent (Fig. 3.1B; 'early'). Bands comprised mainly parallel and anti-parallel dynamic microtubules. In this stage, a subset of the total microtubule population deviated from the main perpendicular orientation of the microtubule array, with some reaching over to neighbouring bands. In mid stages of the secondary wall program, microtubule bands became denser, and fewer microtubules deviated from the main perpendicular orientation (Fig. 3.1B; 'mid'). During late stages of the secondary wall program, we observed splitting and disintegration of microtubule bands characterized by a decrease in microtubule intensity in the band and increased cytoplasmic fluorescence (Fig. 3.1B; 'late'). In this stage, all microtubules were perpendicular to the growth axis. Notably, deposition of cellulose was first detected during the late stages (Fig. S3.1E). When following the progression of the VND7 induced protoxylem transdifferentiation for up to 120 hours we found that cells underwent programmed cell death (chapter 2; Fig. 2.5).
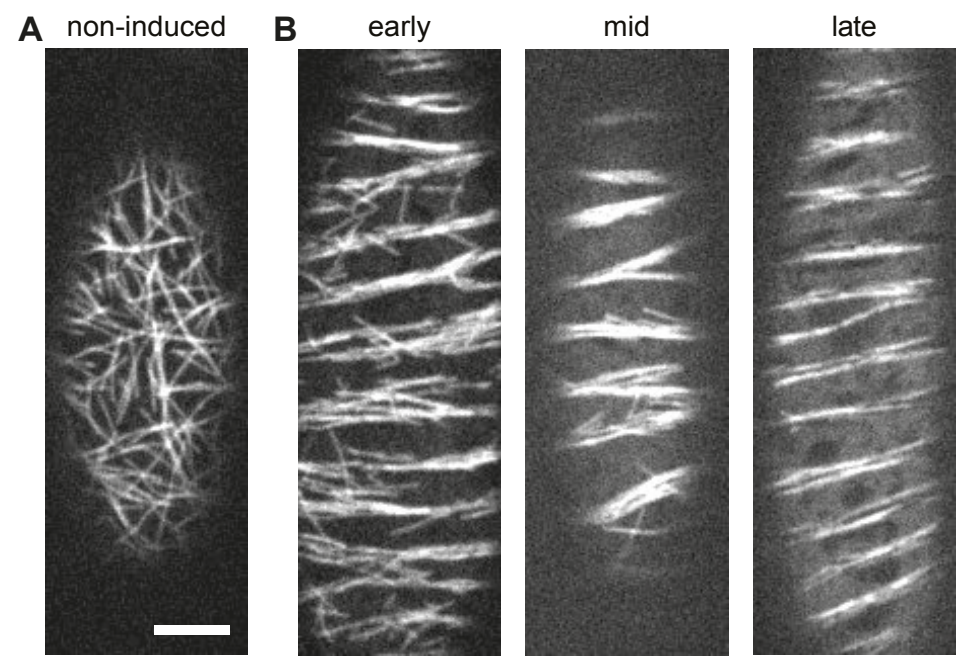

Figure 3.1 | The inducible VND7 system drives ectopic microtubule rearrangements. (A) Representative stages of microtubule rearrangements in non-induced hypocotyl cells and (B) in induced cells during early-, mid-, and late stages of protoxylem transdifferentiation. Scale bar $=5 \mu \mathrm{m}$.

\section{Microtubule rearrangements result in evenly spaced microtubule bands and gaps}

We found that microtubule rearrangement from an initially dispersed pattern into spatially confined bands occurred simultaneously for all microtubules in individual cells (Fig. 3.2A). Microtubule bands had a high microtubule density whereas microtubule gaps, i.e. the areas between bands, showed a reduced density. Most bands were oriented perpendicular to the growth axis of the cell. Only occasionally 
did an individual band show an oblique orientation in mid stages. However, in such cases we observed that the orientation of the band was modulated to become parallel with the neighbouring bands. Furthermore, we observed that microtubule bands can merge (Fig. 3.2AB). When microtubule band formation was completed, bands were equally spaced running in parallel to one another. These observations show that band-band spacing and orientation is tightly controlled and actively modulated during protoxylem differentiation.
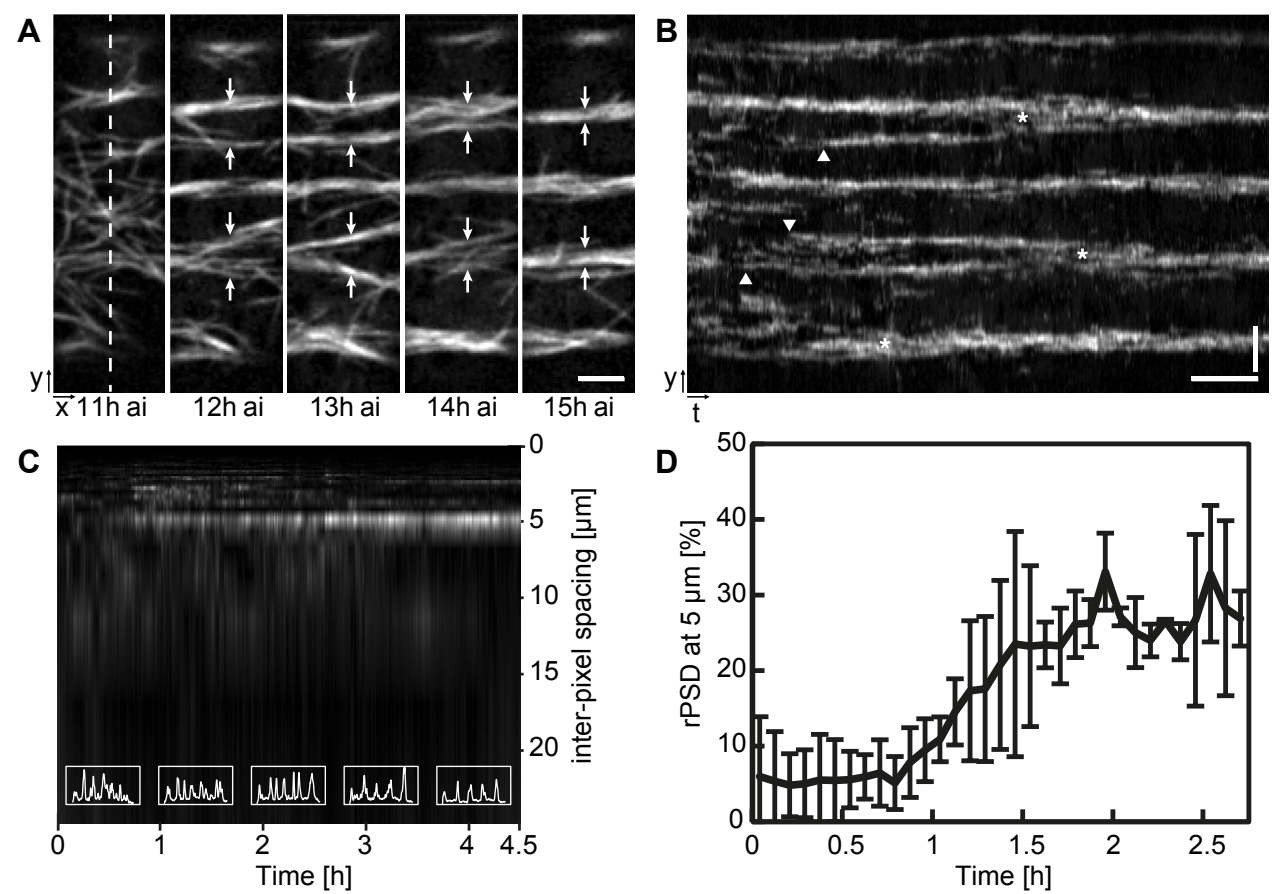

Figure 3.2 | Long time-lapse recordings of induced seedlings reveal the simultaneous formation of microtubule bands and gaps. (A) Time-lapse of microtubule rearrangements in an induced hypocotyl cell. Scale $=3 \mu \mathrm{m}$. Microtubule bands initially emerge in close proximity to each other but merge into one band over time (anti-parallel pair of arrows). (B) Kymograph along the dashed line in (A) reveals the spontaneous emergence (triangles) and gradual merging of microtubule bands (asterisks). Scale bars $=30 \mathrm{~min}$ and $3 \mu \mathrm{m}$. (C) Periodogram of the kymograph in (B) reveals the gradual emergence of a dominant periodic pattern with an average microtubule band spacing of $5.3 \pm 0.5 \mu \mathrm{m}$ (mean \pm s.e., $N=6$ cells, 4 seedlings). (D) Relative power spectral density (rPSD) for an inter-pixel spacing of $5.3 \pm 0.5 \mu \mathrm{m}$ increases rapidly from $5.5 \pm 0.3 \%$ (mean \pm s.e.) to $26.9 \pm 0.5 \%$ within approximately one hour.

To quantify the microtubule array rearrangement we performed a periodogram analysis of the intensity profile along the dashed line in Figure 3.2A. A periodogram provides an estimate for how strong a particular (spatial) frequency is contributing to a fluctuating signal. Therefore, we calculated the relative power spectral density (rPSD) of the intensity profile in Figure 3.2A and plotted rPSD against the various 
inter-pixels spacings present in the profile (Fig. 3.2C). We found, that in the first 30 minutes rPSD was evenly distributed indicating that the intensity profile was dispersed, i.e. the intensity profile revealed equal contributions from all spatial frequencies. Later, rPSD developed a clear peak at an inter-pixel spacing of about $5 \mu \mathrm{m}$. When plotting the temporal development of rPSD for this spacing, we found that the microtubule array rearranges into a periodic banded pattern within one hour (Fig. 3.2D). Taking into account that VND7 induced transdifferentiation is a multiple day process ${ }^{45,51}$ (chapter 2), the periodogram analysis demonstrates that microtubule array reorganization is a rapid process compared to overall transdifferentiation speed.

\section{Microtubule dynamics change over time and differ between microtubule bands and gaps}

We found that cortical microtubules were highly dynamic during the process of microtubule band and gap formation. In particular, we observed that microtubules in gaps often depolymerised. To quantify if microtubule dynamics differ over time and to calculate the mean length of microtubules, we measured the dynamics of microtubules, i.e. growth and shrinkage velocities $\mathrm{V}^{+}$and $\mathrm{V}$, respectively, and catastrophe and rescue rates $R_{\text {cat }}$ and $R_{\text {res }}$ respectively. To achieve this, we acquired 5 -second time-lapses of YFP-labelled microtubules for 5 minutes in intervals of 30 minutes (Fig. 3.3A), and analysed microtubule behaviour in bands and gaps (Fig. 3.3B). Using a walking average image subtraction algorithm we detected growing and shrinking microtubule ends (Fig. 3.3C).

We found that microtubules grew and shrank with a rate of $2.5 \pm 0.5 \mu \mathrm{m} \mathrm{min}{ }^{-1}$ and $4.8 \pm 0.5 \mu \mathrm{m} \mathrm{min}^{-1}$, respectively (mean \pm s.d., $N=14$ time points in one cell, pooled from microtubule bands and gaps at 7 time points). Nevertheless, $\mathrm{V}^{+}$ and $\mathrm{V}$ - did not differ significantly between bands and gaps (Fig. 3.3D; $p=0.75$ and $p=0.13$ for growing and shrinking microtubules, respectively; Welch's unpaired $t$-test). In contrast, $R_{\text {cat }}$ and $R_{\text {res }}$ differed significantly between band and gap regions. Microtubules (MT) in the band underwent catastrophes and rescues at average rates of $0.08 \pm 0.04$ and $0.04 \pm 0.02$ events $\mathrm{min}^{-1} \mathrm{MT}^{-1}$, respectively. Significant higher rates and variations were found in gaps with average $R_{\text {cat }}$ and $R_{\text {res }}$ of $0.22 \pm 0.10$ and $0.10 \pm 0.06$ events min $^{-1} \mathrm{MT}^{-1}$, respectively $(p=0.012$ for catastrophes and $p=0.05$ for rescues; Welch's unpaired t-test; Fig. 3.3 \& S3.2). 
A

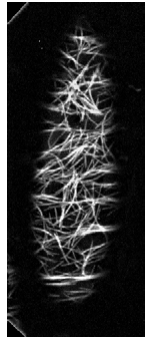

$0: 00 \mathrm{~h}$

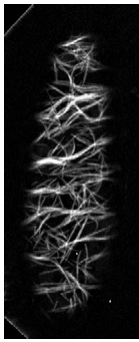

$0: 30 \mathrm{~h}$

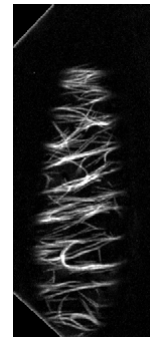

1:00h

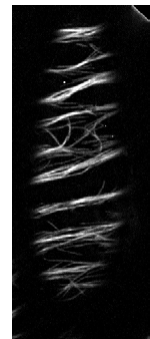

$1: 30 \mathrm{~h}$

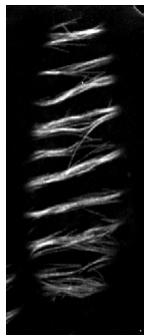

2:00h

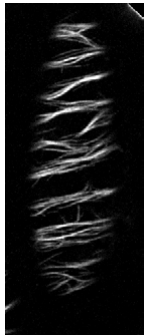

2:30h

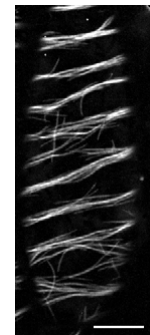

3:00h

B

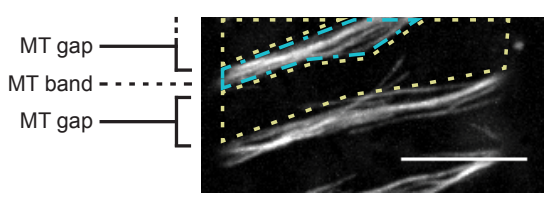

C
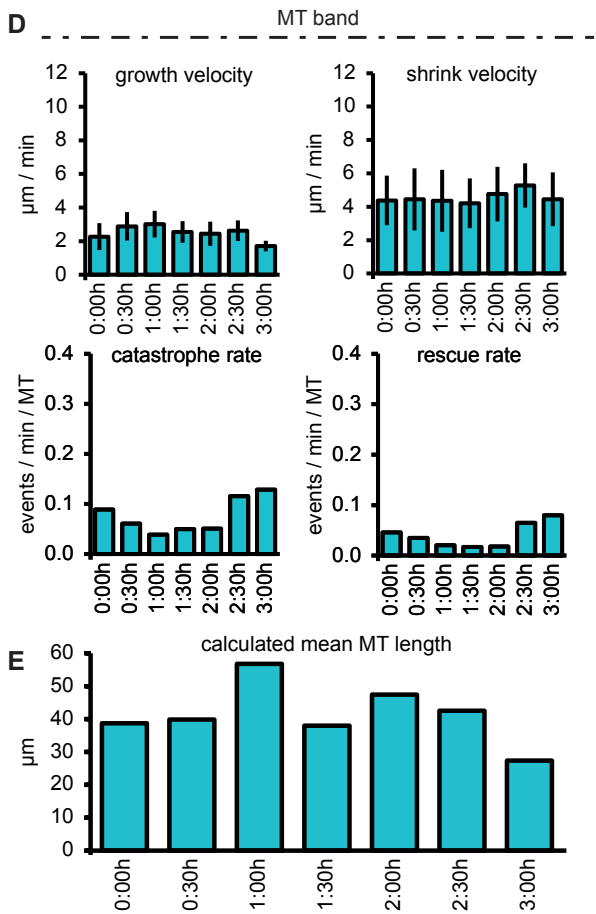

merge MT ends MT ends YFP:TUA5

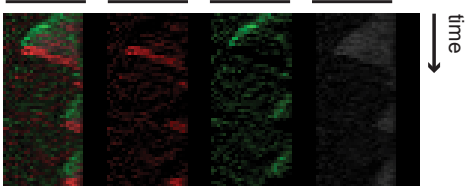

MT gap
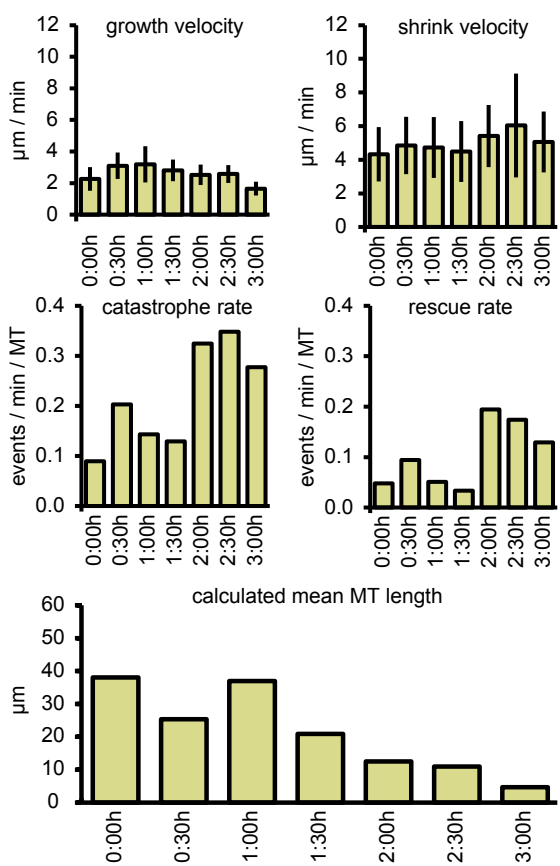

Figure 3.3 | Dynamic microtubules undergo significantly more catastrophes in developing microtubule gap regions. (A) Average images of a three-hour time-lapse of YFP-labelled microtubules in an induced hypocotyl cell. Scale bar $=5 \mu \mathrm{m}$. (B) Definition of microtubule (MT) band and gap regions, respectively. (C) Microtubule (MT) end showing dynamic switching between growing (green) and shrinking (red) states of a microtubule. Transitions from growth to shrinkage were termed 'catastrophe', the inverse transition was termed 'rescue'. (D) Analysis of microtubule dynamics parameters split into microtubule band (cyan) and microtubule gap (yellow) areas. (E) The mean microtubule (MT) length was calculated from the average microtubule dynamics rates of two individual cells for each time point. The microtubule dynamics parameters of the second analysed transdifferentiating hypocotyl cell is depicted in Figure S3.2. 
Growth and shrinkage velocity as well as catastrophe and rescue rates were averaged per time point for the time series. The averages were used to calculate the mean microtubule length $\bar{l}$ for each time point according to the method described by Mulder (2012) $)^{80}$ and in chapter 4 (equation 3). This property does not reflect the actual mean length of the microtubule array but rather represents an extrapolated theoretical length, which the microtubule array would converge to. We note that the first 5 minutes of the time series (Fig. 3.3 \& S3.2) can be consider as suitable start points for analysis of microtubule dynamics because all four microtubule dynamics parameters and $\bar{l}$ were found to be similar for microtubules located in bands and gaps, respectively. We found that the calculated $\bar{l}$ of microtubules located in bands remained around $40 \mu \mathrm{m}$ over time. In contrast, microtubules located in gaps showed a gradual decrease in $\bar{l}$ from around $40 \mu \mathrm{m}$ to $7 \mu \mathrm{m}$. Microtubule gap regions were visible 1.5-2 hours after the first imaged time point of the time series (Fig. 3.3A \& S3.2A). This is captured quantitatively in the periodogram analysis (Fig. 3.2D) and in the increased $R_{c a t}$ in the time series (Fig. 3.3D \& S3.2B) causing the reduced $\bar{l}$ in the microtubule gap region 1.5-2 hours after the first imaged time point of the time series (Fig. 3.2E).

\section{Discussion}

Microtubule patterning drives the deposition of cell wall components, typified in protoxylem differentiation. However, the principles that underpin microtubule reorganization during such differentiation remain largely unknown. We showed that dispersed microtubules rearrange into equally spaced parallel microtubule bands in a non-linear time frame and that this pattern is promoted by spatial differences in catastrophe rates. Our results indicate that unequal microtubule behaviour in bands and gaps over time drives the patterns and impacts on the timing of the deposition of secondary cell wall material.

Patterned secondary wall deposition is best investigated during pit formation, a specific patterned cell wall type of metaxylem vessel elements with small pit regions lacking secondary cell walls. Using VND6, a master regulator of metaxylem development, future pit regions were marked by the active form of the GTPase ROP11, which recruited MICROTUBULE DEPLETION DOMAIN (MIDD)1, a microtubule associated protein (MAP) promoting microtubule disassembly, to the plasma membrane ${ }^{53,55}$. MIDD1 in turn recruited KINESIN-13A, a microtubule motor protein known to depolymerise microtubules ${ }^{54}$. A Turing-like reactiondiffusion mechanism ${ }^{20}$ is suggested to initiate patterning of ROP11 in metaxylem formation, in which ROPGEF4 and ROPGAP3 may respectively act as activator and inhibitor ${ }^{53}$. This mechanism renders ROP11-marked regions to become 
free of microtubules and thus prevents the recruitment of cell wall machinery to these areas. This leads to the perforated secondary cell wall patterns typical for metaxylem vessel elements ${ }^{53,54}$. These studies outlined a potential mechanism for microtubule pattern formation during metaxylem differentiation, but did not investigate how microtubule behaviour changes over time. ROP11-mediated patterning does not seem to function in protoxylem formation, since neither the presence of a constitutive active or dominant negative version of ROP11, nor the absence of ROPGEF4, alter the spacing between secondary cell wall thickenings in the protoxylem ${ }^{53}$. It is currently unclear what the molecular contributors are to protoxylem patterning. Nonetheless, ROP1 and ROP7 are putative candidates since these GTPases are upregulated after VND7 induction ${ }^{81}$.

With our detailed study of microtubule dynamics we show that microtubule behaviour changes spatially and temporally during protoxylem transdifferentiation. In both microtubule gap formation and microtubule pit formation, in the VND7 and the VND6 system respectively, the emergence of a cortical region with a reduced microtubule density is observed. microtubule dynamics in pit regions has been assessed and compared to interphase cells ${ }^{55}$. This allows for some comparison, but note that our data is temporally more detailed and we compare microtubule band and gap regions within the same cell, which guarantees similar experimental conditions between the compared microtubule dynamics parameters. We observed unchanged $\mathrm{V}^{+}$and $\mathrm{V}^{-}$with an increase in $R_{\text {cat }}$ in microtubule gap regions over time, similar as has been observed in microtubule pit formation ${ }^{55}$. This similarity might indicate that microtubule disassembly in microtubule gaps and pits might share a common mechanism. This hypothesis is supported by the presence of AtKinesin$13 \mathrm{~A}$ in protoxylem tissue ${ }^{82}$ and MIDD1 being upregulated after VND7 induction ${ }^{81}$. Contrary to the reduced rescue rate observed in microtubule pits ${ }^{55}$, we find an increase in the rescue rate in microtubule gaps. Therefore, besides shared MAPs, additional MAPs might be involved distinguishing protoxylem and metaxylem patterns during microtubule gap and pit formation. In our work we show that microtubule rearrangement follows three stages in which microtubule dynamics change over time and differ between microtubule bands and gaps. Furthermore, the reorientation and merging of microtubule bands that we observed during early microtubule rearrangements indicates that there is a sensing and spacing mechanism in place between neighbouring microtubule bands and/or gaps. Our results show that for future investigations into microtubule rearrangements during xylem formation, it is important to include spatial and temporal assessment of microtubule dynamics.

The observed change in microtubule dynamics during protoxylem formation generates understanding how the microtubule gap emerges but it is less well 
understood how microtubule bands form. As microtubule gaps are actively created, the results could be that microtubule bands emerge passively. However, the observation that microtubule bands can merge suggests an active regulation of microtubule bands. MAP70-5 may be a factor involved in delimiting microtubule bands, since it localizes specifically to the periphery of bands ${ }^{49}$.

Our results show that the microtubule array functions in positioning and timing of patterned cell wall deposition in the VND7-driven secondary wall program. Patterning of the microtubule array precedes the onset of patterned cell wall deposition. The remodelling of the microtubule bands indicates that the pattern is not exclusively predefined but that as the microtubule bands appear, the pattern is adjusted for optimal spacing of the later formed secondary cell wall thickenings. Our in-depth analysis shows that microtubule dynamics differ between microtubule band and gap regions during protoxylem formation. Local microtubule destabilization causes microtubule gap formation. These results can guide the search for the mechanisms controlling microtubule patterning in protoxylem formation to further our understanding in microtubule patterning in general. 


\section{Supplementary Figures}

A

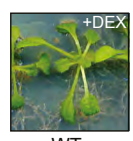

WT

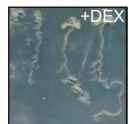

VND7

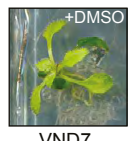

VND7

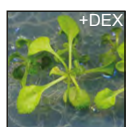

EV
B

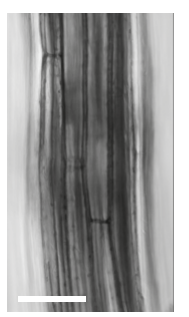

C

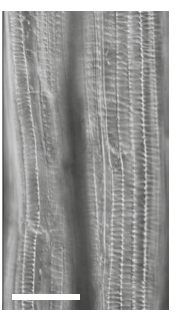

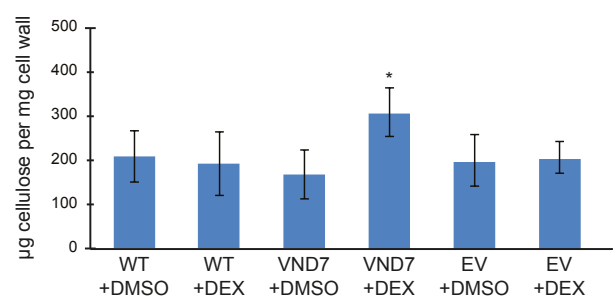

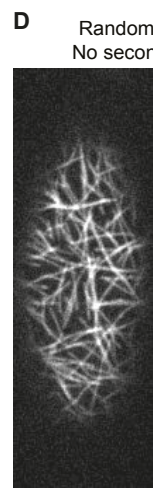

Tubulin

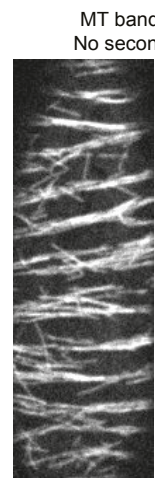

MT bands established No secondary CWT
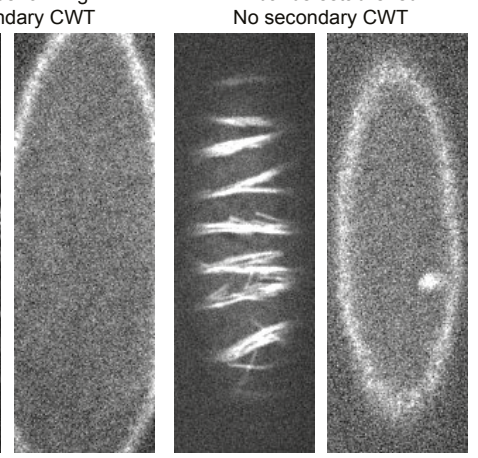

MT bands collapsed Secondary CWT forming
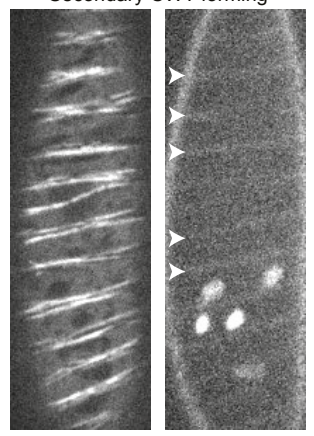

Supplementary Figure S3.1 | The inducible VND7 system drives ectopic cortical microtubule rearrangement and secondary wall cellulose deposition. (A) Images of 10-day old light-grown wild-type, VND7 and empty-vector seedlings treated with $10 \mu \mathrm{M}$ DEX and DMSO, respectively, for 72h. (B,C) DIC images of 10-day old dark-grown hypocotyls of wild-type (B) and VND7-seedlings (C). Scale bar $=100 \mu \mathrm{m}$. (D) Induced VND7-seedlings contain significantly more cellulose in their cell walls ( $309 \pm 55 \mu \mathrm{g}$ per mg cell wall material) compared to wild-type ( $208 \pm 58 \mu \mathrm{g}$ ) and empty-vector seedlings $(199 \pm 59 \mu \mathrm{g})$. mean \pm s.d., $N=3$ biological replicates with 3 technical replicates, ${ }^{*} p \leq 0.05$, Welch's unpaired $t$-test. (E) Representative stages of the secondary wall program: microtubule rearrangements precede the deposition of secondary walls (arrows heads). Scale bar $=5 \mu \mathrm{m}$. 

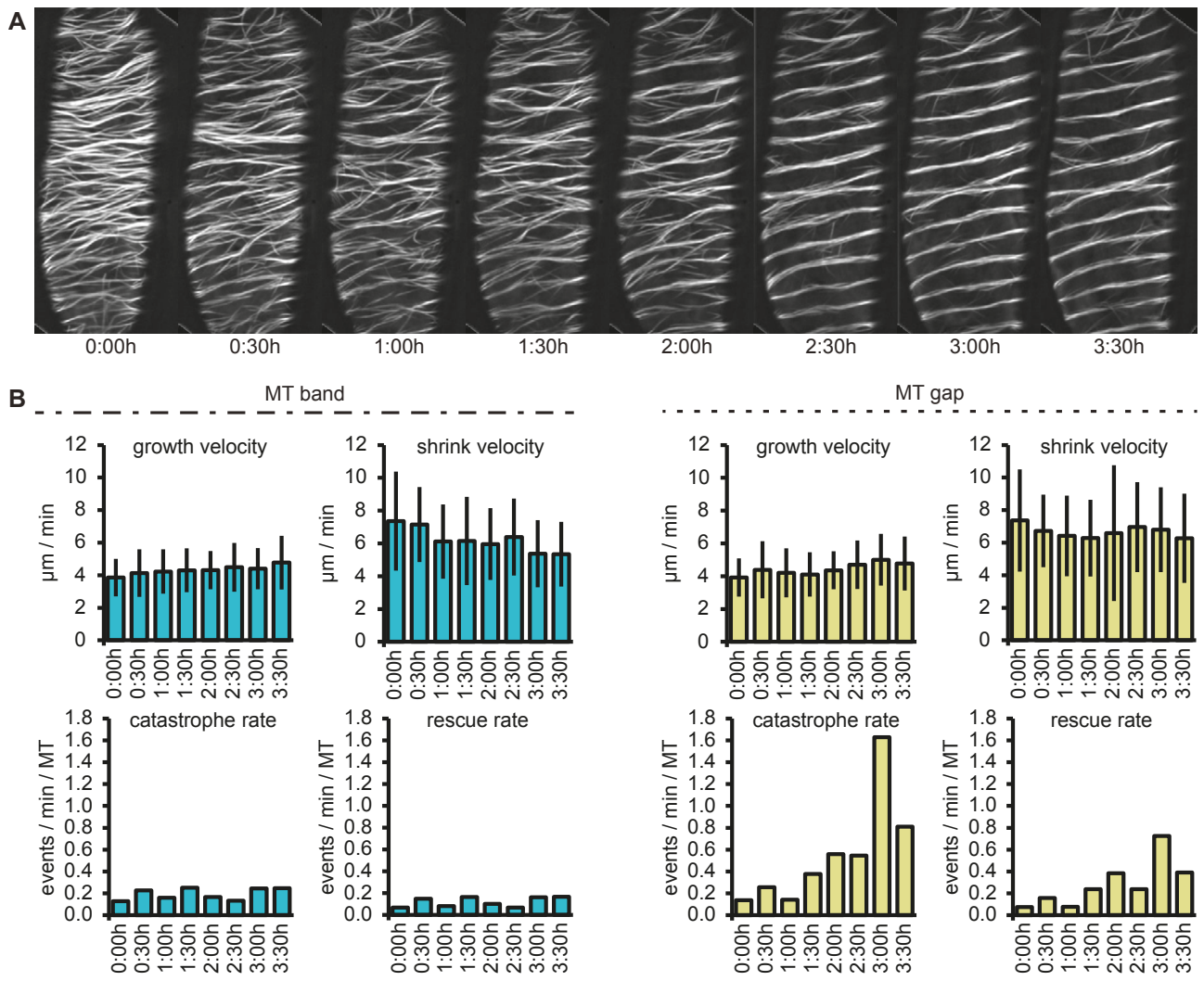

Supplementary Figure S3.2 | A second dataset for microtubule dynamics during microtubule rearrangements in the VND7-driven secondary wall program. (A) Average images of a threeand-a-half-hour time-lapse of YFP-labelled microtubules in an induced hypocotyl cell. Scale bar $=5 \mu \mathrm{m}$. (B) Analysis of microtubule (MT) dynamics parameters split into microtubule band (cyan) and microtubule gap (yellow) areas.

\section{Acknowledgement}

Kris van 't Klooster was supported by the SRON space research program with project number GO-MG/15, which is financed by the Netherlands Organization for Scientific Research (NWO). We would like to thank the Wageningen Light Microscopy Centre (WU) and the Biological Optical Microscopy Platform (BOMP, UoM) for the use of their facilities and Bela Mulder for helpful discussions. Staffan Persson was supported by R@MAP Professor funds at UoM and by an ARC DP grant (DP150103495). 


\section{Chapter}

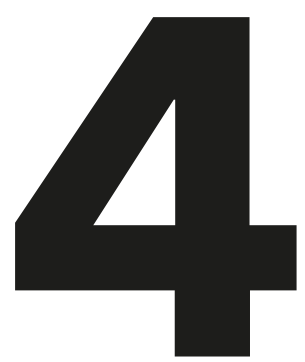

Spatial manipulation of microtubule catastrophe rate causes pattern formation of stochastically simulated microtubules

Kris van 't Klooster, Jasper van der Gucht, Bela Mulder 


\section{Introduction}

Plant development requires control of spatial and temporal processes at the cell level to facilitate differentiation at the tissue level. The cortical microtubule array is important for spatial processes in plant cells ${ }^{83}$. Xylem differentiation is such a plant developmental process which requires spatial patterning of the cortical microtubule array (chapter 1 \& 2). To understand xylem differentiation, it is important to understand patterning of the cortical microtubule array at the cell level. Here we approach pattern formation of the cortical microtubule array during xylem differentiation via computer simulations.

Microtubules in the cortical array consist of tubulin dimer proteins which polymerise or depolymerise, resulting in growth and shrinkage ${ }^{84}$. The switch from a growing to a shrinking state is defined as a catastrophe and the switch from shrinking to growing as a rescue ${ }^{27}$. The stochastic switching between growing and shrinking states of microtubules is defined as dynamic instability. Together with the possibility to nucleate new microtubules, the ingredients are present for a dynamic microtubule array which is able to self-organize ${ }^{35}$. Microtubule associated proteins (MAPs) can alter for example the growth speed, shrink speed, catastrophe rate, rescue rate and nucleation rate 85,86 . Therefore, MAPs can alter dynamic instability properties and direct the microtubule array into different configurations. Understanding microtubule dynamics is important to understand how the microtubule array changes. This can guide the search for cell mechanisms which control microtubule array changes and thereby improve the understanding of spatial processes in plant development.

There is order in microtubule arrays at different levels. Ascending in scale we encounter order with atoms forming molecules, molecules forming amino acids, amino acids forming proteins and proteins forming polymers. A polymer of tubulin dimers forms a single microtubule. Multiple microtubules together make up the cortical microtubule array of a plant cell. Microtubules in the array can be dispersed randomly at the plasma membrane or constitute some order due to their orientation relative to surrounding microtubules and to the shape of the cell. Low order is present in an array with many microtubule orientation angles and high order when few microtubule orientation angles are present. This description of order can be captured quantitatively in an order parameter ${ }^{58}$. Another type of order in microtubule arrays is the optical density, as observed via microscopy. The surface spread of the microtubule array can be even, or highly localized to certain areas of the cell surface. In the latter case, patterns can emerge if there is some degree of periodicity in the optical density. Investigating pattern formation of the microtubule array is of cardinal importance to understand plant developmental processes that are occurring in the chaotic environment of a biological cell. 
To investigate pattern formation of the microtubule array we use the developmental pathway of xylem formation in plants. Highly specialized xylem vessel cells are responsible for the water transport from root to shoot. During cell differentiation of these vessels, the microtubule array is reorganized and a patterned microtubule array with transverse microtubule bands is observed experimentally (Fig. 4.2B; chapter 2 \& 3). The banded microtubule array underlies periodic cell wall thickenings ${ }^{78}$ and when the microtubule dynamics are changed by drugs during xylem differentiation, the patterned deposition of these cell wall thickenings is affected (chapter $2 \& 3$ ). Therefore, the microtubule array is considered to act as a template for patterned deposition of cell wall thickenings. Time series of microtubule dynamics during xylem differentiation show that the microtubule array reorganizes into a pattern of multiple transverse microtubule bands, intermitted by microtubule gap zones (Fig. 4.2B; chapter $2 \& 3$ ). The microtubule density in the microtubule band is high whereas it is low in the microtubule gap zone. In this chapter we investigated microtubule pattern formation from a theoretic perspective using stochastic computer simulations of microtubules. We show that changing the catastrophe rate in a spatially non-uniform manner is sufficient to recreate the experimentally observed bands and gaps of microtubules in plants cells of differentiating xylem vessels.

\section{Materials and Methods}

\section{Microtubule simulation algorithm}

We performed stochastic simulations of dynamic cortical microtubules represented as line-like objects, as microtubules have a long persistence length and can therefore be considered as rigid structures ${ }^{87}$. The simulation space consists of a cylindrical geometry with length $80 \mu \mathrm{m}$ and circumference $40 \mu \mathrm{m}$. Cortical microtubules in cells can be thought of as existing in a pseudo-2D space, as they are connected to the plasma membrane and the volume between the vacuole and the plasma membrane is small ${ }^{31}$. Therefore we simulated the cylinder as a $2 \mathrm{D}$ surface with periodic boundaries conditions. There is a fixed number of microtubules per simulation, all starting out in a zero length dormant state from which they are nucleated into a growing state. At a nucleation event the location and orientation of the new microtubule are selected randomly. The parameters of microtubule (MT) dynamics are based on previous simulation work ${ }^{58,88}$ with growth speed $\mathrm{V}^{+}=4.7 \mu \mathrm{m} \mathrm{min}^{-1}$, shrinkage speed $\mathrm{V}^{-}=9.8 \mu \mathrm{m} \mathrm{min}^{-1}$, catastrophe rate $R_{c}$ $=0.24 \mathrm{~min}^{-1}$, rescue rate $R_{r}=0.4 \mathrm{~min}^{-1}$ and nucleation rate $R_{n}=0.12 \mathrm{MT}^{-1} \mathrm{~min}^{-1}$. These simulations were constructed bottom-up with the focus on investigating the 
minimal requirements for microtubule patterning relating to protoxylem formation, so that minus-end treadmilling and microtubule- microtubule interactions are not implemented at this level. Treadmilling is the phenomenon in which a microtubule grows at the plus end and shrinks at the minus end, resulting in the movement of the microtubule away from the nucleation location.

\section{Spatially non-uniform catastrophe rate based on a sinusoid function.}

To mimic the experimentally observed microtubule pattern during protoxylem formation (Fig. 4.2), we apply a spatially non-uniform enhancement of catastrophes. microtubule tips encounter a spatially non-uniform catastrophe rate based on a sinusoidal function

$$
R_{c}(z)=\frac{1}{2}(\rho+1) R_{c_{-} \min }+\frac{1}{2}(\rho-1) R_{c_{-} \min } \sin \left(2 \pi \frac{\mathrm{z}}{\lambda}\right)
$$

The catastrophe rate of a microtubule tip at $R_{c}(z)$ depends on the simulated cylinder height $(Z)$, the wavelength $(\lambda)$, the minimum catastrophe rate $\left(R_{c_{\text {mmin }}}\right)$ and a catastrophe rate amplifier $(\rho)$. The maximum catastrophe rate $\left(R_{c_{-} \text {max }}\right)$ is defined as follows

$$
R_{c_{-} \max }=\rho R_{c_{-} \min } \quad(\rho \geq 1)
$$

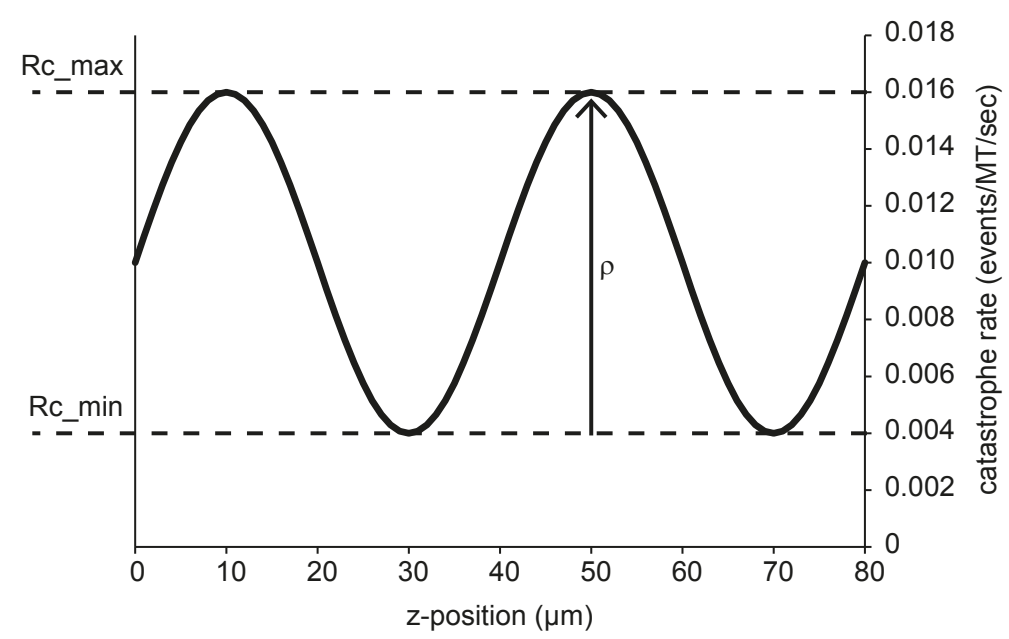

Figure 4.1 | Sinusoidal change of catastrophe rate. The catastrophe rate is amplified according to equation 1 with a catastrophe rate amplifier $(\rho)$. This example is with $R_{c_{-} \min } 0.004$ (events/MT/ $\mathrm{sec}$ ) and a $\rho$ of 4 , resulting in a $R_{c_{\text {max }}}$ of 0.016 (events/MT/sec). The wavelength $\lambda$ is set to $40 \mu \mathrm{m}$, which is $1 / 2$ the height of the simulated cylinder, in order to create 2 peaks of $R_{c_{\text {max }}}$. 
The orientation of the sinusoidal function (equation 1 ) is parallel to the long axis of the simulated cylinder ( $Z$ ) in order to create transverse bands with low and high catastrophe rates perpendicular to the long axis of the simulated cylinder (Fig. $4.2 \mathrm{C}$ ). To reduce the processing time required for the non-uniform catastrophe rate, the sinusoidal function (equation 1 ) was sampled with 80 bins (B), giving a bin size of $1 \mu \mathrm{m}(\delta)$. The wavelength $(\lambda)$ is set to $40 \mu \mathrm{m}$, which is $1 / 2$ the simulated cylinder height, in order to create 2 peaks where $R(\mathrm{z})$ reaches $R_{c \_ \text {max }}$ (Fig. 4.1). At these $R_{c \text { max }}$ peaks, regions appear with a low microtubule density, representing experimentally observed microtubule gaps in between microtubule bands (Fig. 4.2). The default values for the stochastic simulations in this chapter can be found in Table 4.1.

Table 4.1 | Simulation parameters of microtubules (MT) with default values.

\begin{tabular}{lll}
\hline Parameter & Description & Value \\
\hline $\mathrm{V}^{+}$ & Growth speed & $4.7 \mu \mathrm{min}^{-1}$ \\
$\mathrm{~V}^{-}$ & Shrink speed & $9.8 \mu \mathrm{min}^{-1}$ \\
$R_{r}$ & Rescue rate & $0.4 \mathrm{~min}^{-1}$ \\
$R_{c}$ & Catastrophe rate & $0.24 \mathrm{~min}^{-1}$ \\
$R_{c_{\text {min }}}$ & Minimum catastrophe rate & $0.24 \mathrm{~min}^{-1}$ \\
$R_{c_{-} \max }$ & Maximum catastrophe rate & $0.24-1.92 \mathrm{~min}^{-1}$ \\
$\rho$ & Catastrophe rate amplifier & $1-8$ \\
$R_{n}$ & Nucleation rate & $0.12 \mathrm{MT}^{-1} \mathrm{~min}^{-1}$ \\
$\mathrm{Z}$ & Simulation cylinder height & $80 \mu \mathrm{m}$ \\
$\lambda$ & Wavelength & $40 \mu \mathrm{m}$ \\
$\delta$ & Bin size sinusoidal function & $1 \mu \mathrm{m}$ \\
$\mathrm{B}$ & Number of bins & 80 \\
\hline
\end{tabular}


A
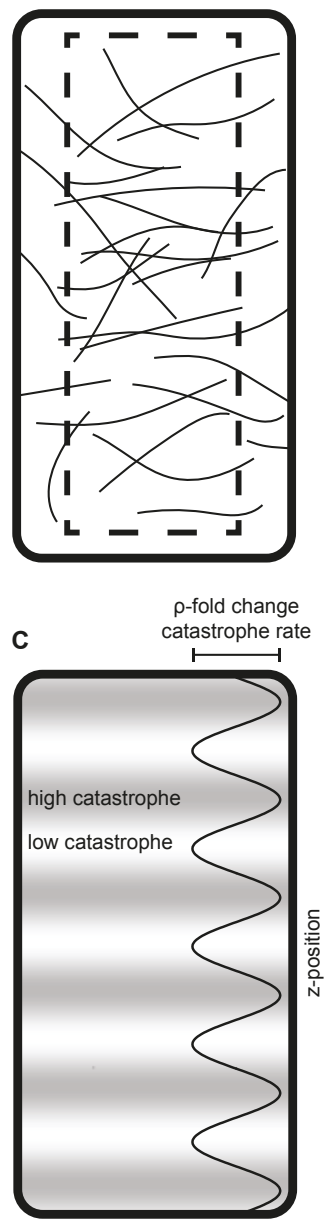

B

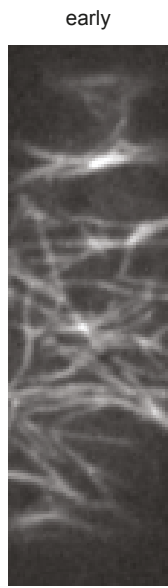

D

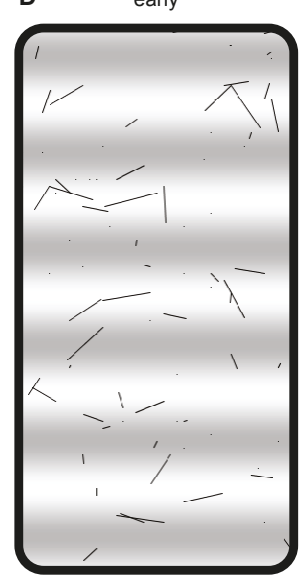

mid

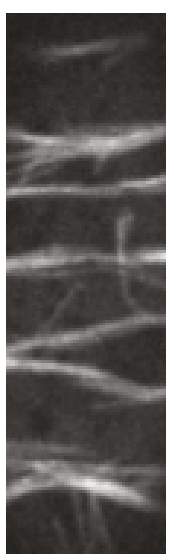

mid

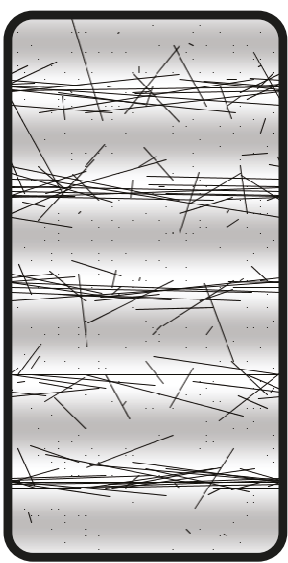

late

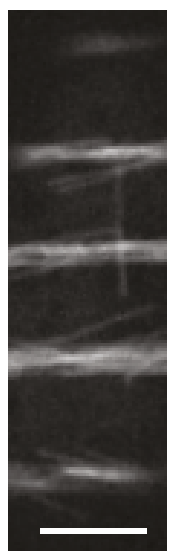

late

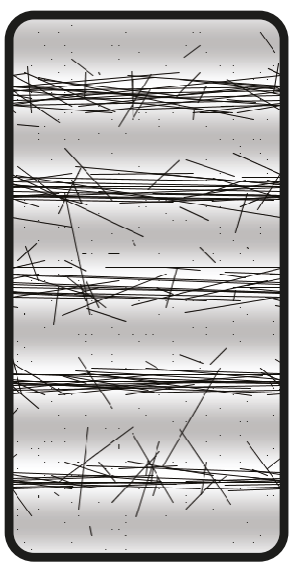

Figure 4.2 | Experimentally observed microtubule band formation in xylogenesis of plants is recreated in silico by changing the catastrophe rate dependent on the z-position of the growing tip of microtubules. (A) Model of one Arabidopsis hypocotyl cell with cortical microtubules and a field of view resulting from spinning disk microscopy (dashed box). (B) Induced xylogenesis results in a major reorganization of the microtubule array in hypocotyl cells of Arabidopsis. The cortical microtubule array reorganizes from an interphase array (early) to an array with microtubule bands intermitted by regions with a reduced density of microtubules (late). Early, mid and late images are single focal planes. Scale bar $=5 \mu \mathrm{m}$. (C) Model of changed catastrophe rate dependent on the z-position of the growing tip of microtubules. The sinusoidal curve changes the catastrophe rate depending the $\rho$-fold change of the base catastrophe rate. In the white areas the $R_{c \text { min }}$ (low) is in effect, in the grey areas the $R_{c \text { min }}$ is amplified $\rho$-fold resulting in an increased catastrophe rate up to $R_{c_{\text {max }}}$ (high). The transition from low to high catastrophe rate is implemented via equation 1 to generate a smooth transition. (D) Time snapshots of stochastic simulation of dynamic microtubules in silico with the sinusoidal catastrophe rate in effect with $\rho$ $=4$ and total simulation time $2 \mathrm{~h} 46 \mathrm{~m}$. 


\section{Results}

\section{A spatial alternation of high and low catastrophe rates results in microtubule bands}

Reorganization of the microtubule array occurs in the process of protoxylem formation (chapter $2 \& 3$ ). The microtubule array reorganizes from an interphase microtubule array into a highly patterned array with transverse microtubule bands. The cortical microtubules are dispersed over the plasma membrane (Fig. 4.2B; 'early'), followed by a transition with the first microtubule bands and gaps appearing (Fig. 4.2B; 'mid'), resulting in a high density of microtubules in bands perpendicular to the long cell axis with regions in between with a reduced density (Fig. 4.2B; 'late'). In order to understand the transition of the microtubule array during protoxylem formation we used stochastic simulations to recreate the experimentally observed microtubule pattern. We tested if spatial variation of one parameter is sufficient to generate a banded microtubule pattern. To mimic the observed banded pattern in experiments (chapters $2 \& 3$ ), we applied a nonuniform enhanced catastrophe rate based on a sinusoidal function (equation 1). Growing microtubule-tips encounter a catastrophe rate dependent on the z-position of the simulated cylinder. The catastrophe rate alternates between $R_{c \_ \text {min }}$ and $R_{c \text { max }}$ according to the sinusoidal function (Fig. 4.1). With a 4-fold increase of $R_{c \text { min }}$ the stochastic simulation of microtubules results in a banded pattern with gap regions in between with a decreased microtubule density (Fig. 4.2D) that mimics the experimental observation (Fig. 4.2B).

\section{The mean microtubule length is non-uniformly affected by the sinusoidal modified catastrophe rate}

We analyse the mean length $(\bar{l})$ of microtubules as described by Mulder $(2012)^{80}$ to understand the effect of the sinusoidal changed $R_{c}$. The mean length is dependent on the inverse of the difference between the ratios $R_{c} / \mathrm{V}^{+}$and $\mathrm{R}_{\mathrm{r}} / \mathrm{V}^{-}$

$$
\bar{l}=\frac{1}{\frac{R_{c}}{\mathrm{~V}^{+}}-\frac{R_{r}}{\mathrm{~V}^{-}}}
$$

The according to equation 3 is reached given sufficient time and as microtubule dynamics parameters remain constant. In our stochastic simulation these parameters are not constant and therefore the microtubule lengths is affected by the history of growth. A microtubule of a certain length encountered different $R_{c}$ rates depending on the starting position and orientation. The calculated $\bar{l}$ 
presented is therefore an indication to assess if the local microtubule dynamics parameters promote long microtubules or not (Fig. 4.3 \& S4.1). All parameters are set constant except for $\rho$ which consequently affects the mean length $(\bar{l})$. The calculated $\bar{l}$ indicates if the parameter set is driving towards long microtubules (high $\bar{l}$ ) or towards short microtubules (low $\bar{l}$ ). The magnitude of the effect on $\bar{l}$ depends on the $\rho$-fold change of $R_{c \text { min }}{ }^{\prime}$ which depends on the z-position of the simulated cylinder, the ratio between $R_{c_{-m i n}}$ and the other parameters $\mathrm{V}^{+}, \mathrm{V}^{-}$and $R_{r}$. If $\rho=1$, there is no change in $R_{c}$ (Fig. 4.3A), resulting in a uniform $\bar{l}$ over the simulated cylinder. If $\rho>1, \bar{l}$ is non-uniformly affected according to equation 1 , resulting in a $\bar{l}$ for each $z$-position on the simulated cylinder which depends on according to equation 3. Thus, a microtubule will encounter different $R_{c}$ rates dependent on the z-position of the growing microtubule tip (Fig. 4.2C). A 2-fold increase of $R_{c}$ results in a reduction of the minimum $\bar{l}$ from $101.85 \mu \mathrm{m}$ (Fig 4.3A, $\rho$ $=1$ ) to $16.36 \mu \mathrm{m}$ (Fig. 4.3B; $\rho=2$ ). The minimum $\bar{l}$ with $\rho=4$ is even lower, $6.11 \mu \mathrm{m}$ (Fig. 4.3C). Thus, a 2-fold and 4-fold increase of $R_{c}$ result in a 6.2-fold and 16.7-fold reduction of $\bar{l}$, respectively according to equation 3 . The sinusoidal modification of $R_{c}$ results in a high $\bar{l}$ around the region where $R_{c}(\mathrm{z})=R_{c \text { min }}$ and a low $\bar{l}$ where $R_{c}(\mathrm{z})=R_{c \text { max }}$ (Fig. 4.3). The spatial distribution of $\bar{l}$ is non-uniformly affected by the sinusoidal change in $R_{c^{\prime}}$ with in a steep maximum $\bar{l}$ around z-positions 30 and 70 $\mu \mathrm{m}$ (Fig. 4.3). We quantified the $\bar{l}$ in stochastic simulations in the range $\rho=1-8$ (Fig. 4.4), confirming the predicted maximum $\bar{l}$ around z-positions 30 and $70 \mu \mathrm{m}$ (Fig. 4.3). Over time the microtubule density will be high around these maxima with a wide region in between with a low microtubule density.

\section{A 2-fold sinusoidal increase in catastrophe rate is sufficient to create microtubule gap regions with a low density between microtubule bands with a high density}

The mean length is spatially affected by the sinusoidal change of $R_{c}$. We quantified the effect on the microtubule length density in stochastic simulations in the range of $\rho=1-8$ (Fig. 4.5A). A 2-fold increase of $R_{c_{-} \text {min }}$ resulted in a microtubule pattern with alternating regions of high and low microtubule densities (Fig. 4.5A; $\rho=2$ ), with a minimum observed microtubule length density of $11.21 \mu \mathrm{m} \mathrm{bin}{ }^{-1} \pm 1.63$ (Fig. 4.5B). A 4-fold increase of $R_{c_{-} \text {min }}$ resulted in a microtubule gaps in which the microtubule length densities approaches $0 \mu \mathrm{m} \mathrm{bin}^{-1}($ Fig. 4.5A; $\rho=4)$. These results show that spatially changing the $R_{c}$ according to equation 1 is a suitable method to recreate the experimentally observed microtubule patterns in plant cells. This is also reflected in the reduced minimum observed microtubule density at $\rho=3$ with $2.12 \mu \mathrm{mbin}^{-1} \pm 1.63$ and at $\rho=4$ with $0.32 \mu \mathrm{m} \mathrm{bin}^{-1} \pm 0.51$ (Fig. 4.5B). 

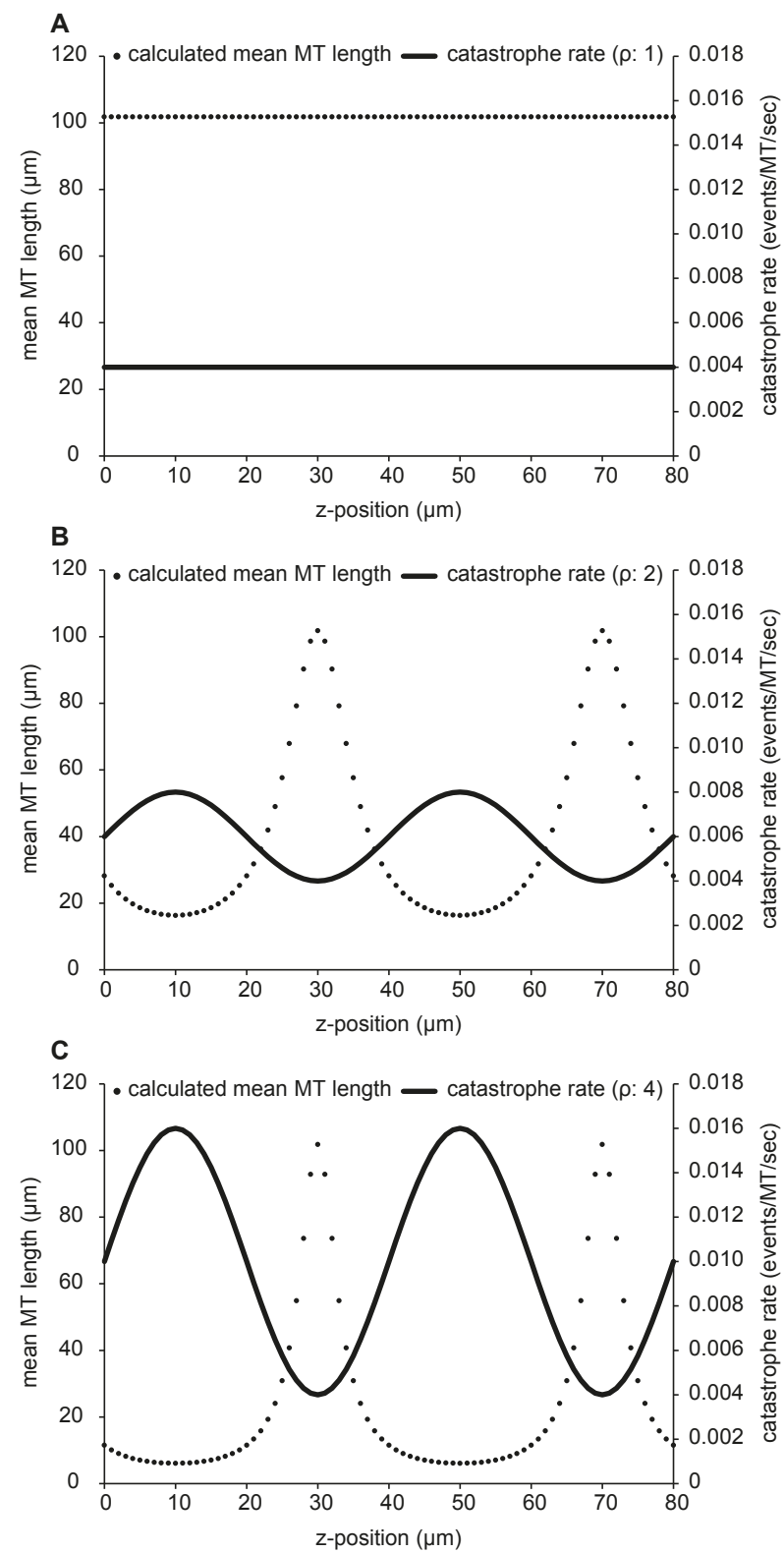

Figure 4.3 | The mean microtubule length is non-uniformly affected by the sinusoidal catastrophe rate change. (A) The mean microtubule (MT) length in a system can be calculated based on the catastrophe rate, rescue rate, growth speed and shrink speed. In (A) the $\rho$-fold change is 1 , indicating a constant catastrophe rate (line) resulting in a uniform mean microtubule length (dots). (B) With $\rho: 2$ the catastrophe rate alternates between 0.004 and 0.008 (events/MT/ $\mathrm{sec}$ ) according to a sinusoidal function with 2 wavelengths. The mean length is decreased nonuniformly dependent on the z-position. (C) With $\rho: 4$ the catastrophe rate alternates between 0.004 and 0.016 (events/MT/sec). As in (B) the mean length is non-uniformly affected with a narrow peak of an increased calculated mean microtubule length at z-position $30 \& 70 \mu \mathrm{m}$, whereas at the other z-positions the mean microtubule length is decreased. 
A
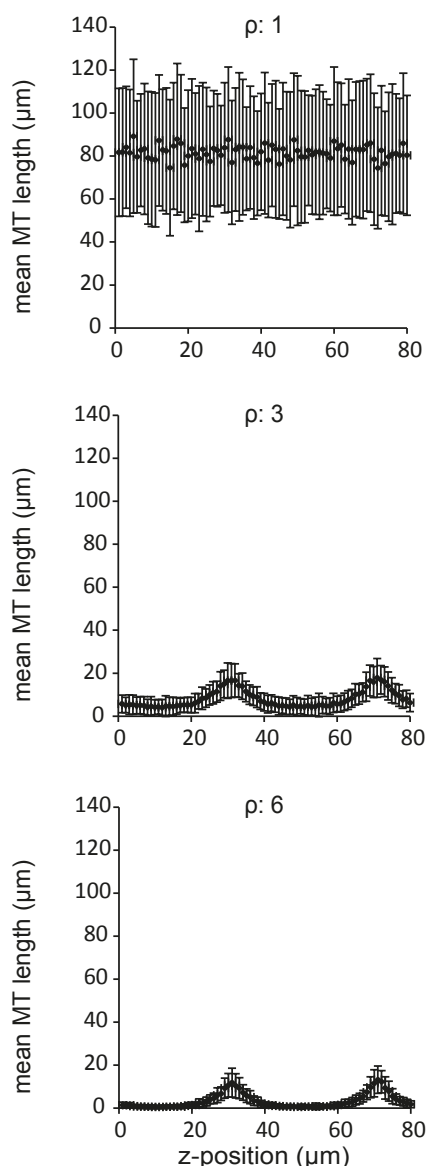

B
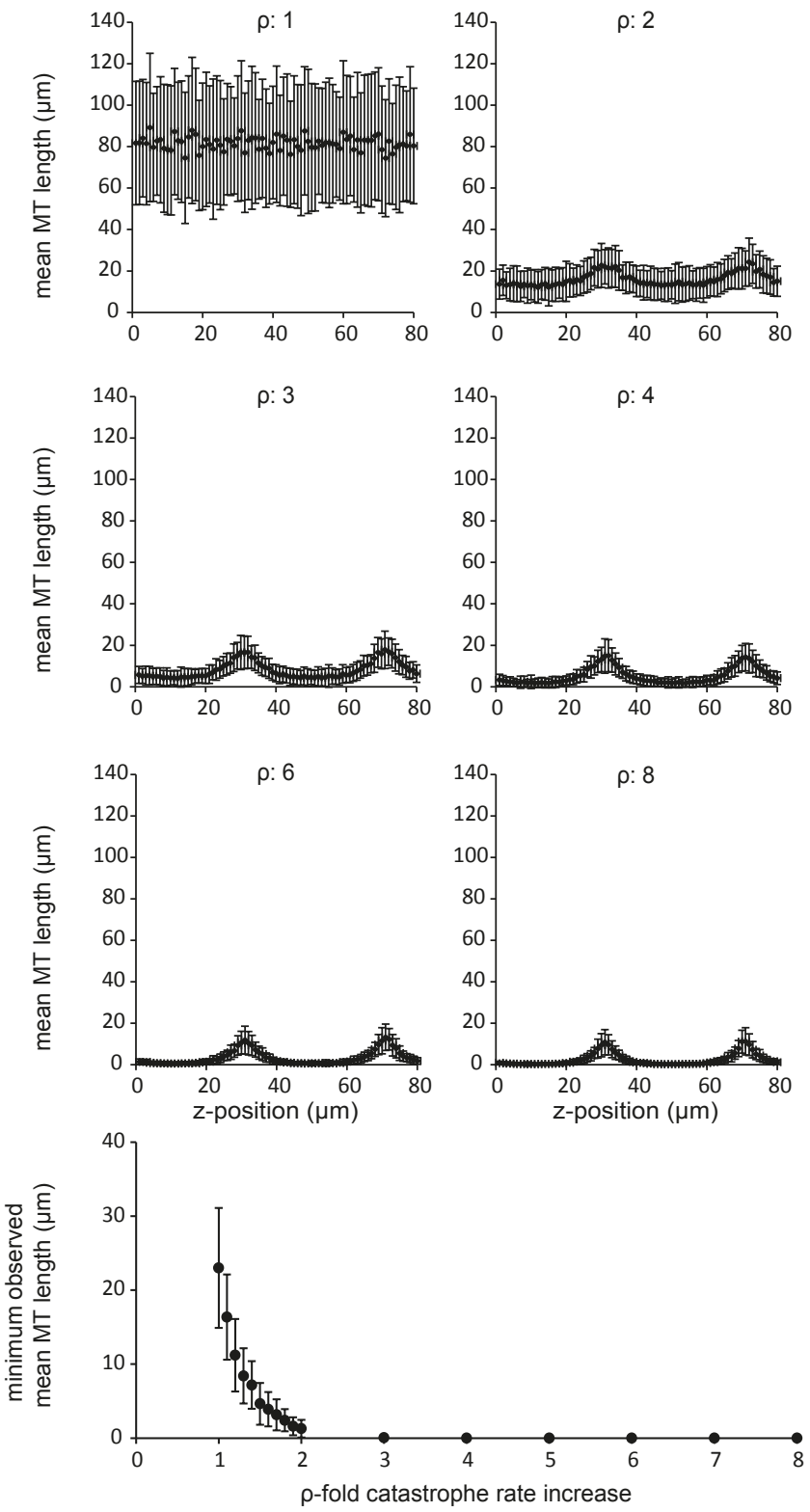

р: 4
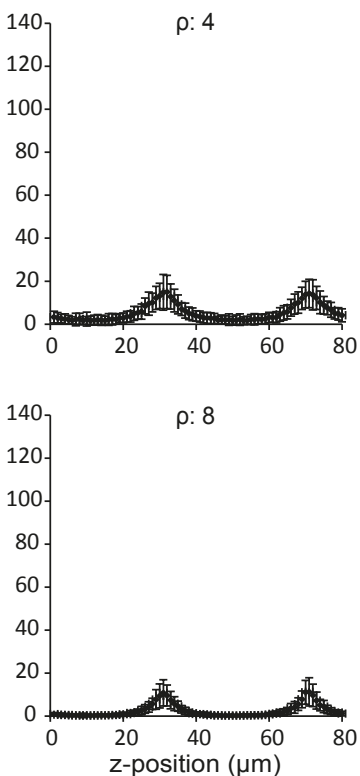

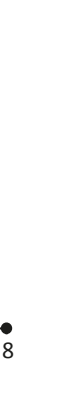

Figure 4.4 | The sinusoidal increased catastrophe rate reduces the mean microtubule length non-uniformly over space. (A) The effect of the $\rho$-fold sinusoidal increase of the catastrophe rate on the mean microtubule (MT) length is plotted for the range $\rho: 1-8$. At $\rho: 4$ the regions in between the peaks approaches $0 \mu \mathrm{m} /$ bin mean MT length, indicating that microtubules in these regions are very short. (B) The minimum observed mean MT length is plotted for the range $\rho: 1-8$. As already observed in (A), at $\rho: 4$ the minimum mean MT length approaches $0 \mu \mathrm{m}$. The strong decrease between $\rho: 1-2$ indicate that a small increase in catastrophe rate has a strong effect on the mean MT length. $N=1000$ simulation runs. Error bars indicate standard deviations. Base catastrophe rate is 0.004 (events/MT/sec). Data is collected after $24 \mathrm{~h}$ of simulated time. 
A
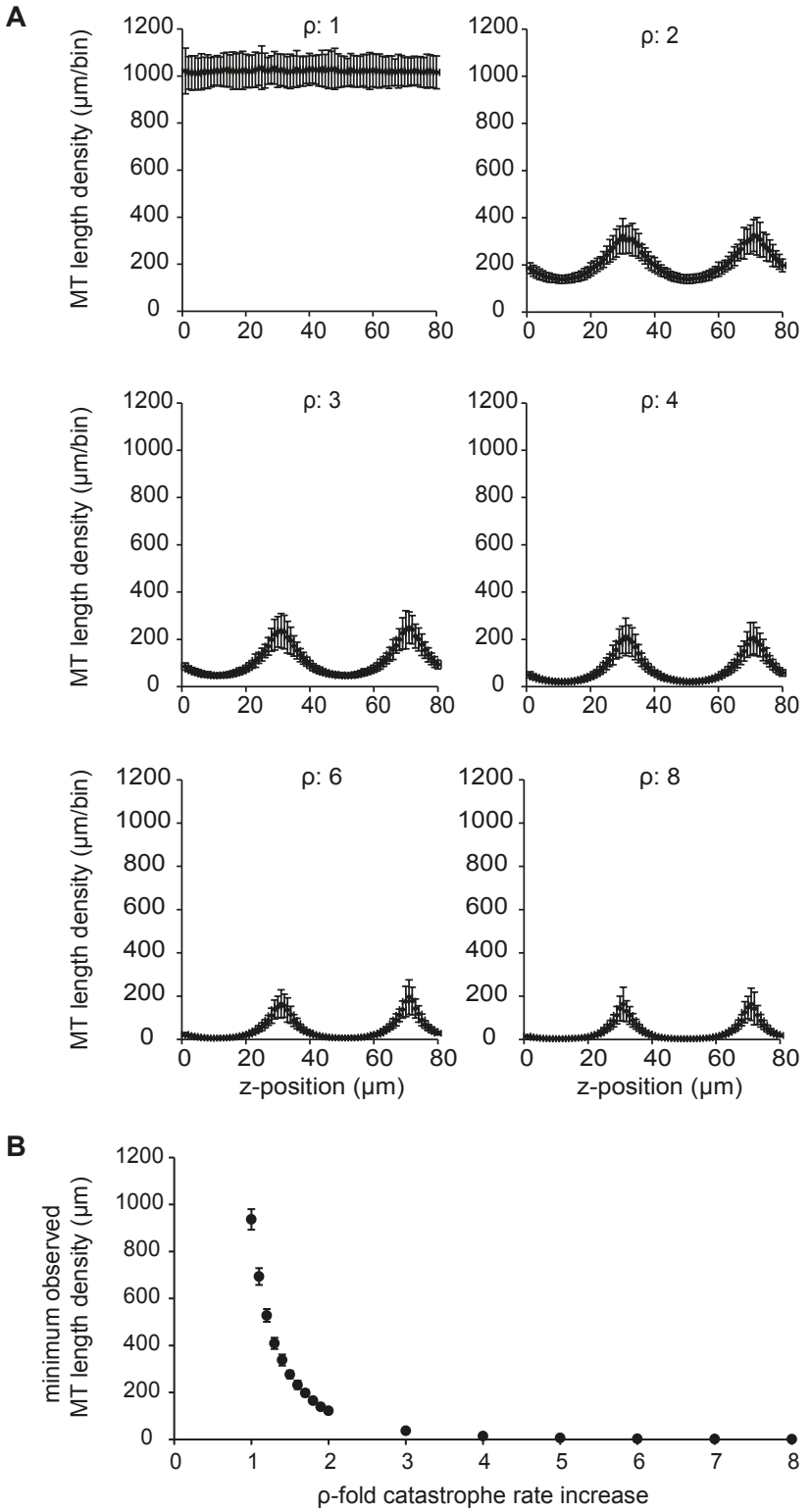

Figure 4.5 | A 2-fold increase of the catastrophe rate is sufficient to create low microtubule length density regions between microtubule bands in stochastic microtubule simulations. (A) The $\rho$-fold sinusoidal increase of the catastrophe rate is implemented in a stochastic microtubule simulation to analyse the effect on microtubule (MT) length density in the range $\rho: 1-8$. At $\rho: 2$ the microtubule (MT) length density shows clearly two peaks at $30 \& 70 \mu \mathrm{m}$ which are separated by regions with a decreased MT length density. At $\rho: 4$ the regions in between the peak approaches $0 \mu \mathrm{m} / \mathrm{bin}$ MT length density, indicating that these regions are devoid of microtubules. (B) The minimum observed MT length density is plotted for the range $\rho: 1-8$. As already observed in (A), at $\rho: 4$ the minimum MT length density approaches $0 \mu \mathrm{m}$. The strong decrease between $\rho: 1-2$ indicates that a small increase in catastrophe rate has a strong effect on the MT length density. $\mathrm{N}=1000$ simulation runs. Error bars indicate standard deviations. Base catastrophe rate is 0.004 (events/MT/sec). Data is collected after $24 \mathrm{~h}$ of simulated time. 


\section{Discussion}

A simple model can aid in the understanding of a complex biological process. Therefore we investigated pattern formation of the microtubule array during protoxylem formation using stochastic computer simulations. We show that a spatial modification of a microtubule parameter, the catastrophe rate $\left(R_{c}\right)$, is sufficient to drive pattern formation resembling the transverse banding of microtubules observed experimentally (chapter $1 \& 2$ ). The sinusoidal changes in mean length $(\bar{l})$ drive the formation of periodic microtubule bands and microtubule gaps. A mechanism based on a locally adapted, as we have employed here, does not necessarily need to function via the $R_{c^{\prime}}$ but could equally function via $R_{r^{\prime}} \mathrm{V}^{+}$or $\mathrm{V}^{\text {; }}$, or a combination of the parameters in equation 3 , as long as the effect on $\bar{l}$ is similar. We calculate here that a 2 -fold increase in $R_{c_{-\min }}$ results in 6.2-fold decrease in calculated $\bar{l}$ according to equation 3, which is sufficient to generate microtubule gaps with a low microtubule density between microtubule bands with a high microtubule density. The experimental data from chapter 3 shows a 2-3-fold increase in $R_{c}$ over time in microtubule gaps (Fig. 3.3D), indicating that a 2 -fold change is feasible during protoxylem formation. The mean length distribution in a microtubule array is difficult to assess experimentally, as the minus and plus ends of microtubules are difficult to find with multiple microtubules in bundles and microtubules spanning the complete field of view in microscope images. By using the experimentally determined microtubule dynamicity parameters as input for equation 3 , the change in $\bar{l}$ is calculated in microtubule gaps over time, and shows a 8.1 -fold decrease between the initial $\bar{l}$ of $38.1 \mu \mathrm{m}$ to the final $4.7 \mu \mathrm{m}$ when the banded microtubule pattern is established (chapter 3; Fig. 3.3E). The changes in $R_{c}$ and the calculated $\bar{l}$ are in the same order of magnitude when compared to the experimental work (chapter 3 ).

Reality is more complex than our simulations. In chapter 3 we showed that besides a modified $R_{c_{1}}$ also $R_{r}$ is changed during protoxylem formation (Fig. 3.3). Furthermore, there is a temporal discrepancy between pattern formation in the experiments and the simulations. We experimentally observed a relative quick pattern establishment period of about 1 hour (chapter 3; Fig. 3.2D), compared to the multiple day process of protoxylem formation. The stochastic simulations span a period of 24 hours of simulated time. To address the differences between our simulations and reality, the discrepancies should be investigated. A next step could be to include the assessed experimental microtubule dynamicity rates as temporal changes in the simulations. A different direction could be to include different nucleation modes, which can drastically alter the speed of microtubule array self-organization ${ }^{88}$. Another promising direction is to investigate the effect 
of the initial order of the microtubule array before the onset of the patterning mechanism, both experimentally and via simulations. The stochastic simulations in this chapter are initially free of microtubules and require time to be populated with microtubules. The sinusoidal change in $R_{c}$ is constantly present, but only when the microtubule density is high the sinusoidal differential $R_{c}$ is able to pattern the microtubule array. In the future, the patterning mechanism could be activated after a certain microtubule density is reached, and then the effect of an initial microtubule array orientation on patterning speeds can be assessed. Furthermore, it is interesting to establish the pattern and then remove the sinusoidal effect to assess how stable to pattern is.

In our simulations we assume that a spatial pattern is present, which provides a template for the locally changed microtubule dynamics. How this pattern is established remains an important question to be answered in order to understand microtubule array reorganization during protoxylem formation. In metaxylem, consisting of transport vessels which differentiate in non-elongating plant tissues after growth, pattern establishment is thought to be dependent on a reactiondiffusion based mechanism ${ }^{53}$. A reaction-diffusion mechanism is a multiplecomponent system in which components react with each other coupled by different diffusion speeds, resulting in periodic patterns of local activation and deactivation of one of the components in the system ${ }^{19,20}$. In metaxylem patterning ROPGEF4 and ROPGAP3 act as activator and inhibitor, respectively, on the GTPase ROP11, creating active ROP11 plasma membrane domains ${ }^{53}$. Active ROP11 recruits the microtubule depolymerising MAPs MIDD1 and Kinesin13A, rendering the plasma membrane domain free of cortical microtubules ${ }^{54,55}$. During metaxylem differentiation no secondary cell wall material is deposited at these microtubule free regions resulting in the typical pitted pattern of metaxylem vessel elements ${ }^{53}$. The pitted pattern is not the only pattern present in xylem, therefore we try to understand how differentiating protoxylem vessel elements form rings and spirals of secondary cell wall thickenings to improve our understanding of pattern formation in xylem formation.

Our results show that stochastically simulated growing and shrinking microtubules with a spatial differential $R_{c}$ pattern recreates the experimentally observed periodic pattern of microtubule bands and gaps. The required change in $R_{c}$ to form a pattern is in a similar order of magnitude as experimentally observed. This theoretical approach using stochastic computer simulations can function as a starting point for further investigations of pattern formation of the microtubule array during xylem formation, both experimentally and theoretically. 


\section{Supplementary Figure}
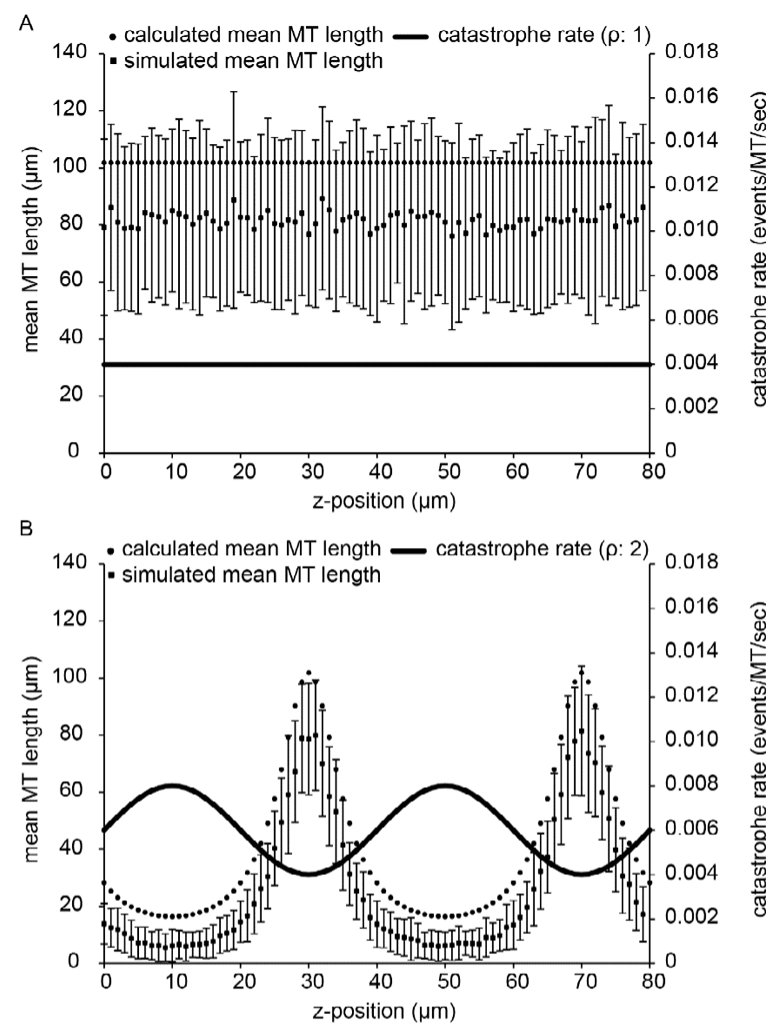

C

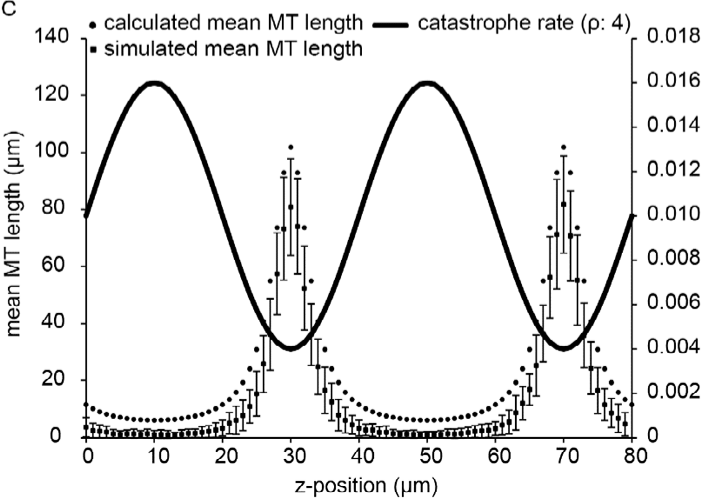

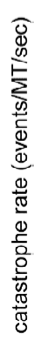

Supplementary Figure S4.1 | Validation of the simulated mean microtubule length and the sinusoidal catastrophe rate change. The squares indicate the simulated mean microtubule (MT) length $(n=100)$. The angle of microtubules in this simulation is fixed to 0 degrees, indicating a perfect transverse alignment perpendicular to the sinusoid. This allows the microtubules to reach the theoretic mean $\mathrm{MT}$ length based on the catastrophe rate at that particular z-position. Microtubules growing in random orientations encounter a changing catastrophe rate depending on the z-position of the growing tip, this will reduce the theoretic mean length of microtubules. Error bars indicate standard deviations. Data is collected after $24 \mathrm{~h}$ of simulated time. 


\section{Acknowledgements}

I would like to thank Eva Deinum for helpful discussions. 



\section{Chapter}

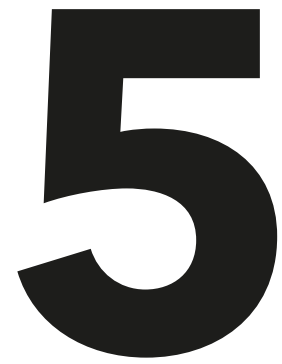

\section{Patterned secondary cell wall deposition during protoxylem formation proceeds normally under simulated altered gravity conditions}

Kris van 't Klooster, Jack van Loon, Tijs Ketelaar 


\section{Introduction}

Plants have a sessile life strategy that allowed plants to evolve intriguing adaptations to survive. The plant cell wall that surrounds cells to provide support and rigidity is such an adaptation. A bone skeleton as we know it in animals is absent in plants, and therefore plant cell walls are required to cope with the environmental factor gravity. The direction of gravity is constant and the plant's response to this directional cue is termed gravitropism ${ }^{89}$. Likewise, the force of gravity is constant and the combination of plant traits to cope with the force of gravity is known as gravity resistance ${ }^{89}$.

Water and nutrient transport from the root to shoot of plants is counteracted by gravity and higher plants thus need to cope with this issue. To counteract gravity during water transport, higher plants evolved xylem tissue, a continuous tubular transport system from root to shoot that consists of connected vessel elements, cells that undergo a differentiation program that involves the deposition of cell wall reinforcements, perforation of interconnecting walls and finally programmed cell death ${ }^{6,66}$. The cell wall reinforcements are characterized by intricate patterned secondary cell wall thickenings which are required to resist forces present during evaporation driven transport ${ }^{6}$. The plant cell wall consists of a matrix of polysaccharides, with crystalline cellulose microfibrils as the load-bearing component ${ }^{2}$. Like in primary (expanding) cell walls, cortical microtubules recruit cellulose synthase complexes to the plasma membrane $39,40,52$ that synthesize the cellulose microfibrils which are incorporated in the existing cell wall matrix ${ }^{42}$. Cortical microtubule arrays in developing xylem cells resemble the secondary cell wall patterns ${ }^{52,78}$. Therefore, the cortical microtubule array is involved in directing the patterned secondary cell wall deposition during xylem formation (chapter 2). During protoxylem formation the cortical microtubule array reorganizes into an array with microtubule band and gap regions (chapter 2 \& 3 ), with respectively a high and a low density of cortical microtubules that underlies the developing secondary cell wall thickenings ${ }^{52}$. The cortical microtubule array has been hypothesized to self-organize by interactions between individual microtubules and the activity of microtubule associated proteins (MAPS) that modulate the microtubule dynamics ${ }^{28,90,91}$. Although the self-organization of cytoskeletal polymers has received much attention in the last ten years ${ }^{18,19,92-94}$, the organizing principles that govern the reorganization of the microtubule array during protoxylem formation remain elusive.

In gravity research in plants, microtubules are considered as an important connection between gravity sensing of plant cells and remodelling of the plant cell wall ${ }^{95-98}$. This concept is supported by the strong link between microtubules and cellulose synthesis ${ }^{38-40}$, and the observation that the cell wall is remodelled in altered gravity conditions $89,99,100$. One could therefore hypothesize that cell wall remodelling in plants in altered gravity conditions is mediated by an altered microtubule array 
organization. Indeed, the microtubule array was found to be affected in Brassica napus protoplasts, with a reduced microtubule density and fewer parallel microtubule arrays in altered gravity conditions ${ }^{64}$. In contrast to the wall-less protoplast cells, tobacco BY-2 suspension cell cultures with a cell wall, do not show aberrations in microtubule organization when subjected to weightlessness ${ }^{101}$. The different outcomes of these experiments suggests that the cell wall could override gravity induced changes in microtubule organization that are observed in protoplasts.

Since the ability of the cortical microtubule array to respond to altered gravity conditions ${ }^{64}$ appears to be overruled by the cell wall under normal conditions ${ }^{101}$, we set out to test if there are other conditions during which gravitational input in microtubule array organization occurs. We selected the non-equilibrium microtubule array reorganization during protoxylem differentiation as a suitable system to investigate the interaction between the microtubule array and the cell wall, because the microtubule array reorganizes into a predictable pattern which is translated into a cell wall readout (chapter 2 ).

Pattern formation in nature has been proposed to function via a reaction-diffusion mechanisms, including at the cell level where the MIN-system determines polarity in bacteria ${ }^{17,102}$. Pattern formation by reaction-diffusion is based on at least two interacting components with a low and a high diffusion coefficient, resulting in a plethora of patterns depending on the boundary conditions ${ }^{20}$. In reaction-diffusion systems, bifurcation points can exist at which the system has two different stable states $^{62}$. At these points, a small external field, such as gravity, could result in a large response by providing a bias to one of these states ${ }^{61}$. This characteristic of bifurcation points can be used in nature to be able to adapt to changing environments. A reaction-diffusion mechanism has been suggested to influence pattern formation of the microtubule array during metaxylem formation ${ }^{53}$. Metaxylem patterning is based on the activator ROPGEF4 and inhibitor ROPGAP3 which determine the location of the activated GTPase ROP11 in the plasma membrane ${ }^{53}$. ROP11 recruits the microtubule depolymerising proteins MIDD1 and Kinesin-13A, resulting in plasma membrane areas free of cortical microtubules ${ }^{53-55}$. Consequently, no secondary cell wall material is deposited at these activated ROP11 domains, creating the characteristic pitted pattern of metaxylem vessel elements ${ }^{53}$. Microtubules seem to be able to form a barrier that restricts diffusion of ROP11-MIDD1 in the plasma membrane and thereby influences the patterning of ROP11-MIDD1 domains ${ }^{53}$. A shared microtubule patterning mechanism based on reaction-diffusion might be present in both protoxylem and metaxylem formation. If such a reaction-diffusion system is near a bifurcation point under normal conditions, an environmental factor could push the system into another state ${ }^{62,103}$. We investigate if gravity might be such an external factor co-determining the patterning outcome of a putative 
reaction-diffusion mechanism underlying microtubule patterning in protoxylem formation. The input of gravity during xylem formation could be relevant since it allows optimisation of the patterning related to local gravity conditions, for example when shoots bend.

Inthischapterwe investigated if gravity is afactorthatis of influence on the positioning and/or the dimensions of secondary cell wall thickenings. By inducible, ectopic activation of the master regulator for protoxylem formation, VND751, we triggered transdifferentiation of Arabidopsis hypocotyl epidermal cells into protoxylem vessel elements. During protoxylem transdifferentiation the microtubule array rearranges into an array with evenly spaced, transverse microtubule bands that template the deposition of secondary cell wall thickenings (chapter $2 \& 3$ ). We conducted a series of experiments in which we ectopically induced VND7 activity and subsequently exposed seedlings to simulated microgravity and hypergravity conditions. Live cell imaging of microtubule reorganization during altered gravity conditions is technically challenging; therefore we used the banded secondary cell wall thickenings as readout for microtubule patterning. The positions of the secondary cell wall thickenings reflect the positioning of cortical microtubules during the experiments and once deposited, the secondary cell wall thickenings are stable and not affected by fixation. We did not observe changes in global secondary cell wall thickening patterning and in the dimensions of secondary cell wall thickenings due to simulated microgravity or hypergravity conditions during protoxylem formation. No significant differences in distance or width of secondary cell wall thickenings can be connected to a specific gravity condition. This indicates that the microtubule reorganization is not sufficiently changed in order to cause disruption of secondary cell wall thickening patterning during protoxylem formation. These results demonstrate that the protoxylem formation is a robust system which is not affected by altered gravity conditions.

\section{Materials and Methods}

\section{Plant material and growth conditions}

Arabidopsis (Arabidopsis thaliana) ecotype Columbia carrying an inducible 35S:VND7-VP16-GR construct ${ }^{51}$ were used for all experiments. Seeds were surface sterilized, stratified for 2 days at $4^{\circ} \mathrm{C}$ in the dark, and grown in petri dishes with $1 \%$ agar containing Hoaglands medium at pH 5.7 (Phyto Technology Laboratories, Kansas - USA). Plates were incubated at $25^{\circ} \mathrm{C}$ with a $16 / 8 \mathrm{~h}$ photoperiod at $45 \mu \mathrm{mol}$ $\mathrm{m}^{-2} \mathrm{~s}^{-1}$. 


\section{Induction of protoxylem formation and sample preparation}

Three day old VND7-seedlings, transgenic Arabidopsis seedlings expressing 35S:VND7-VP16-GR, were transferred to $10 \mu \mathrm{M}$ dexamethasone (DEX) in sterile water. The seedlings incubated on a rotary shaker (110 revolutions per minute) for 1 hour at room temperature. Hereafter, seedlings were transferred to Falcon tubes completely filled with $0.2 \%$ agar supplemented with Hoagland's modified basal salt mixture so that no air bubbles were present in the tube (Phyto Technology Laboratories, Kansas - USA, pH 5.7). Tubes were placed in the RPM or LDC (see below) and exposed to altered gravity conditions for 72 hours in the dark. After 72 hours, the experiment stopped and the VND7-seedlings with ectopically induced VND7 activity were removed from their tubes and fixed in $70 \%$ ethanol in water.

\section{Large Diameter Centrifuge (LDC)}

The hypergravity treatment was applied by placing the samples in a LDC at ESAESTEC (Noordwijk, The Netherlands) ${ }^{104}$. The LDC has a diameter of 8 meters with 4 arms to which gondolas can be attached halfway or at the end. The LDC has swing out gondolas keeping the $\mathrm{g}$ vector perpendicular to the bottom of the gondola. The LDC rotated 30 revolutions per minute. Rotation of the LDC resulted in an acceleration vector of $2 \mathrm{~g}$ or $4 \mathrm{~g}$, depending on the position along the LDC-arm. A rotating gondola located at the centre of the LDC functioned as a $1 \mathrm{~g}$ rotational control. A stationary control was placed in the vicinity of the LDC in the same room.

\section{Random Positioning Machine (RPM) and clinostat}

Microgravity was simulated using a RPM and a clinostat at ESA-ESTEC. The RPM consists of two independent frames that rotate randomly in order to continuously change the direction of the gravity vector ${ }^{105,106}$. The rotation direction and speed of the RPM frames changes randomly with a maximum rotation speed of $\pm 60^{\circ}$ $\mathrm{sec}^{-1}$. For the clinostat experiment we used a second RPM in which we only used one axis rotating at a constant speed of 60 revolutions per minute in a constant rotation direction. The samples are attached in the centre of the frame of both the RPM and the clinostat in such a way that the residual acceleration on the RPM with a maximum distance from the centre of about $7 \mathrm{~cm}$, was not more than $10^{-4} \mathrm{~g}^{106} . \mathrm{In}$ clinostat mode the tubes rotated along their axes.

\section{Microscopy}

Imaging was performed with an Andor spinning disk microscope composed of a Nikon Ti Eclipse body with automated focus correction (PFS3), equipped with 
a iXon ${ }_{3}$ 888-BV monochrome EMCCD camera (Andor) and Yokogawa CSU-X1 spinning disk unit, using a 60x Plan VC objective (NA 1.4). Propidium iodide (SigmaAldrich P4170) was excited with a DPSS $561 \mathrm{~nm} \mathrm{CW} \mathrm{laser} \mathrm{(50mW} \mathrm{Cobolt)}$ at $8 \%$ and emission was detected with a 589-625 nm BP filter (Semrock TR-F607036 filter in Rotr filter wheel). Typical exposure times were $2000 \mathrm{~ms}$ per frame in the z-stacks (using an ASI stage with $150 \mu \mathrm{m}$ z-range).

\section{Data analysis}

Distances between and widths of secondary cell wall thickenings were measured using the open source image processing package FIJI73,79. Distances were measured by creating a line-intensity-plot over the secondary cell wall thickenings at the edge of transdifferentiated cells. The intensity peak of each secondary cell wall thickening was defined as its centre.

\section{Results}

\section{In all altered gravity conditions regular patterns of secondary cell wall thickenings are present}

Initially, we checked if altered gravity conditions prevented the patterned deposition of secondary cell wall thickenings during protoxylem formation. We ectopically induced VND7 activity in VND7-seedlings, transgenic Arabidopsis seedlings expressing 35S:VND7-VP16-GR, and exposed them to different gravitational conditions for 72 hours, sufficient to fully complete the developmental program under normal gravity conditions (chapter 2). As a first assessment, we observed the overall pattern of secondary cell wall thickenings in VND7-seedlings with ectopically induced VDN7 activity which experienced altered gravity conditions. Microgravity was simulated using a clinostat or a RPM, whereas hypergravity was simulated using a LDC to generate $2 \mathrm{~g}$ or $4 \mathrm{~g}$. In all of the altered gravity conditions regular patterned secondary cell wall thickenings are present (Fig. 5.1), demonstrating that secondary cell wall deposition and the ability to contain deposition to specific regions of the cortex are gravity-independent.

\section{Altered gravity conditions did not cause specific changes to the patterned deposition of secondary cell wall thickenings}

We showed that altered gravity conditions did not prevent pattern formation or deposition of secondary cell wall thickenings (Fig. 5.1). We tested if the altered 
gravity treatment caused any obvious defects in secondary cell wall thickening deposition during protoxylem formation. Not all the observed patterns resembled the expected pattern of distinct secondary cell wall thickenings perpendicular to the long cell axis of the transdifferentiated cell (chapter 2). A subset of observed patterns showed secondary cell wall thickenings deviating in width, curvature and/or intermingling branching of secondary cell wall thickenings. The deviation could be excessive, resulting in a pattern bearing a resemblance to the metaxylem rather than the expected protoxylem. Furthermore, a subset showed secondary cell wall thickenings of which only the edges are stained, while the area in between two edges is lacking staining. As an additional assessment, we categorised the observed pattern and compared the distribution of patterns for the different gravity treatments (Fig. 5.1 AB). The observed patterns were segregated in three categories with 'type-1' for patterns resembling the expected protoxylem pattern (chapter 2), 'type-2' for patterns with fully stained secondary cell wall thickenings which deviated in width, curvature and/or branching and 'type-3' for patterns with secondary cell wall thickenings of which only the edges were stained (Fig. 5.1C). Type- 1 and type-3 patterns are observed in all altered gravity conditions. In simulated microgravity, type-2 patterns are observed in the RPM control treatment (2 out of 31 cells), in the clinostat 2D treatment (1/31 cells) and in the RPM 3D treatment (7/38 cells) (Fig 5.1A). In simulated hypergravity, type-2 patterns occur in the rotating control at $1 \mathrm{~g}(3 / 37$ cells) and in the $2 \mathrm{~g}$ treatment ( $2 / 40$ cells), however type- 2 patterns are absent in the stationary control and the $4 \mathrm{~g}$ treatment (Fig 5.1B). Although some cells have deviant secondary cell wall thickenings (type2 \& type-3) from the typical patterning in protoxylem vessel elements (type-1), these deviant patterns are not specifically correlated to specific altered gravity conditions. In contrast, the deviant patterns occur randomly distributed over the treatments. Therefore, altered gravity conditions did not cause significant defects to the patterned secondary cell wall thickening deposition in a specific way. 
A

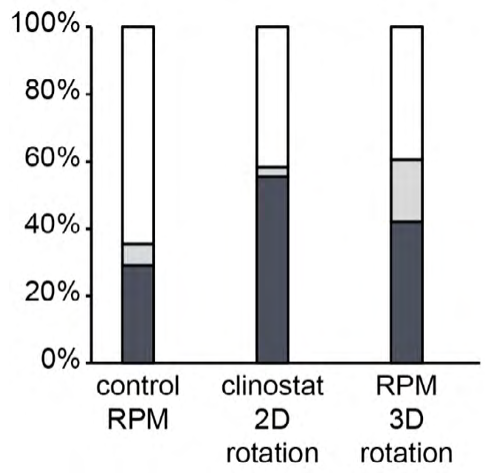

B

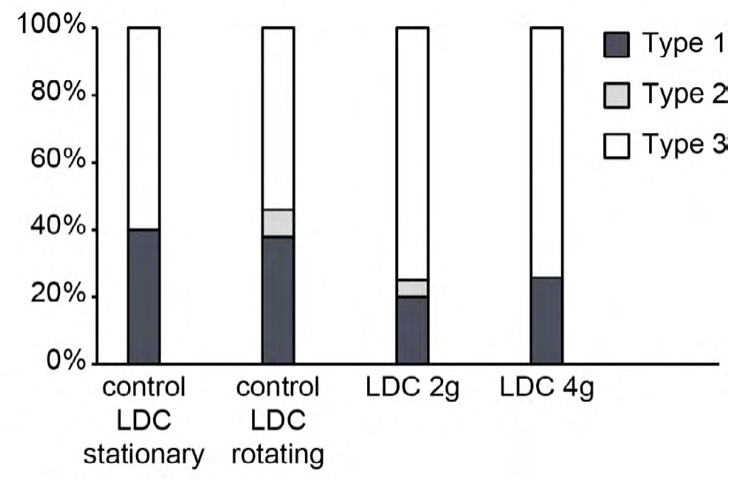

C Type 1

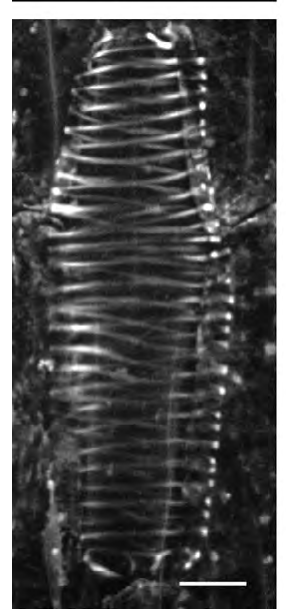

Type 2

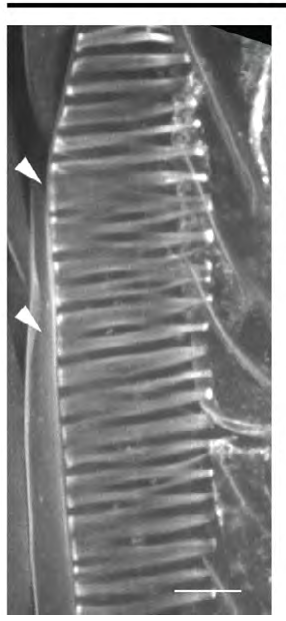

Type 3

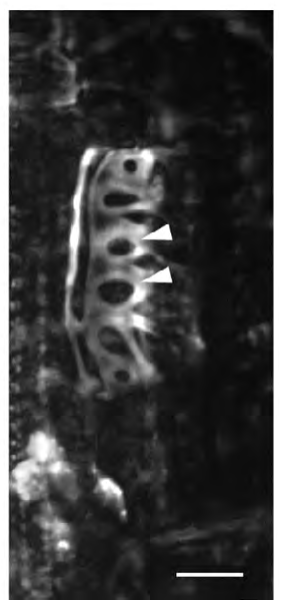

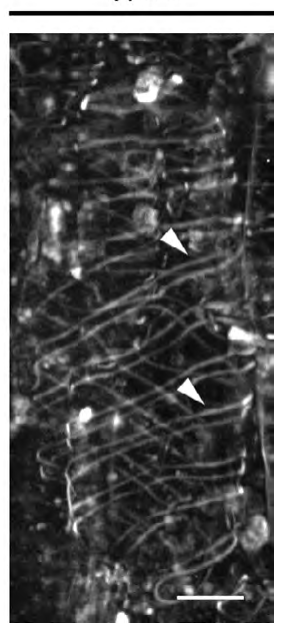

Figure 5.1 | Typically observed secondary cell wall thickening patterns and their distribution in transdifferentiating hypocotyl cells of VND7-seedlings with ectopically induced VND7 activity in altered gravity conditions. Besides regular secondary cell wall thickenings, we observed cells with deviations in secondary cell wall thickening patterning. Deviations in width, curvature and branching of secondary cell wall thickenings were observed, as well as staining of only the edges of secondary cell wall thickenings. (A-C) Observed patterns were categorised as 'type-1' for regular protoxylem patterns, 'type-2' for deviations in shape and position of secondary cell wall thickenings and 'type-3' for specific staining of the edges of secondary cell wall thickenings. In all altered gravity conditions type- 1 and type-3 patterns are observed. (A) In simulated microgravity treatment more type-2 patterns are present in the RPM 3D treatment $(18.4 \%)$ compared to the control treatment (6.5\%). (B) In the enhanced gravity treatment (LDC) the only type-2 patterns are observed in the rotating LDC control (8.1\%) and the LDC $2 \mathrm{~g}$ treatment (5\%). (C) Example of the observed types of secondary cell wall thickening patterns. Secondary cell wall thickenings were visualized by staining with $10 \mu \mathrm{M}$ propidium iodide. White arrows in type-2 patterns indicate secondary cell wall thickenings with an increased width when compared to type-1 secondary cell wall thickenings, and non-transverse secondary cell wall thickenings which are not present in type- 1 patterns. White arrows in the type- 3 image indicate secondary cell wall thickenings of which only the edges have been stained. Scale bars $=10 \mu \mathrm{m}$. 


\section{Positioning and width of secondary cell wall thickenings are not affected by exposure to altered gravity conditions}

The initial assessments show that the formation of secondary cell wall thickenings is not inhibited by the altered gravity conditions, and although occasionally deviant secondary cell wall thickening patterns were observed, they do not correlate with altered gravity conditions. To test if the effect of gravity on microtubule patterning and cell wall deposition has more subtle effects on the formation of secondary cell wall thickenings, one could quantify parameters of secondary cell wall thickenings such as spacing, width, thickness, curvature, branching and cellulose content. Here we quantified the spacing and width of secondary cell wall thickenings that were deposited under altered gravity conditions. We performed the analyses on cells of the type-1 category to exclude cells with strongly deviating patterns that increase the variation in measurements within individual groups. Measurements were performed on at least 3 independent seedlings and at least 8 cells and totalling at least 97 measurements of each parameter per treatment (Table 5.1). No significant differences were found between control treatments and altered gravity conditions for both the width and the spacing of secondary cell wall thickenings (Fig 5.2). VND7-seedlings with ectopically induced VND7 activity in the rotational control of the LDC did show a significant increase in width of secondary cell wall thickenings compared to the stationary control (Fig. 5.2C). Note that this is a control treatment in which gravity conditions were not altered and therefore the significance of this difference is limited. Since the average width and distances in cells with type- 1 patterns did not differ significantly between control and altered gravity conditions, gravity is not a factor that is involved in positioning and determination of the width of secondary cell wall thickenings.

Table 5.1 | The number of plants, cells and measurements per treatment to generate the data represented in Figure 5.2.

\begin{tabular}{|c|c|c|c|c|c|c|c|}
\hline & $\begin{array}{c}\text { control } \\
\text { RPM }\end{array}$ & $\begin{array}{c}\text { clinostat } \\
\text { 2D }\end{array}$ & $\begin{array}{c}\text { RPM } \\
\text { 3D }\end{array}$ & $\begin{array}{l}\text { control LDC } \\
\text { (stationary) }\end{array}$ & $\begin{array}{c}\text { control LDC } \\
\text { (rotating) }\end{array}$ & LDC $2 g$ & LDC $4 g$ \\
\hline \# measured plants & 5 & 5 & 5 & 4 & 5 & 5 & 3 \\
\hline \# measured cells & 9 & 20 & 16 & 10 & 14 & 8 & 9 \\
\hline \# measured widths & 98 & 313 & 249 & 153 & 221 & 139 & 127 \\
\hline \# measured distances & 97 & 249 & 160 & 113 & 195 & 100 & 102 \\
\hline
\end{tabular}



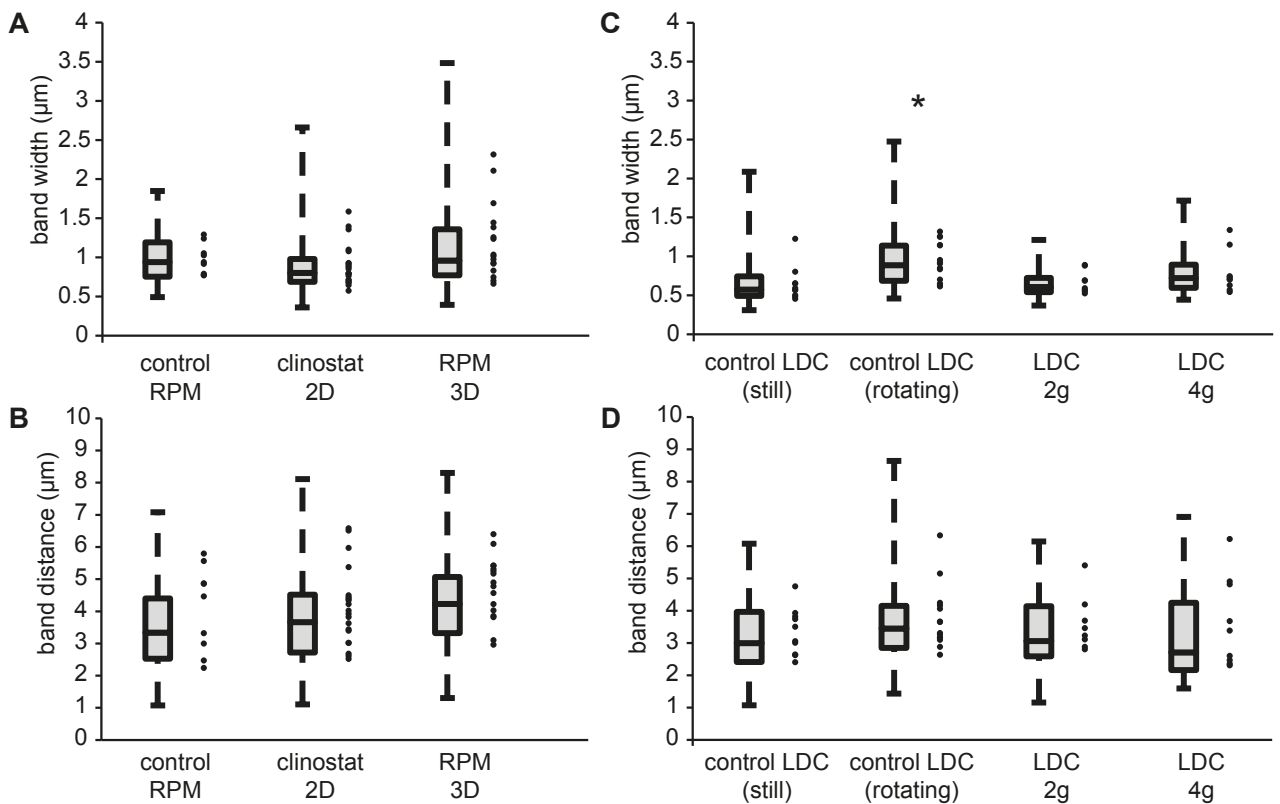

Figure 5.2 | Quantification of widths and distances of secondary cell wall thickenings in hypocotyl cells after VND7-induced transdifferentiation under altered gravity conditions. The boxplot represents the data of all separate measurements per treatment. The boxplot graphs indicate the $1^{\text {st }}$ quartile, the median and $3^{\text {rd }}$ quartile of all measurements per treatment in type1 cells. The whiskers of the boxplot indicate the minimum and maximum observed values. The scatter plots represent the individual averages per hypocotyl cell. Significant differences between treatments is tested using the Mann-Whitney test since data is not normally distributed. The asterisk indicates a $p$-value $\leq 0.05$.

\section{Discussion}

In this chapter we have used the patterning of secondary cell wall thickenings as a proxy to study whether gravity is a factor in controlling the reorganization of cortical microtubules that template these secondary cell wall thickenings. The master regulator for protoxylem formation, VND7 ${ }^{45}$, was induced ectopically and the patterning, width and distance between the resulting secondary cell wall thickenings in hypocotyl epidermal cells were analysed. We show that patterned deposition of secondary cell wall thickenings is not disrupted under simulated microgravity or hypergravity conditions (Fig. 5.1). In addition, there are no significant differences of average band width or average distance of secondary cell wall thickenings under these conditions (Fig. 5.2). From these results, we conclude that the microtubule reorganization that occurs before the deposition of secondary cell wall thickenings is sufficiently insensitive to altered gravity conditions that the deposition of secondary cell wall thickenings is not affected. 
The experiments to test if gravity is able to influence microtubule patterning during protoxylem formation, resulted in three different pattern types of secondary cell wall thickenings (Fig. 5.1). The type-1 pattern resembled transdifferentiated protoxylem as observed in VND7-seedlings with ectopically induced VND7 activity (chapter 2), whereas type-2 and type-3 patterns were deviant. Type-2 and type-3 patterns do not occur consistently with a particular gravity condition. Therefore, the altered gravity is unlikely to be the cause of the deviant patterns. The occurrence of deviant patterns in control treatments suggests that the experimental setup might cause these patterns. During the experiments described in this chapter, after induction of VND7 expression, seedlings were kept in the dark in a tube with limited gas exchange to enable mounting the samples to the RPM and the LDC. These experimental conditions differed from the experimental setup used for experiments in chapter $2 \& 3$. Preceding the RPM and LDC experiments, trial experiments were conducted at Wageningen University which indicated that protoxylem transdifferentiation was not affected by the used experimental setup. However, this characterization was not as extensive as in chapter 2. Furthermore, mechanical vibration might cause deviations in patterned deposition of secondary cell wall thickenings, as almost all samples showed deviant type-2 patterns, except for the stationary control and the LDC $4 \mathrm{~g}$ treatment. The experimental conditions in the ESTEC facilities where the RPM and the LDC were located may have been less alike than anticipated when compared to the growth facilities used for the experiments in chapter $2 \& 3$. Nonetheless, type-1 patterns were present in all altered gravity conditions, demonstrating that the altered gravity did not prevent microtubule reorganization and patterned deposition of secondary cell wall thickenings. Seedlings were in tubes with $0.2 \%$ agar, and thus there was no preferential direction of gravity in the LDC treatments. Potentially, less type-1 patterns will be observed if seedlings do experience a constant direction under hypergravity conditions.

The finding that gravity does affect the microtubule array in wall-less protoplasts ${ }^{64,65}$ but not in tobacco BY-2 cells with an intact cell wall, caused Sieberer et al. (2009) to suggest that information between the microtubule array and the cell wall is bidirectional ${ }^{101}$. This idea has been presented before based on theoretical and experimental data ${ }^{68}$. Watanabe et al. (2015) provided experimental results showing that oryzalin treatment of transdifferentiating cells in VND7-seedlings with ectopically induced VND7 activity did not affect the velocity of cellulose synthase complexes responsible for cellulose microfibril deposition in secondary cell wall thickenings, and removal of cellulose synthase complexes via DCB treatment did not affect the typical banded microtubule pattern ${ }^{52}$. This suggests that information flow from the cell wall to the microtubule array is not crucial for the patterning of secondary cell wall thickenings during protoxylem formation. 
Since we do not observe differences in width and distance of secondary cell wall thickenings, this supports the finding that if altered gravity conditions are able to affect the microtubule organization ${ }^{64,65}$, this effect can be overruled when a cell wall is present ${ }^{101}$.

The theory of reaction-diffusion systems and bifurcation theory provide a mechanism how gravity could affect the self-organization of the microtubule array. This raises some questions regarding pattern formation of the cortical microtubule array and localized deposition of secondary cell wall thickenings during protoxylem formation. Is gravity able to affect microtubule array reorganization during protoxylem formation? Is gravity providing a directional bias to microtubule array reorganization? Is this effect overruled by the cell wall or other cellular pathways? It could be that microtubule array organization is affected by gravity but that correct patterning of secondary cell wall thickenings is crucial and the effect is counteracted by positional information provided by the cell. To investigate these possibilities one would need to study the microtubule dynamics during microtubule array reorganization, like we did in chapter 3, during altered gravity treatment.

We show that altered gravity conditions does not change patterned deposition of secondary cell wall thickenings in transdifferentiating VND7-seedlings with ectopically induced VND7 activity. This indicates that gravity does not sufficiently affect microtubule patterning to change patterned deposition of SCTWs during protoxylem formation. This is a favourable result considering that viable plants with normal plant growth are necessary in bioregenerative life support system to facilitate long-term space missions in the future ${ }^{107}$. It is not likely that microgravity in space will affect protoxylem formation and thereby reduce plant development resulting in unviable plants. 


\section{Chapter}

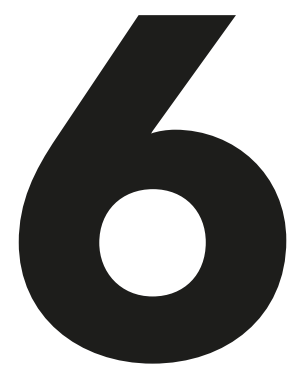

General discussion 
The world population showed a tremendous growth in the past 50 years, and the United Nations predicts that by the year 2050 inhabitants on this earth surpasses 9 billion. This population growth has been made possible by the transition towards farming civilizations and food production has gained a boost via the green revolution. New challenges are faced by humanity, science in general and plant science specifically due to this projected population growth. Applied sciences, such as plant breeding, can aid in increasing the world food production. Fundamental plant science, such as described in this thesis, will not directly provide answers to the challenges faced in the future. But fundamental science does add to the current knowledge and thereby provides a framework in which the applied sciences can navigate towards new solutions. The worth of a scientific finding can therefore only be judged in retrospection.

Plant cells form the basic building block of each plant. To understand plants, it is thus crucial to understand how the plant cell functions. Plant cell biology improves understanding of plant biology in general. An interesting area of investigation is the surface of a plant cell, consisting of the plasma membrane and the cell wall. Together they form the interface at which the plant cell interacts with its surroundings. Plants are able to sense their environments and react on it by adjusting cell physiology and cell growth. An example of this is gravitropism, by which a plant is able to sense the gravity direction and adjusts the growth direction. Adjusting the growth direction of a plant can be achieved by determining the plane of cell division and by altering the cell wall properties of new cells. The cell wall is the nexus of plant life.

In this thesis, we have studied the temporal and spatial aspects of patterned deposition of secondary cell wall thickenings during protoxylem formation. To understand how banded cell wall thickenings are positioned during protoxylem differentiation, firstly, one should address the question how plant cells can form a pattern, and next, how this pattern is translated into deposition of cell wall material according to a patterned template. As shown in the previous chapters, the cortical microtubule array determines when and where cell wall material is deposited during protoxylem formation. The cortical microtubule array consist of a network of filamentous protein polymers attached to the cytoplasmic side of the plasma membrane ${ }^{31}$. In this discussion, I will combine current knowledge concerning the function of the cortical microtubule array and cell wall deposition with the data presented in this thesis. Firstly, I will discuss the cell wall deposition machinery, then, how this machinery is regulated, and finally, how patterns of secondary cell wall thickenings are formed during protoxylem formation. 


\section{Regulation of primary cell wall deposition by plant cells}

Plant cell walls consist cellulose microfibrils that are embedded in a matrix of pectin and hemi-cellulose ${ }^{2}$. Cellulose microfibrils are thought of as the loadbearing components in a cell wall, resembling steel cables in reinforced concrete $^{3,4}$. The orientation of the cellulose microfibrils in cell walls is thought to determine plant cell elongation direction and thereby cell shape ${ }^{108,109}$. Cellulose microfibrils are synthesised by protein complexes, cellulose synthase complexes, which are located in the plasma membrane ${ }^{110,111}$. Cellulose synthase complexes contain cellulose synthase (CesA) proteins which join together cytoplasmic UDPglucose molecules to form long polymers which are deposited between plasma membrane and the existing cell wall ${ }^{42}$. The movement and insertion of cellulose synthase complexes in the plasma membrane is guided by the microtubule network attached to the cytoplasmic side of the plasma membrane during primary cell wall deposition ${ }^{38-40}$. The mechanism of the interaction between the microtubule array and cellulose synthase complexes is further elucidated by the discovery of the suggested scaffold protein POM2/CSI1 between cellulose synthase complexes and microtubules ${ }^{112}$. Besides guiding cellulose microfibril synthesis, the microtubule cytoskeleton is also involved in delivery of cell wall matrix material ${ }^{113,114}$. In phototropism and gravitropism, the attribute of plants to grow towards a light source or a gravity factor respectively, the microtubule array is reoriented upon a change in light source or gravity factor ${ }^{44,115-117}$. Altogether, these findings indicate that the microtubule array templates the location and direction of cellulose deposition during the synthesis of primary cell walls.

\section{Cortical microtubules guide cellulose deposition in localized secondary cell wall thickenings during xylem formation}

Cellulose synthase complexes responsible for cellulose microfibril synthesis in Arabidopsis (Arabidopsis thaliana) is a protein complex of the cellulose synthases CesA4, -7 and $-8^{9}$. Mutants in these secondary CesAs result in dwarfed Arabidopsis plants, caused by collapsed vessel elements indicating the importance of the secondary cell wall thickenings ${ }^{9-12}$. Tracheary elements are characterized by typical patterned secondary cell wall thickenings. To generate these patterns it is not sufficient to reorient the direction of cellulose microfibrils as it is for phototropism or gravitropism. Besides orientation, also the position of the cellulose microfibrils needs to be spatially regulated to create secondary cell wall thickenings in bands. The microtubule array is a likely candidate to facilitate this pattern formation since insertion and movement of cellulose synthase complexes is occurring alongside cortical microtubules ${ }^{38-40}$. Furthermore, microtubules are dynamic and contain self- 
organization properties allowing microtubule arrays to reorganize ${ }^{35}$. Microtubules have been shown to form high density bands of microtubules underneath developing secondary cell wall thickenings via electron microscopy ${ }^{118}$ and light microscopy techniques ${ }^{60}$. Microtubule drugs are known to affect the deposition of secondary cell wall thickenings in tracheary elements $47,49,70,72,119$. Fluorescent tagged CesA7 was used to track movement of cellulose synthase complexes, confirming the association of cellulose synthase complexes with microtubules during deposition of localized secondary cell wall thickenings in Arabidopsis ${ }^{52}$. Thus, to understand patterned deposition of cell wall material during xylem formation, we need to understand the organization of the microtubule array during differentiation of xylem cells. How is it possible that the microtubules that template the deposition of secondary cell wall thickenings get organized in a regular pattern of microtubule bands during protoxylem formation?

\section{The microtubule array as a self-organizing system}

Microtubules are, as the name indicates, small and tubular protein polymers with an approximate diameter of $24 \mathrm{~nm}$ and are just like in all eukaryotes, part of the cytoskeleton ${ }^{24,25}$. In interphase plant cells, microtubules are attached to the plasma membrane forming a two-dimensional cortical microtubule array ${ }^{31}$. Early electron microscopy studies showed that cortical microtubules underlie cell wall thickenings in developing xylem cells $s^{31,70,119}$. Live cell fluorescence microscopy studies showed that during protoxylem formation the microtubule array forms a regular pattern with high density microtubule bands separated by low density microtubule gaps ${ }^{60,72}$ (chapter 2). It remains unclear how the microtubule array reorganizes into this regular banded pattern, while it is restricted to a two-dimensional plane due to the attachment to the plasma membrane. Microtubules are dynamic as microtubules grow and shrink continuously. The switching between growth and shrinkage and vice versa of microtubules is defined as respectively a catastrophe and a rescue. The combination of these dynamics result in a phenomenon termed dynamic instability, in which the microtubule stochastically changes from a growing to a shrinking state. The angle of a growing microtubule with an existing microtubule determines the chance of co-alignment versus depolymerisation ${ }^{34}$. These local interactions between individual microtubules affect the global microtubule array organization $28,34,58,87$.

Microtubule associated proteins (MAPs) are able to control, change and direct the dynamic instability for example by affecting the nucleation of microtubules, the growth and shrinkage speeds, and the catastrophe and rescue rates ${ }^{35}$. KATANIN is a MAP able to sever microtubules, another MAP-function, and is required in blue light induced reorientation of the microtubule array involved in phototropism in 
Arabidopsis ${ }^{44}$. In the MAP mutants spr1, eb1 and claps1-1, reorientation of the array occurs but is delayed ${ }^{120}$, highlighting the importance of MAPs in determining the self-organization properties of the microtubule array. Thus, microtubule arrays can reorganize over time in plants cells using dynamic instability and MAP activity. To understand patterned secondary cell wall deposition during protoxylem formation it is required to understand changes in dynamic instability parameters and activity of MAPs. This will provide insight in how the regular banded pattern of microtubule bands and gaps emerges during protoxylem formation.

\section{Molecular components involved in metaxylem patterning}

Some molecular components involved in metaxylem pattern formation have been identified. AtKinesin13A and MIDD1 are important MAPs during metaxylem formation ${ }^{54,55}$. AtKinesin $13 A$ and MIDD1 remove cortical microtubules, respectively by depolymerising microtubules and supressing rescues, in xylem cells differentiating into a cell with a pitted pattern from the future pit regions $s^{54,55}$. The deposition of secondary cell wall material is thereby excluded from the regions with a low microtubule density, resulting in the characteristic pitted pattern. The activity of these MAPs explains how microtubules are depleted from membrane areas underlying future pits. Nonetheless, the question remains how the positioning of these pits is controlled? To answer this question, the upstream proteins that control the activity of MIDD1/AtKinesin13A based microtubule depletion needs to be taken into account. GTPases are suitable candidates known to be involved in plant cell polarity regulation and their ability to interact with the cytoskeleton ${ }^{121}$. Local self-sustaining feedback loops between GTPases and the cytoskeleton regulate cell polarity and determine vesicular trafficking enabling cell morphogenesis ${ }^{121}$. This mechanism is elegantly demonstrated in the morphogenesis of pavement cells in plants with a jigsaw-puzzle like appearance. Plasma membrane localized active ROP2 interacts with RIC4 to promote F-actin and thereby cell outgrowth, whereas ROP2-RIC1 promotes ordering of the microtubule array suppressing local cell growth ${ }^{122}$.

In metaxylem formation the pattern of the MAPs AtKinesin13A and MIDD1 are controlled by the plasma membrane bound GTPase ROP11 which is activated and deactivated by ROPGAP3 and ROPGEF4, respectively ${ }^{53}$. A reaction-diffusion mechanism has been suggested to underlie the pattern formation of ROP11, ROPGAP3 and ROPGEF4 ${ }^{53}$. Reaction-diffusion mechanisms are based on reacting components coupled by different diffusion speeds enabling pattern establishment ${ }^{20}$. Cortical microtubules have been shown to be able to act as a barrier to membrane bound ROP11-MIDD1 complexes in leaf epidermal 
cells of Nicotiana benthamiana ${ }^{53}$. It is unclear if and how this barrier function of microtubules affects patterned deposition of secondary cell walls during xylem formation in Arabidopsis. If microtubules do provide feedback to ROP11MIDD1 complexes, the length distribution of microtubules could be important in metaxylem formation. For the length distribution to change, the microtubule dynamic parameters should change. We showed that microtubule dynamics do change during protoxylem formation coinciding with the emergence of microtubule gaps and bands (chapter 3 ). Assuming protoxylem and metaxylem pattern formation follow similar mechanisms, it would be interesting to analyse microtubule dynamic parameters during metaxylem formation.

\section{Putative molecular components involved in protoxylem patterning}

Since microtubule patterning is crucial in protoxylem formation (chapter 2) just as it is in metaxylem formation, the patterning mechanism might be conserved. In metaxylem formation AtKinesin13A and MIDD1 are responsible for removal of microtubules from future pit regions. ROPGEF4, ROPGAP3 and ROP11 are responsible for positioning MIDD1 to local domains enabling the characteristic pitted pattern. It would be tempting to hypothesize that similar molecular components are involved to remove microtubules in the microtubule gap area during protoxylem formation. However, a ropgef4 mutant did not alter protoxylem patterns ${ }^{53}$, suggesting alternative pathways than the ROP11-MIDD1 pathway might function to regulate protoxylem patterning. Similar to pit formation in metaxylem vessel elements, microtubule gaps could emerge by removal of cortical microtubules in protoxylem vessel elements. An alternative to MIDD1AtKinesin13A dependent removal of microtubules could be KATANIN, a MAP known to sever microtubules ${ }^{123}$ and critical for reorganization of microtubule arrays upon blue light stimulation ${ }^{44}$. Instead of focussing on microtubule gap emergence, gaps could emerge passively if microtubules get stabilized in microtubule bands. Bundling of microtubules might be a mechanism to promote microtubule presence in the microtubule bands. From in vitro Zinnia elegans cell culture studies it is known that ZeMAP65-1 promotes microtubule bundling during xylem differentiation ${ }^{46}$. We observed microtubule bands in which not all microtubules are tightly bundled but nonetheless the microtubules appeared to be restricted to the microtubule band area. Pesquet et al. (2010) showed that a specific MAP, AtMAP70, may function in defining the boundaries of microtubule bands in Arabidopsis cells undergoing xylem formation in a hormonal induced cell culture system ${ }^{49}$. The abundance of spiral patterns increased due to overexpression of AtMAP70, whereas RNAi silencing increased pitted patterns in induced cells ${ }^{49}$. This suggest that the abundance of MAP70, in combination with MAP65 and 
MIDD1, may determine the type of patterning: low abundance of MAP70 resulting in metaxylem patterns and high abundance in protoxylem patterns ${ }^{13}$.

Besides bundling, nucleation could be an important process to study in order to know how the microtubule array changes. New microtubules can nucleate from nucleation complexes ${ }^{26}$, which could be regulated to establish and maintain a regular patterned microtubule array with microtubule bands. Both the location of new microtubule nucleation and the nucleation angle is important for the microtubule array organization and is controlled by nucleation complexes ${ }^{124,125}$. GCP2 is part of the nucleation complex and live cell imaging revealed that GCP2 based microtubule nucleation predominantly occurs on existing microtubules ${ }^{125}$. If nucleation complexes are localized predominantly to (future) microtubule band areas, this will establish microtubule growth in the band areas and reduce nucleation in the microtubule gaps. Once microtubule bands are formed, these areas with a high density of microtubules could recruit new nucleation complexes, and thereby provide a positive feedback loop to maintain the presence of the microtubule band. This putative reinforcement mechanism could be tested experimentally by studying nucleation dynamics during protoxylem formation. An experimental approach to assess nucleation is to use fluorescently tagged end binding proteins such as EB1 ${ }^{126,127}$ and nucleation complexes ${ }^{128}$. When applied during xylem transdifferentiation, these probes would allow for quantification of the location and angles of newly formed microtubules in microtubule gaps and bands. A reinforcement mechanism of nucleation cannot be the sole mechanism as this could lead to hyper accumulation of microtubules in one band. The microtubule bands observed during protoxylem formation are not required for cell wall deposition, but do target the cell wall deposition machinery to the correct location (chapter 2; Fig. 2.3). The molecular regulatory pathways in protoxylem patterning remain elusive. To assess similarity between proto- and metaxylem patterning, the molecular components discusses above needs to be visualized during protoxylem formation. To asses if similar mechanisms are used for microtubule pattern formation in protoxylem and metaxylem vessel elements, the microtubule dynamic parameters needs to be quantified in metaxylem formation similarly as we provided in this thesis for protoxylem formation (chapter 3).

\section{Patterned cell wall thickenings as a physical output of self-organized microtubule arrays}

Periodic patterned deposition of cell wall material forming rings and spirals, as observed in protoxylem formation (chapter $2 \& 3$ ), requires both that a pattern emerges and that this pattern is translated into a physical output. Let us now look 
in more detail into how a pattern can emerge via a reaction-diffusion mechanism (as mentioned before), in which multiple components react and are coupled by different diffusion speeds ${ }^{20}$. Typical for reaction-diffusion system is that stable periodic patterns can emerge in out-of-equilibrium systems which can have bifurcation points, points in which a small difference can determine the final state of the system ${ }^{61,62}$. Reaction-diffusion mechanisms are proposed to govern pattern formation in biological systems at different scales, from ecosystems to cells. Recently, self-organization properties of vegetation and termites on a small scale were coupled to explain patterned vegetation in which patches bare of vegetation are evenly spaced over a large scale ${ }^{16}$. The Turing reaction-diffusion model can generate biological patterns similar as observed in nature, for example in fish skin patterns and sea shell patterns ${ }^{21}$. At cell level, self-organizing properties determine cell polarity, such as in the MIN-system of single celled bacteria ${ }^{17,102}$. The influence of a weak external field change on microtubule self-organization properties in vitro suggest that in this system a bifurcation point exists depending on gravity ${ }^{63}$. A reaction-diffusion mechanism has been proposed to be important for metaxylem patterning ${ }^{53}$. Both in protoxylem and metaxylem formation patterns emerge at the cell level, which could depend on a common mechanism, such as a reaction-diffusion mechanism. Secondary cell wall thickenings in protoxylem vessel elements are typically positioned perpendicular to the gravity vector, which could be a direct result of the gravity field or indirect via the plant cell shape. Therefore the question arises if protoxylem patterning is based on a reaction-diffusion mechanism and if gravity is able to direct the positioning of microtubule bands which guide secondary cell wall deposition. Experiments in which protoplasts, plant cells without cell walls, are exposed to altered gravity conditions, show that the microtubule array is affected ${ }^{64,65}$. On the other hand, in BY-2 tissue culture cells these gravity effects have not been observed ${ }^{101}$. This suggests that the cell wall, not present in protoplast but present in BY-2 cells, could negate a gravity induced effect on the microtubule organization. To assess the effect of gravity on microtubule array self-organization when a cell wall is present, it might be needed to study a non-equilibrium system (chapter 5 ), such as the reorganization of the microtubule array during protoxylem formation (chapter 2 \& 3 ). The patterns of deposited secondary cell wall thickenings in protoxylem vessel elements are known in normal gravity conditions (chapter 2). Patterned secondary cell wall thickenings of protoxylem vessel elements can be regarded as physical output of the microtubule reorganization which occurred during protoxylem differentiation. We used the patterned secondary cell wall thickenings as a proxy for microtubule pattern formation since we know that the microtubule pattern template the patterned secondary cell wall thickenings (chapter 2), and it is difficult to assess microtubule dynamics during protoxylem formation in altered gravity 
treatment conditions. Gravity might be able to affect the self-organization of the microtubule array temporally or spatially. We have shown that altered gravity does not spatially affect patterned secondary cell wall deposition indication that the microtubule reorganization is sufficiently insensitive to altered gravity conditions during protoxylem formation (chapter 5). Temporal differences in microtubule reorganization could occur in altered gravity conditions, but these differences are not necessarily translated to the final secondary cell wall pattern.

\section{Benefits and drawbacks of experimental methods to study xylem formation in plants}

Differentiation of tracheary elements in plants occurs in the central stele covered by epidermal and cortex cells in a sequential manner. This gives two problems to study the xylem differentiation process in planta. First, live cell imaging by microscopy is difficult as this requires light to reach the cells of study and to return light carrying information. Light transfer is hampered by epidermal and cortex cells to reach differentiating tracheary elements. Second, each tracheary element is at a different developmental stage in planta which makes it difficult to compare differentiation progress within and between plants in the absence of suitable markers for sequential developmental stages. Microtubules have been visualized in endogenous xylem cells and shown to form bands underlying developing secondary cell wall thickenings $s^{60}$, but the image quality is not sufficient to assess dynamics on a single microtubule level. The discovery of transdifferentiating cell cultures, triggering non-xylem cells to develop into xylem like cells, has greatly helped to identify cellular processes involved in xylem formation. The benefits are that these transdifferentiation cells cultures can be synchronised and are well suitable for analysis by microarrays ${ }^{45,48}$, proteomics ${ }^{50}$ and experimental cell biology $46,47,49,129$. Microarray analysis followed up by overexpression experiments revealed the master regulators for proto- and metaxylem formation, respectively VND7 and VND6 ${ }^{45}$. These master regulators were coupled with an inducible expression system enabling ectopic induction of xylem transdifferentiation in tobacco BY-2 cells, Arabidopsis plants and poplar trees ${ }^{51,55}$. This proved to be a powerful tool to advance the study of xylem formation both via microarray analysis ${ }^{51,81,130}$ and experimental cell biology ${ }^{54-56,131,132}$. In this thesis, inducible ectopic expression of VND7 is used as an experimental system to study functional properties of the microtubule array which templates the patterned deposition of cell wall material in developing protoxylem vessel elements. Although the inducible VND7-system is a powerful tool to study protoxylem formation, it remains an artificial system which awaits confirmation in endogenous protoxylem formation. 


\section{Self-organization of the microtubule array during protoxylem formation}

Which self-organizing microtubule properties should be present to facilitate patterning of the microtubule array? Some molecular components involved in metaxylem patterning have been identified $46,49,53-55$, but in depth microtubule dynamics have not been assessed. Studying microtubule dynamics improved understanding how a cortical microtubule array assembles in plant cells ${ }^{34,128}$. These works shows that insight in microtubule dynamics is key to understand the behaviour of a microtubule array. The work presented in this thesis established a basic understanding in what changes functionally at the level of the microtubule array over time as the microtubule array obtains the typical banded organization that templates the deposition of secondary cell wall thickenings. In a quantitative manner we analysed microtubule dynamics at the single microtubule level (chapter 3). The strength of this approach is that we studied patterning both in the spatial and the temporal domain. The weakness is that we were not able to experimentally connect the observed functional requirements for pattern formation change to specific MAPs or other molecular components responsible for patterning such as ROPs. To facilitate patterning of the microtubule array during protoxylem formation we now can conclude that the self-organization properties of the microtubule array together with molecular components should at least be able to (1) order the microtubule array transversely, (2) spatially destabilize and stabilize microtubules to form respectively microtubule gaps and bands, (3) maintain the established microtubule pattern while (4) allowing for remodelling of the pattern by repositioning and merging microtubule bands if needed. Embarking on a route towards a systems biology methodology, we used a computational approach to probe this second functional insight further. Both our experimental and computational results can be combined to guide the search for molecular components regulating pattern formation of the microtubule array during xylem formation.

\section{Reaction-diffusion mechanism of protoxylem patterns}

The four self-organization properties for protoxylem pattern formation mentioned above may be facilitated by a reaction-diffusion mechanism. We show that microtubule bands are able to merge and emerge over time (chapter 3; Fig. 3.2B), showing that remodelling of the microtubule array pattern does occur even after an initial pattern has been generated. This suggests that there is an optimal spacing for microtubule bands which could be regulated by an underlying reactiondiffusion mechanism that is responsible for the initial pattern formation and allows for adjustment and/or fine-tuning during a later phase. These observations suggest that a patterning mechanism is in place that provides information about 
the distance between bands, the thickness of bands and the angle of bands. This patterning mechanism is able to maintain similar parameters over the long axis of cells. This is consistent with a reaction-diffusion patterning mechanism. Nonetheless, my results do not prove the existence of a reaction-diffusion mechanism, as this would require the identification of molecular components in protoxylem formation, characterization of these components by mutant studies and simulation studies that demonstrate that the identified components are sufficient for the observed pattern generation. Some molecular components have been identified that are involved in pattern formation of pits during metaxylem formation ${ }^{53-55}$, but the molecular components involved in pattern formation of bands and gaps in protoxylem remain to be identified. Even though no significant effect of a ROPGEF4 knockout or the expression of a constitutive active ROP11 on localised deposition of secondary cell wall thickenings in protoxylem was observed $^{53}$, future experiments should test if the ROP11-MIDD1 pathway is involved in pattern formation of the microtubule array during protoxylem formation. Expression profiling using publically available expression data (BAR UToronto) shows that ROP7 expression is specifically upregulated during protoxylem formation ${ }^{82}$. I performed an initial screen for phenotypes in a ROP7 knockout. Unfortunately, rop7 lines did not yield in protoxylem patterning phenotypes at the level of secondary cell wall thickenings in Arabidopsis. A possible explanation for the lack of defects in rop7 lines is that there might be redundancy between ROPs during protoxylem differentiation.

The positioning of the secondary cell wall thickenings in the metaxylem is regulated by cortical microtubules ${ }^{53-55}$. Most likely regulation of the positioning of the secondary cell wall thickenings in protoxylem formation is regulated similarly as in metaxylem, thus also via cortical microtubules. Our results point into a similar direction as we have shown that microtubule reorganization precedes deposition of secondary cell wall thickenings (chapter 2). Therefore it is more likely that a reaction-diffusion mechanism patterns the microtubule array than secondary cell wall deposition directly. Molecular components that are part of a reaction-diffusion mechanism to form a pattern could recruit MAPs which affect the microtubule dynamics and thereby pattern the microtubule array. Microtubules could recruit MAPs affecting themselves, thereby providing the required feedback loops for a reaction-diffusion mechanism. We have shown, via microtubule depolymerizing experiments with oryzalin (chapter 2), that cortical microtubules are essential or for initial pattern formation, or for the translation of an existing pattern to patterned deposition of secondary cell wall thickenings. In addition, the earlier onset of secondary cell wall deposition upon microtubule stabilization by taxol treatment suggests that the dynamic properties of cortical microtubules are important for 
pattern formation. The existing knowledge about CesA complex insertion and guidance along cortical microtubules suggests that the cortical microtubules are important for the translation of a pattern to localised deposition of secondary cell wall thickenings.

Our data does not exclude that a similar molecular mechanism is operational both in the proto- and metaxylem, in protoxylem putatively with other proteins than MIDD1 and AtKinesin13A. Cortical microtubules have been observed to function as a barrier for ROP11-MIDD1 complexes in epidermal cells of Nicotiana benthamiana ${ }^{53}$, which could affect a putative reaction-diffusion mechanism between ROPGEF4, ROPGAP4 and ROP11 in metaxylem formation. To actually prove that a reaction-diffusion mechanism is functioning in xylem formation, the diffusion and reaction rates of the molecular components should be experimentally acquired. These diffusion and reaction rates can then be used in a mathematical model, which can replicate the observed patterning and predict how a pattern would change upon alteration of these rates. The ultimate proof is to verify the mathematical predictions in an experimental system and observe a predicted pattern change. Once the microtubule array is patterned it can act as a template for localised deposition for secondary cell wall material. Our results point towards a reaction-diffusion mechanism in protoxylem formation and do not provide arguments against a reaction-diffusion mechanism.

\section{Systems biology versus molecular biology}

Current interest for systems biology has triggered a renewed debate between a reductionist versus a holistic advancement of knowledge ${ }^{133}$. In biology the holistic view is characterized by encompassing complexity focussed on networks, whereas the reductionist view is characterized by simplification focussed on genepathways, biochemistry and biophysics ${ }^{134}$. Both views benefit from each other and are nonexclusive, as reductionist knowledge can be integrated in holistic networks, and the holistic approach directs towards voids in knowledge which reductionists can help to fill up. Both the holistic and the reductionist views contain paradoxes. The holistic view is as complete as the current existing knowledge base, and therefore never complete, as each new discovery can create more new questions than it answered. The reductionist view is blindfolded as it assumes connections beyond simplification are not important for a simplified system studied. The reductionist method of molecular biologists greatly improved the current understanding of biology, exemplified by the breakthrough discovery of the structure of DNA ${ }^{135}$. The great advancement of molecular biology combined with the continuous increasing computational power, allows for big data analysis 
and modelling of complex biological systems ${ }^{136}$. An example of integrating reductionist knowledge with a holistic approach is the functional network analysis of gene expression and interactions to study xylem formation in Arabidopsis ${ }^{137}$. Systems biology integrates knowledge of systems including temporal dynamics and often involves multiple spatial scales ${ }^{138}$. The search for a specific molecular component regulating patterning in xylem formation can be considered as part of a reductionists approach, whereas the study of general self-organization functions of the microtubule array involved in xylem formation fits the holistic approach. Systems biology uses computational analysis and simulation to test and generate hypothesis ${ }^{22,23}$, which is used to study microtubule dynamics in plants $58,59,87,139$. Computational analysis of minimal requirements for pattern formation reminiscent to protoxylem patterns, shows that changing the catastrophe rate in domains in a similar order as observed experimentally in vivo (chapter 3 ), results in microtubule gaps and bands in silico (chapter 4). Xylem formation is a suitable candidate for system biologists to approach in a holistic way in order to integrate molecular knowledge, genomics, proteomics, computer simulations with experimental cell biology to generate understanding at multiple spatial and temporal scales.

\section{Concluding remarks}

In this thesis we characterized the microtubule dynamics during the pattern formation of the microtubule array during protoxylem formation. To answer the main research question: how is the microtubule array able to pattern secondary cell wall deposition during protoxylem formation in plants?, we conclude that the microtubule array changes both temporally and spatially, and thereby provides a template for the cell wall deposition machinery. This microtubule template emerges by changes in microtubule dynamics. The most pronounced change is the increase of the catastrophe rate in (future) microtubule gap areas, reducing the microtubule density in these gaps over time. The microtubule bands form rings and spirals, which direct the deposition of secondary cell wall deposition during protoxylem formation in plants. In the stochastic model we show that a spatial reduction of the catastrophe rate is sufficient to recreate the experimentally observed patterned microtubule array. These spatial and temporal insights can promote the search for molecular components involved in pattern formation during protoxylem formation. Integrating current and new knowledge about molecular mechanisms, quantitative microtubule dynamics and modelling will be a fruitful endeavour to study patterned deposition of cell wall material in plant cells. 



\section{Bibliography}


1 Raven, P. H., Evert, R. F. \& Eichhorn, S. E. Biology Of Plants. 394-395, 544-546 (W.H. Freeman and Company Publishers, 2013).

2 Cosgrove, D. J. Growth of the plant cell wall. Nature 6, 850-861 (2005).

3 Emons, A. M. C. \& Mulder, B. M. How the deposition of cellulose microfibrils builds cell wall architecture. Trends in Plant Science 5, 35-40 (2000).

4 Carpita, N. C. \& Gibeaut, D. M. Structural models of primary cell walls in flowering plants: consistency of molecular structure with the physical properties of the walls during growth. The Plant Journal 3, 1-30 (1993).

5 Lindeboom, J., Mulder, B. M., Vos, J. W., Ketelaar, T. \& Emons, A. M. C. Cellulose microfibril deposition: coordinated activity at the plant plasma membrane. Journal of Microscopy 231, 192 200 (2008).

6 Sperry, J. S. Evolution of Water Transport and Xylem Structure. International Journal of Plant Sciences 164, S115-S127 (2003).

7 Bailey, I. W. C. F. p. d. J. Evolution of the Tracheary Tissue of Land Plants. American Journal of Botany 40, 4-8 (1953).

8 Carlquist, S. \& Schneider, E. L. The tracheid-vessel element transition in angiosperms involves multiple independent features: cladistic consequences. American Journal of Botany 89, 185-195 (2002).

9 Taylor, N. G., Howells, R. M., Huttly, A. K., Vickers, K. \& Turner, S. R. Interactions among three distinct CesA proteins essential for cellulose synthesis. Proceedings of the National Academy of Sciences 100, 1450-1455 (2003).

10 Taylor, N. G., Laurie, S. \& Turner, S. R. Multiple Cellulose Synthase Catalytic Subunits Are Required for Cellulose Synthesis in Arabidopsis. The Plant Cell 12, 2529-2539 (2000).

11 Taylor, N. G., Scheible, W. R., Cutler, S., Somerville, C. R. \& Turner, S. R. The irregular xylem3 locus of Arabidopsis encodes a cellulose synthase required for secondary cell wall synthesis. Plant Cell 11, 769-780 (1999).

12 Turner, S. R. \& Somerville, C. R. Collapsed xylem phenotype of Arabidopsis identifies mutants deficient in cellulose deposition in the secondary cell wall. Plant Cell 9, 689-701 (1997).

$13 \mathrm{Oda}$, Y. \& Fukuda, H. Secondary cell wall patterning during xylem differentiation. Current opinion in plant biology 15, 38-44 (2012).

14 Pesquet, E., Lloyd, C. \& Liu, B. Microtubules, MAPs and Xylem Formation. The Plant Cytoskeleton 2 277-306 (Springer New York, 2011).

15 Kenrick, P. \& Crane, P. R. Water-Conducting Cells in Early Fossil Land Plants: Implications for the Early Evolution of Tracheophytes. Botanical Gazette 152, 335-356 (1991).

16 Tarnita, C. E. et al. A theoretical foundation for multi-scale regular vegetation patterns. 541, 398401 (2017).

17 Kretschmer, S. \& Schwille, P. Pattern formation on membranes and its role in bacterial cell division. Current Opinion in Cell Biology 38, 52-59 (2016).

18 Karsenti, E. Self-organization in cell biology: a brief history. Nat Rev Mol Cell Biol 9, 255-262 (2008).

19 Tabony, J. Historical and conceptual background of self-organization by reactive processes. Biology of the Cell 98, 589-602 (2006).

20 Turing, A. M. The Chemical Basis of Morphogenesis. Philosophical Transactions of the Royal Society of London. Series B, Biological Sciences 237, 37-72 (1952).

21 Kondo, S. \& Miura, T. Reaction-Diffusion Model as a Framework for Understanding Biological Pattern Formation. Science 329, 1616-1620 (2010).

22 Ideker, T., Galitski, T. \& Hood, L. A new approach to decoding life: systems biology. Annual review of genomics and human genetics 2, 343-372 (2001).

23 Kitano, H. Systems Biology: A Brief Overview. Science 295, 1662-1664 (2002).

24 Goddard, R. H., Wick, S. M., Silflow, C. D. \& Snustad, D. P. Microtubule Components of the Plant Cell Cytoskeleton. Plant Physiology 104, 1-6 (1994). 
25 Ledbetter, M. C. \& Porter, K. R. A "microtubule in plant cell fine structure. J Cell Biol 19, 239-250 (1963).

26 Ehrhardt, D. W. \& Shaw, S. L. Microtubule dynamics and organization in the plant cortical array. Annual review of plant biology 57, 859-875 (2006).

27 Gardner, M. K., Zanic, M. \& Howard, J. Microtubule catastrophe and rescue. Current Opinion in Cell Biology 25, 14-22 (2013).

28 Dixit, R. \& Cyr, R. The cortical microtubule array: from dynamics to organization. Plant Cell 16, 2546-2552 (2004).

29 Horio, T. \& Murata, T. The role of dynamic instability in microtubule organization. Front Plant Sci 5, 511 (2014).

30 Bowne-Anderson, H., Zanic, M., Kauer, M. \& Howard, J. Microtubule dynamic instability: A new model with coupled GTP hydrolysis and multistep catastrophe. Bioessays 35, 452-461 (2013).

31 Hardham, A. R. \& Gunning, B. E. S. Structure of cortical microtubule arrays in plant cells. J Cell Biol 77, 14-34 (1978).

32 Walia, A. et al. GCP-WD mediates gamma-TuRC recruitment and the geometry of microtubule nucleation in interphase arrays of Arabidopsis. Curr Biol 24, 2548-2555 (2014).

33 Chan, J., Sambade, A., Calder, G. \& Lloyd, C. Arabidopsis cortical microtubules are initiated along, as well as branching from, existing microtubules. Plant Cell 21, 2298-2306 (2009).

34 Dixit, R. \& Cyr, R. Encounters between Dynamic Cortical Microtubules Promote Ordering of the Cortical Array through Angle-Dependent Modifications of Microtubule Behavior. The Plant Cell Online 16, 3274-3284 (2004).

35 Wasteneys, G. O. Microtubule organization in the green kingdom: chaos or self-order? Journal of Cell Science 115, 1345-1354 (2002).

36 Gardiner, J. The Evolution and Diversification of Plant Microtubule-Associated Proteins. The Plant Journal, 219-229 (2013).

37 Wieczorek, M., Bechstedt, S., Chaaban, S. \& Brouhard, G. J. Microtubule-associated proteins control the kinetics of microtubule nucleation. 17, 907-916 (2015).

38 Paredez, A. R., Somerville, C. R. \& Ehrhardt, D. W. Visualization of Cellulose Synthase Demonstrates Functional Association with Microtubules. Science 312, 1491-1495 (2006).

39 Crowell, E. F. et al. Pausing of Golgi Bodies on Microtubules Regulates Secretion of Cellulose Synthase Complexes in Arabidopsis. The Plant Cell 21, 1141-1154 (2009).

40 Gutierrez, R., Lindeboom, J. J., Paredez, A. R., Emons, A. M. \& Ehrhardt, D. W. Arabidopsis cortical microtubules position cellulose synthase delivery to the plasma membrane and interact with cellulose synthase trafficking compartments. Nature cell biology 11, 797-806 (2009).

41 Lucas, J. \& Shaw, S. L. Cortical microtubule arrays in the Arabidopsis seedling. Current opinion in plant biology 11, 94-98 (2008).

42 McFarlane, H. E., Döring, A. \& Persson, S. The Cell Biology of Cellulose Synthesis. Annual review of plant biology 65, 69-94 (2014).

43 Diotallevi, F. \& Mulder, B. The Cellulose Synthase Complex: A Polymerization Driven Supramolecular Motor. Biophysical Journal 92, 2666-2673 (2007).

44 Lindeboom, J. J. et al. A Mechanism for Reorientation of Cortical Microtubule Arrays Driven by Microtubule Severing. Science 342 (2013).

45 Kubo, M. et al. Transcription switches for protoxylem and metaxylem vessel formation. Genes \& Development 19, 1855-1860 (2005).

46 Mao, G., Buschmann, H., Doonan, J. H. \& Lloyd, C. W. The role of MAP65-1 in microtubule bundling during Zinnia tracheary element formation. Journal of Cell Science 119, 753-758 (2006).

47 Falconer, M. M. \& Seagull, R. W. Xylogenesis in tissue culture: Taxol effects on microtubule reorientation and lateral association in differentiating cells. Protoplasma 128, 157-166 (1985).

48 Demura, T. et al. Visualization by comprehensive microarray analysis of gene expression programs during transdifferentiation of mesophyll cells into xylem cells. Proceedings of the National Academy of Sciences 99, 15794-15799 (2002). 
49 Pesquet, E., Korolev, A. V., Calder, G. \& Lloyd, C. W. The Microtubule-Associated Protein AtMAP70-5 Regulates Secondary Wall Patterning in Arabidopsis Wood Cells. Current Biology 20, 744-749 (2010).

50 Derbyshire, P. et al. Proteomic Analysis of Microtubule Interacting Proteins over the Course of Xylem Tracheary Element Formation in Arabidopsis. The Plant Cell 27, 2709-2726 (2015).

51 Yamaguchi, M. et al. VASCULAR-RELATED NAC-DOMAIN6 and VASCULAR-RELATED NACDOMAIN7 Effectively Induce Transdifferentiation into Xylem Vessel Elements under Control of an Induction System. Plant Physiology 153, 906-914 (2010).

52 Watanabe, Y. et al. Visualization of cellulose synthases in Arabidopsis secondary cell walls. Science 350, 198-203 (2015).

53 Oda, Y. \& Fukuda, H. Initiation of Cell Wall Pattern by a Rho- and Microtubule-Driven Symmetry Breaking. Science 337, 1333-1336 (2012).

54 Oda, Y. \& Fukuda, H. Rho of Plant GTPase Signaling Regulates the Behavior of Arabidopsis Kinesin13A to Establish Secondary Cell Wall Patterns. The Plant Cell Online 25, 4439-4450 (2013).

55 Oda, Y., lida, Y., Kondo, Y. \& Fukuda, H. Wood Cell-Wall Structure Requires Local 2D-Microtubule Disassembly by a Novel Plasma Membrane-Anchored Protein. Current Biology 20, 1197-1202 (2010).

56 Vukasinovic, N. et al. Microtubule-dependent targeting of the exocyst complex is necessary for xylem development in Arabidopsis. The New phytologist 213, 1052-1067 (2017).

57 Oda, Y. \& Fukuda, H. Spatial organization of xylem cell walls by ROP GTPases and microtubuleassociated proteins. Current opinion in plant biology 16, 743-748 (2013).

58 Tindemans, S. H., Hawkins, R. J. \& Mulder, B. M. Survival of the Aligned: Ordering of the Plant Cortical Microtubule Array. Phys. Rev. Lett. 104, 058103 (2010).

59 Deinum, E. E., Tindemans, S. H. \& Mulder, B. M. Taking directions: the role of microtubule-bound nucleation in the self-organization of the plant cortical array. Phys Biol 8, 056002 (2011).

60 Wightman, R. \& Turner, S. R. The roles of the cytoskeleton during cellulose deposition at the secondary cell wall. The Plant Journal 54, 794-805 (2008).

61 Kondepudi, D. K. Sensitivity of chemical dissipative structures to external fields: Formation of propagating bands. Physica A: Statistical Mechanics and its Applications 115, 552-566 (1982).

62 Kondepudi, D. K. \& Prigogine, I. Sensitivity of nonequilibrium systems. Physica A: Statistical Mechanics and its Applications 107, 1-24 (1981).

63 Tabony, J., Glade, N., Papaseit, C. \& Demongeot, J. Microtubule Self-organisation and its Gravity Dependence Advances in Space Biology and Medicine 8, 19-58 (Elsevier, 2002).

64 Skagen, E. B. \& Iversen, T.-H. Simulated weightlessness and hyper-g results in opposite effects on the regeneration of the cortical microtubule array in protoplasts from Brassica napus hypocotyls. Physiol Plantarum 106, 318-325 (1999).

65 Skagen, E. B. \& Iversen, T. H. Effect of simulated and real weightlessness on early regeneration stages of Brassica napus protoplasts. In vitro cellular \& developmental biology. Plant : journal of the Tissue Culture Association 36, 312-318 (2000).

66 Bollhöner, B., Prestele, J. \& Tuominen, H. Xylem cell death: Emerging understanding of regulation and function. Journal of experimental botany 63, 1081-1094 (2012).

67 Wasteneys, G. O. \& Fujita, M. Establishing and maintaining axial growth: wall mechanical properties and the cytoskeleton. J Plant Res 119, 5-10 (2006).

68 Baskin, T. I. On the alignment of cellulose microfibrils by cortical microtubules: a review and a model. Protoplasma 215, 150-171 (2001).

69 Baskin, T. I. \& Gu, Y. Making parallel lines meet: transferring information from microtubules to extracellular matrix. Cell adhesion \& migration 6, 404-408 (2012).

70 Pickett-Heaps, J. D. The effects of colchicine on the ultrastructure of dividing plant cells, xylem wall differentiation and distribution of cytoplasmic microtubules. Developmental Biology 15, 206236 (1967). 
71 Hepler, P. K. \& Fosket, D. E. The role of microtubules in vessel member differentiation in Coleus. Protoplasma 72, 213-236 (1971).

72 Oda, Y., Mimura, T. \& Hasezawa, S. Regulation of Secondary Cell Wall Development by Cortical Microtubules during Tracheary Element Differentiation in Arabidopsis Cell Suspensions. Plant Physiology 137, 1027-1036 (2005).

73 Schindelin, J., Rueden, C. T., Hiner, M. C. \& Eliceiri, K. W. The Image J ecosystem: An open platform for biomedical image analysis. Mol. Reprod. Dev. 82, 518-529 (2015).

74 Updegraff, D. M. Semimicro determination of cellulose inbiological materials. Analytical Biochemistry 32, 420-424 (1969).

75 Rotman, B. \& Papermaster, B. W. Membrane properties of living mammalian cells as studied by enzymatic hydrolysis of fluorogenic esters. Proceedings of the National Academy of Sciences of the United States of America 55, 134-141 (1966).

76 Lucas, J. R. \& Shaw, S. L. MAP65-1 and MAP65-2 promote cell proliferation and axial growth in Arabidopsis roots. The Plant Journal 71, 454-463 (2012).

77 Kosetsu, K., de Keijzer, J., Janson, M. E. \& Goshima, G. MICROTUBULE-ASSOCIATED PROTEIN65 is essential for maintenance of phragmoplast bipolarity and formation of the cell plate in Physcomitrella patens. Plant Cell 25, 4479-4492 (2013).

78 Turner, S., Gallois, P. \& Brown, D. Tracheary element differentiation. Annual review of plant biology 58, 407-433 (2007).

79 Schindelin, J. et al. Fiji: an open-source platform for biological-image analysis. Nature methods 9 , 676-682 (2012).

80 Mulder, B. M. Microtubules interacting with a boundary: Mean length and mean first-passage times. Phys. Rev. E 86, 011902 (2012).

81 Yamaguchi, M. et al. VASCULAR-RELATED NAC-DOMAIN 7 directly regulates the expression of a broad range of genes for xylem vessel formation. The Plant Journal 66, 579-590 (2011).

82 Winter, D. et al. An "Electronic Fluorescent Pictograph" browser for exploring and analyzing large-scale biological data sets. PLOS ONE 2, e718 (2007).

83 Cyr, R. J. Microtubules in Plant Morphogenesis: Role of the Cortical Array. Annual Review of Cell Biology 10, 153-180 (1994).

84 Desai, A. \& Mitchison, T. J. MICROTUBULE POLYMERIZATION DYNAMICS. Annual review of cell and developmental biology 13, 83-117 (1997).

85 Sedbrook, J. C. MAPs in plant cells: delineating microtubule growth dynamics and organization. Current opinion in plant biology 7, 632-640 (2004).

86 Sedbrook, J. C. \& Kaloriti, D. Microtubules, MAPs and plant directional cell expansion. Trends in Plant Science 13, 303-310 (2008).

87 Hawkins, R. J., Tindemans, S. H. \& Mulder, B. M. Model for the orientational ordering of the plant microtubule cortical array. Phys. Rev. E 82, 011911 (2010).

88 Deinum E.E., Tindemands S.H. \& Mulder B.M Taking directions: the role of microtubule-bound nucleation in the self-organization of the plant cortical array. Physical Biology 8, 056002 (2011).

89 Hoson, T. \& Soga, K. in International review of cytology Vol. Volume 229 209-244 (Academic Press, 2003).

90 Tabony, J., Glade, N., Demongeot, J. \& Papaseit, C. Biological Self-Organization by Way of Microtubule Reaction-Diffusion Processest. Langmuir 18, 7196-7207 (2002).

91 Surrey, T., Nédélec, F., Leibler, S. \& Karsenti, E. Physical Properties Determining Self-Organization of Motors and Microtubules. Science 292, 1167-1171 (2001).

92 Bastiaens, P., Caudron, M., Niethammer, P. \& Karsenti, E. Gradients in the self-organization of the mitotic spindle. Trends in cell biology 16, 125-134 (2006).

93 Gov, N. S., McSharry, S. S. \& Beitel, G. J. Three-ring circus without a ringmaster: Self-organization of supracellular actin ring patterns during epithelial morphogenesis. Proceedings of the National Academy of Sciences 112, 8521-8522 (2015). 
94 Tee, Y. H. et al. Cellular chirality arising from the self-organization of the actin cytoskeleton. Nature cell biology 17, 445-457 (2015).

95 Saiki, M. et al. Cellular basis for the automorphic curvature of rice coleoptiles on a threedimensional clinostat: possible involvement of reorientation of cortical microtubules. Journal of Plant Research 118, 199-205 (2005).

96 Hoson, T., Matsumoto, S., Soga, K. \& Wakabayashi, K. Cortical microtubules are responsible for gravity resistance in plants. Plant Signal Behav 5, 752-754 (2010).

97 Hoson, T. et al. Growth stimulation in inflorescences of an Arabidopsis tubulin mutant under microgravity conditions in space. Plant biology (Stuttgart, Germany) 16 Suppl 1, 91 -96 (2014).

98 Jost, A.-I. K., Hoson, T. \& Iversen, T.-H. The Utilization of Plant Facilities on the International Space Station-The Composition, Growth, and Development of Plant Cell Walls under Microgravity Conditions. Plants 4, 44-62 (2015).

99 de Micco, V., Aronne, G., Joseleau, J. P. \& Ruel, K. Xylem development and cell wall changes of soybean seedlings grown in space. Annals of botany 101, 661-669 (2008).

100 Kwon, M., Bedgar, D. L., Piastuch, W., Davin, L. B. \& Lewis, N. G. Induced compression wood formation in Douglas fir (Pseudotsuga menziesii) in microgravity. Phytochemistry 57, 847-857 (2001).

101 Sieberer, B. J., Kieft, H., Franssen-Verheijen, T., Emons, A. M. \& Vos, J. W. Cell proliferation, cell shape, and microtubule and cellulose microfibril organization of tobacco BY-2 cells are not altered by exposure to near weightlessness in space. Planta 230, 1129-1140 (2009).

$102 \mathrm{Hu}, \mathrm{Z}$. \& Lutkenhaus, J. Topological regulation of cell division in Escherichia coli involves rapid pole to pole oscillation of the division inhibitor MinC under the control of MinD and MinE. Molecular Microbiology 34, 82-90 (1999).

103 Kondepudi, D. K. \& Prigogine, I. Sensitivity on nonequilibrium chemical systems to gravitational field. Advances in Space Research 3, 171-176 (1983).

104 van Loon, J. J. et al. The large diameter centrifuge, LDC, for life and physical sciences and technology. Life in Space for Life on Earth, 663, 92 (2008).

105 Borst, A. G. \& van Loon, J. J. W. A. Technology and Developments for the Random Positioning Machine, RPM. Microgravity Science and Technology 21, 287-292 (2008).

106 van Loon, J. J. W. A. Some history and use of the random positioning machine, RPM, in gravity related research. Advances in Space Research 39, 1161-1165 (2007).

107 Ruyters, G. \& Braun, M. Plant biology in space: recent accomplishments and recommendations for future research. Plant biology (Stuttgart, Germany) 16 Suppl 1, 4-11 (2014).

108 Green, P. B. Pathways Of Cellular Morphogenesis. J Cell Bio 27, 343-363 (1965).

109 Baskin, T. I. Anisotropic expansion of the plant cell wall. Annual review of cell and developmental biology 21, 203-222 (2005).

110 Mueller, S. C. \& Brown, R. M., Jr. Evidence for an intramembrane component associated with a cellulose microfibril-synthesizing complex in higher plants. J Cell Bio/ 84, 315-326 (1980).

111 Kimura, S. et al. Immunogold Labeling of Rosette Terminal Cellulose-Synthesizing Complexes in the Vascular Plant Vigna angularis. The Plant Cell 11, 2075-2085 (1999).

112 Bringmann, M. et al. POM-POM2/cellulose synthase interacting1 is essential for the functional association of cellulose synthase and microtubules in Arabidopsis. Plant Cell 24, 163-177 (2012).

$113 \mathrm{Zhu}, \mathrm{C}$. et al. The fragile Fiber1 kinesin contributes to cortical microtubule-mediated trafficking of cell wall components. Plant Physiol 167, 780-792 (2015).

114 Kong, Z. et al. Kinesin-4 Functions in Vesicular Transport on Cortical Microtubules and Regulates Cell Wall Mechanics during Cell Elongation in Plants. Mol Plant 8, 1011-1023 (2015).

115 Laskowski, M. J. Microtubule orientation in pea stem cells: a change in orientation follows the initiation of growth rate decline. Planta 181, 44-52 (1990).

116 Ueda, K. \& Matsuyama, T. Rearrangement of cortical microtubules from transverse to oblique or longitudinal in living cells of transgenic Arabidopsis thaliana. Protoplasma 213, 28-38 (2000). 
117 Nick, P., Bergfeld, R., Schäfer, E. \& Schopfer, P. Unilateral reorientation of microtubules at the outer epidermal wall during photo- and gravitropic curvature of maize coleoptiles and sunflower hypocotyls. Planta 181, 162-168 (1990).

118 Pickett-Heaps, J. D. Incorporation of radioactivity into wheat xylem walls. Planta 71, 1-14 (1966).

119 Pickett-Heaps, J. D. The effects of colchicine on the ultrastructure of dividing plant cells, xylem wall differentiation and distribution of cytoplasmic microtubules. Developmental Biology 15, 206236 (1967).

120 Lindeboom, J. J. Pattern formation of cortical microtubules and cellulose microfibrils, Wageningen University, (2012).

121 Yang, Z. Cell polarity signaling in Arabidopsis. Annual review of cell and developmental biology 24, 551-575 (2008).

122 Fu, Y., Gu, Y., Zheng, Z., Wasteneys, G. \& Yang, Z. Arabidopsis interdigitating cell growth requires two antagonistic pathways with opposing action on cell morphogenesis. Cell 120, 687-700 (2005).

123 Stoppin-Mellet, V., Gaillard, J. \& Vantard, M. Functional evidence for in vitro microtubule severing by the plant katanin homologue. The Biochemical journal 365, 337-342 (2002).

124 Nakamura, M. \& Hashimoto, T. A mutation in the Arabidopsis $\gamma$-tubulin-containing complex causes helical growth and abnormal microtubule branching. Journal of Cell Science 122, 22082217 (2009).

125 Nakamura, M., Ehrhardt, D. W. \& Hashimoto, T. Microtubule and katanin-dependent dynamics of microtubule nucleation complexes in the acentrosomal Arabidopsis cortical array. 12, 1064-1070 (2010).

126 Chan, J., Calder, G. M., Doonan, J. H. \& Lloyd, C. W. EB1 reveals mobile microtubule nucleation sites in Arabidopsis. Nature cell biology 5, 967-971 (2003).

127 Mimori-Kiyosue, Y., Shiina, N. \& Tsukita, S. The dynamic behavior of the APC-binding protein EB1 on the distal ends of microtubules. Current Biology 10, 865-868 (2000).

128 Lindeboom, J. J. et al. Cortical Microtubule Arrays Are Initiated from a Nonrandom Prepattern Driven by Atypical Microtubule Initiation. Plant Physiology 161, 1189-1201 (2013).

129 Endo, H. et al. Multiple Classes of Transcription Factors Regulate the Expression of VASCULARRELATED NAC-DOMAIN7, a Master Switch of Xylem Vessel Differentiation. Plant and Cell Physiology 56, 242-254 (2015).

130 Ohtani, M. et al. A NAC domain protein family contributing to the regulation of wood formation in poplar. The Plant Journal 67, 499-512 (2011).

131 Schuetz, M. et al. Laccases direct lignification in the discrete secondary cell wall domains of protoxylem. Plant Physiology 166, 798-807 (2014).

132 Oda, Y., lida, Y., Nagashima, Y., Sugiyama, Y. \& Fukuda, H. Novel Coiled-Coil Proteins Regulate Exocyst Association with Cortical Microtubules in Xylem Cells via the Conserved Oligomeric Golgi-Complex 2 Protein. Plant and Cell Physiology 56, 277-286 (2015).

133 Mazzocchi, F. Complexity and the reductionism-holism debate in systems biology. WIREs Syst Biol Med 4, 413-427 (2012).

134 Gatherer, D. So what do we really mean when we say that systems biology is holistic? BMC Systems Biology 4, 22 (2010).

135 Watson, J. D. \& Crick, F. H. C. Molecular Structure of Nucleic Acids: A Structure for Deoxyribose Nucleic Acid. Nature 171, 737-738 (1953).

136 Kitano, H. Computational systems biology. Nature 420, 206 (2002).

137 Davin, N. et al. Functional network analysis of genes differentially expressed during xylogenesis in soc1ful woody Arabidopsis plants. The Plant Journal 86, 376-390 (2016).

138 O'Malley, M. A. \& Dupré, J. Fundamental issues in systems biology. Bioessays 27, 1270-1276 (2005).

139 Deinum, E. E. \& Mulder, B. M. Modelling the role of microtubules in plant cell morphology. Current opinion in plant biology 16, 688-692 (2013). 



\section{Summary}

Plant cells are surrounded by cell walls. Cell walls provide strength to protect and shape the cells they surround. Structural strength is particularly important in the water conducting tissue of the xylem. The xylem tissue is responsible for water and nutrient transport from root to shoot. Water and nutrient transport is driven by water evaporation at the leaves, generating a negative pressure in the tubular xylem vessels. Patterned cell wall reinforcements are deposited during the development of these cells that function in counteracting the negative pressure. The cell wall reinforcements are deposited in a diverse number of patterns. Secondary cell wall thickenings in the form of rings and spirals provide physical strength, while allowing elongation of the surrounding tissue in the newly grown plant parts. Plant cell walls consist of a mixture of polysaccharides, forming an intricate network with cellulose microfibrils as the main load-bearing component. The cortical microtubule array dictates the direction and location of newly polymerised cellulose microfibrils in primary cell wall deposition. In this thesis, I focus on understanding if and how microtubule dynamics control the patterning of secondary cell wall position.

In chapter 1 I introduce protoxylem formation in plant cells, how plant cells can form a pattern, microtubule dynamics and the main research question: which self-organizing properties of microtubules change to generate a microtubule pattern and how is this pattern translated to a patterned cell wall deposition during protoxylem formation? In chapter 2 we focus on the reorganization of the cortical microtubule array into high density microtubule bands and low density microtubule gaps preceding patterned cell wall deposition. VND7 is the master regulator of protoxylem formation in Arabidopsis (Arabidopsis thaliana) which we use to reproducibly induce protoxylem formation in cortical cells. We characterize the VND7-inducible protoxylem differentiation program by determining the time scale of the microtubule array reorganization, the deposition of secondary cell wall thickenings and programmed cell death. To study the function of the microtubule array during protoxylem differentiation we define an early, mid and late protoxylem differentiation stage. In the early stage, the microtubule array gets patterned into microtubule bands perpendicular to the growth axis, with occasional microtubules deviating from the main perpendicular orientation in between microtubule bands. In the mid stage, microtubule bands become more compact and fewer microtubules deviate from the microtubule band orientation. In the late stage, we observe splitting and disintegration of microtubule bands and detect the first deposition of secondary cell wall material. To study the function of the microtubule array, we apply microtubule depolymerising or stabilizing drugs during protoxylem 
formation. Destabilization of microtubules during protoxylem formation results in a reduced percentage of cells that deposit banded cell wall thickenings. In addition, we observe irregular patterned secondary cell wall thickenings when microtubules are destabilized during protoxylem formation. Treatment with the microtubule stabilizing drug taxol accelerates the onset of patterned cell wall deposition, suggesting that microtubule stability controls the onset of patterned cell wall deposition. These findings indicate that the microtubule array is essential for patterned deposition of banded cell wall thickenings in protoxylem formation and may have a role in initiation of cell wall deposition.

In chapter 3 we determine which microtubule dynamics change during the reorganization of the microtubule array into high density microtubule bands and low density microtubule gaps. Microtubules can grow and shrink, the switch between the growing and shrinking state of a microtubule is defined as a catastrophe and from shrinking to growing as a rescue. Microtubules switching stochastically between growing and switching states are considered dynamic instable. We measured the microtubule dynamicity parameters growth velocity, shrink velocity, rescue rate and catastrophe rate which are important for the self-organizational capability of the microtubule array. We show that the rescue and catastrophe rate increases in the microtubule gap area compared to the microtubule band area. The measured microtubule dynamicity parameters allow for the calculation of the mean microtubule length. In microtubule gap regions the calculated mean length is lower compared to the calculated mean length in microtubule band regions. These results indicate that local changes in the rescue and catastrophe rate are responsible for the generation of microtubule bands and gaps over time during protoxylem formation. In chapter $\mathbf{4}$ we stochastically simulate microtubules to test if a spatial manipulation of the catastrophe rate is sufficient to generate the typical protoxylem pattern of microtubule bands and gaps. We show that a 2 -fold increase of the catastrophe rate is sufficient to generate microtubule bands and gaps. This strongly suggests that a spatial manipulation of the catastrophe rate, as observed in plant cells during protoxylem formation (chapter 3 ) is sufficient to generate a patterned microtubule array.

The reorganization of one stable organization of the microtubule array into another stable organization is likely to involve a non-equilibrium organization during the transition. During these stages, the array may display an increased sensitivity to external stimuli. In chapter 5 we investigate if gravity is a factor able to influence the reorganization of the microtubule array during protoxylem formation. In chapter 2 we show that the microtubule array is essential for correct deposition of secondary cell wall thickenings, thus we consider the patterned cell wall thickenings as a readout for the microtubule reorganization during protoxylem formation. We 
compare the band width and band distance of secondary cell wall thickenings of plant cells which transdifferentiated under simulated microgravity or hypergravity conditions. The secondary cell wall pattern did not alter significantly between control conditions and simulated microgravity or hypergravity conditions, indicating that the measured parameters are not affected by the experimental conditions that we tested. Thus, altered gravity conditions do not sufficiently affect microtubule patterning to change patterned deposition of secondary cell wall thickenings during protoxylem formation.

In chapter 6, firstly I discuss the plant cell wall deposition machinery, next, how this machinery is controlled via molecular mechanisms, and finally, how patterns of secondary cell wall thickenings are formed during protoxylem formation. Taken together, the results presented in this thesis provide insight in when, where and how the microtubule array is modified during protoxylem differentiation. This knowledge contributes to a better understanding of the organizational principles of the microtubule array in general and is an important aspect in understanding pattern formation during protoxylem formation. 



\section{Acknowledgements}

My thesis is done. Now it is time to try to thank all the people who supported me along the way. Tijs, thank you for being there for me in times of struggle and success. The freedom you gave me was sometimes difficult, as I wasn't sure if I was on the right track, but in the long run you provided the support which I needed to finish this thesis. Thank you for guiding me to find my own path within the scientific community and to greatly improve my writing skills by patiently providing feedback on my texts. Jasper, thank you for supporting me 'from a distance', as your expertise differed from the direct methods I used in this thesis. Still, I always felt welcome at Physical Chemistry and Soft Matter for your advice, even when you became the chair holder of this group. Marcel, thank you for discussion and advice along the way at the chair group of Cell Biology. Bela, thank you for guiding me into the world of programming. This new direction within my PhD provided the change I required to keep motivated in times when my plant experiments did not yield results quick enough to satisfy my aspiration. And thanks for supporting me during the last year of my thesis with feedback, advice and motivation. Jack, thank you for assisting me into the world of gravitational experiments.

Juliane and Olya, thank you for providing a welcome environment in the lab and at the microscope room to start my PhD. Aniek, thanks for chocolate notes and lovely pies at social events, all the organisational effort for pub quizzes and WEdays, holidays together and happy kiwi-breaks. Jeroen, where to start, well at the beginning. The first course of our BSc Biology in 2015, we worked together on a report about bioterrorism. From there onwards our study-path coincides very closely with shared courses in our BSc and MSc related to plant biology, and then even a PhD at Cell Biology. Thanks for all the discussions concerning the scientific world, the struggles in the PhD work and the uncertain future which will be. Thank you to all the students who joined me during my PhD project: Miranda, Levine, Kim, Laura, Lisa, Axel, Cosmas \& Elysa. Elysa, you became a colleague and I want to thank you for bringing life back to the group when we most needed it. Norbert, thanks for the support on the microscope front and providing delicious additions to pot-lucks with Christmas and other occasions. Henk, thanks for keeping our labs up and running and assisting with IT-problems and for taking me to the slaughterhouse early in the morning on our mission to acquire tubulin. Hannie, thanks for returning to our lab as a teacher showing me that an education career at the university is possible. Marie-José, thanks for all the administrative support and of course organizational support for the Christmas dinner and the lab trips. André, you are my hero, the way you teach is a great example for me, with patience and passion. It was wonderful to be taught by you as a student and to stand next to you 
as an assistant during various courses. Thanks to the whole group of Cell Biology including visitors and students joining our group over the years. Han, thank you for all the conversations and dinners we shared.

From Molecular Biology and Plant Developmental Biology I want to thank Adam, Rik, TingTing, Arjan, Huchen, Sabine \& Bandan and all the others for sharing coffee breaks, kiwi breaks, WE-days and lab trips. You were great neighbours. To support the welfare of EPS PhD students I joined the EPS PhD council, which also proofed to be a welcome social addition to my PhD time in Wageningen. Thanks Hanna, Setareh, Jordi, Tatjana, Suraj, Lotte and Lot for a great time together. My time was spent mostly in the Radix with my colleagues in plant science, but on the other side of town I had big group of colleagues at Physical Chemistry and Soft Matter. I have seen a generation of $\mathrm{PhD}$ students graduate amongst which I met some great people during parties, BBQ's and jam sessions. Armando, thanks for being the social spider within the group and introducing me to all your colleagues and friends. Yunus, food, friends, music, discussion, all we shared and more. Thanks for the great time together. Dmitry, the first time I met you, you danced, after that, the chance of seeing you dance was small. I hope you will find the moments in life when dance is good way to express yourself. Christian, thanks for all the great talks we shared. Mervé, thanks for sticking around almost all the way to the end of my PhD time in Wageningen. You graduated before me. I still remember the great time we shared discussing almost everything, and yes, you satisfied my curiosity a bit. But as scientist we both know; there is always another question we can ask! Thanks for organizing the PCC PhD trip to the UK: Natalia, Maarten, Hanne, Wolf \& Gosia. By joining I got to know you better as people and as scientists: Jeroen, Jan Maarten, Ilse, Jan Bart, Maria, Inge \& Marcel, Joshua \& Frans. Joanne, you started as a student at Cell Biology and then became a PCC colleague. Thanks for the good times together shared at both groups. Thanks Sabine, Johan, Natalia, Gosia, Josie, Mara, Anita and to all the others at PCC. Thanks to my new colleagues while finalising this thesis: Ruud, Lennart, Philippe, Milou, Andrada and all the others.

Thanks to those who showed that music and science is a great combination: Marten Scheffer, Bert Lotz, Martien Cohen Stuart \& Tim Hofmeester. Without music my PhD would not be possible. Thank you to all the musicians with whom I played over the years: Alexandre, Armando, Yunus, Otto, Jelle, Roeland, Samira, Chrissie, Tim, Jolanda, Wouter, Jasper, Bram, Gonda, Harsh, Jelle, Samira, Dmitry, Julia, Coen, Todd, Frank, Wout, Mali, Stefan, Suzanne, Aljosha, Daan, Santos, Yvette, Piers, Veronica, Roan, Artur, Sander, Zdeněk, Floriane, Bert, Judica, Corentin, Emilie, Pascal, Fabian, Mara, Martin and all the others.

Tim, you are special. A friend in life and in music. Let's us live and play together, even now you move to Sweden for (hopefully only) a couple of years. 
Anne-mie, bedankt voor je desem, dezelfde cultuur is nog steeds levend en ik bak er nog regelmatig brood van. Jan-vos, de manier hoe jij les gaf is geweldig, bedankt voor de motivatie om de celbiologie van planten in te duiken. Eva, thanks for assisting me in the world of microtubule modelling. René, meeting you was very important to me. Finally I could speak with somebody who truly understood my daily work as we both performed similar experiments. Staffan, thanks for our discussions. Zdeněk, thanks for sharing dinners and music together. Pádraic, it was great meeting you dancing in Utrecht on the streets, to meet later again during an evacuation of the Radix as we both worked on plants for our PhD projects. Julio, what I remember mostly is your smile.

Friends from Droevendaal, thanks for making Droef an amazing place to live: Alexander, Evelien, Hanne, Stefan, Evelien, Ellen, Jeroen, Esther, Thomas, Roos, Rozemarijn, Peter, Kawire, Renske, Doris, Diederik, Leander, Lucas, Merel, Lucia, Luc, Mart, Mira, Fabian, Lukas, Sophie and all the others.

Familie is belangrijk voor mij, en daarom bedank ik Elin, omdat je zo klein bent, Stijn, omdat je zo stoer bent, Jack, omdat je zo'n lage stem hebt (tegenwoordig), Annemiek, omdat je zo lief bent, Els, omdat je mijn liefste jongste oudere zus bent (um), Lieke, omdat jij mijn voorbeeld bent hoe om te gaan met kinderen, Martje, omdat ik trots op je ben. Lieve mama, bedankt voor alles. Ik ben blij dat je mijn mama bent. Gerard, bedankt voor alle steun en vragen die je altijd weer stelde, en positieve uitspraken over mijn voortgang van mijn PhD project. Ook Ettiene en Marieke bedankt. Eligius \& Marjon, Jos \& Septimia, bedankt dat ik bij jullie kon logeren als ik weer eens een conferentie in Amsterdam had. De rest van mijn familie, allemaal bedankt!

Steven, graag wandel ik elk jaar met jou over de Veluwe. Merlijne, blijf de kunst maken die je maakt, ik vind het mooi. Céline, lang hebben we elkaar niet gesproken maar in mijn gedachte ben je er nog altijd bij. Bauke, van spelletjes tot wandeltocht in Noorwegen, ik ben blij dat je mijn vriend bent. Sylvia, samen begonnen we aan de studio biologie, bostochten en pannenkoeken volgde en logeerpartijen in Berlijn. Durk, de gesprekken met jou gaan heerlijk diep, ik kijk uit naar ons volgende kopje koffie. Michelle, bedankt voor alle tijd samen en de steun die jij mij gaf tijdens mijn PhD

Floor, omdat het toch bijzonder is om in zoveel dankwoorden te staan, bedankt voor de thee, het fijne samenwonen en zoveel meer. Succes met de volgende tien! Jelle, buurman, muzikant, paranimf, huisgenoot, wandelgenoot en meer, bedankt. Lieve Yvette, fijn dat je mijn leven ingedanst bent. 



\section{Curriculum vitae}

Kris Joppe van 't Klooster was born in Arnhem, The Netherlands, on April 1987. He attended the high school 'Olympus College' in Arnhem. For his BSc degree in biology he attended Wageningen University, The Netherlands, from 2005-2009. This was followed from 2009-2012 with his MSc in biology with as specialization: plants. In his MSc thesis he studied the secondary cellulose synthase proteins in plant cells at the Laboratory of Plant Cell Biology, Wageningen University. For his MSc internship he studied parasitic weeds at the CGIAR research centre AfricaRice in Tanzania, in collaboration with the Centre for Crop Systems Analysis, Wageningen University.

In 2012 he started as a PhD student with Prof. dr. Bela Mulder,Dr. ir. Tijs Ketelaar, Laboratory of Cell Biology and Prof. dr. ir. J. van der Gucht, Laboratory of Physical Chemistry and Soft Matter at Wageningen University on the patterning of plant cell wall deposition by cortical microtubules in plant cells.

Since his last year of his BSc Kris is involved in teaching at Wageningen University. Starting as a student assistant during his BSc and MSc, then in between his MSc and his PhD project as a teaching assistant and finally as part of his PhD project. For 1.5 year he was part of the PhD council of the graduate school Experimental Plant Sciences.

Free time is for the most important thing in life: music. 



\section{Education Statement of the Graduate School Experimental Plant Sciences}

Issued to: Kris van 't Klooster

Date: 18 October 2017

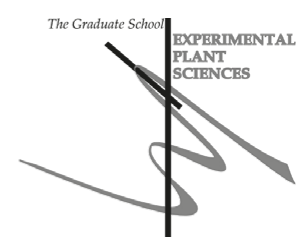

Group: Laboratory of Cell Biology \& Physical Chemistry and Soft Matter

University: Wageningen University \& Research

\section{1) Start-up phase}

date

- First presentation of your project

Title: Spatial regulation of cell wall deposition during xylem formation

- Writing or rewriting a project proposal

- Writing a review or book chapter

- MSc courses

- Laboratory use of isotopes

\section{2) Scientific Exposure}

Subtotal Start-up Phase

1.5 credits*

\section{EPS PhD student days}

EPS PhD student day, Wageningen, NL date

EPS PhD student day, Leiden, NL

30 Dec 2012

EPS PhD studen day, 'Get2Gether', Soest, NL

\section{EPS theme symposia}

EPS theme 1 symposium 'Developmental Biology of Plants', Wageningen, NL

19 Jan 2012

EPS theme 2 symposium 'Interactions between Plant and Biotic Agents',

02 Feb 2012

Wageningen, NL

EPS theme 1 symposium 'Developmental Biology of Plants', Leiden, NL

17 Jan 2013

EPS theme 1 symposium 'Developmental Biology of Plants', Wageningen, NL

24 Jan 2014

EPS theme 1 symposium 'Developmental Biology of Plants', Leiden, NL

08 Jan 2015

- National meeting (e.g. Lunteren days) and other national platforms

KNAW Biophysics Meeting, Amsterdam, NL

31 Jan 2012

Annual meeting 'Experimental Plant Sciences', Lunteren, NL

02-03 Apr 2012

Annual Dutch Meeting on Cellular and Molecular Biophysics, Veldhoven, NL

01-02 Oct 2012

KNAW Biophysics Meeting, Amsterdam, NL

22 Jan 2013

Annual meeting 'Experimental Plant Sciences', Lunteren, NL

22-23 Apr 2013

Annual meeting 'Experimental Plant Sciences', Lunteren, NL

14-15 Apr 2014

Dutch Biophysics, Veldhoven, NL

29-30 Sep 2014

Annual meeting 'Experimental Plant Sciences', Lunteren, NL

13 Apr 2015

- Seminars (series), workshops and symposia

Seminar Peter van Gisbergen

12 Jan 2012

Seminar Gijsje Koenderink

02 Mar 2012

Seminar Erwin Peterman

13 Apr 2012 
Seminar Florian Hollandt

04 May 2012

Workshop: New developments in plant endomembrane biology

15 Jun 2012

Workshop ISLSWG: Plant Biology in Space (Freiburg)

01-03 Aug 2012

Seminar Paul van der Schoot

26 Oct 2012

Mini-symposium (Chun-Ming Liu, Sidney Shaw, Dolf Weijers, Viktor Zárský)

13 Nov 2012

Seminar Larry Griffin

16 Nov 2012

Seminar Andrew Sugden, editor science

08 Feb 2013

EPS flying seminar Detlef Weigel

27 Feb 2013

Seminar Richard Lenski

15 Mar 2013

Soft Matter Symposium - Farewell Prof. Martien Cohen Stuart

26 Apr 2013

Seminar WEES: Marten Scheffer

23 May 2013

Plant Developmental Biology Symposium

14 Oct 2013

Workshop Scientific Integrity - AMOLF

26 Nov 2013

Workshop Scientific Integrity (only PhDs and Postdocs) - AMOLF

28 Nov 2013

Nexus-Institute Conference - The Triumph of Science

Wageningen University PhD Symposium

01 Dec 2013

10 Dec 2013

Biophysics Seminar Marcel Reuter

11 Dec 2013

Systems Biology Seminar - Henrik Jönsson

12 Dec 2013

EPS flying seminar - Noah Whiteman

17 Jul 2014

Seminar Phong Tran

04 Sep 2014

Nexus-institute Conference - War \& Peace

20 Sep 2014

Seminar Yara Sanchez

13 Nov 2014

Mini-symposium (Erik Limpens, Tijs Ketelaar, Eric Schranz)

24 Nov 2014

Seminar Jonne Rodenburg

01 Dec 2014

EPS flying seminar - Yves van Peer

03 Feb 2015

Seminar Dimitrios Vavylonis

17 Apr 2015

Systems Biology Seminar - Pieter Rein ten Wolde

12 May 2015

Seminar - Martin Jinek

02 Jun 2015

Nexus-institute Conferece - Waiting for the Barbarians

14 Nov 2015

\section{Seminar plus}

EPS Flying Seminar - Gero Steinberg

05 Jun 2015

\section{International symposia and congresses}

Gordon Research Conference on Plant and Fungal Cytoskeleton, USA

12-17 Aug 2012

EMBO meeting 2013, Amsterdam, NL

21-24 Sep 2013

European Plant Science Retreat 2014, Amsterdam, NL

01-04 Jul 2014

RMS Botanical Microscopy Meeting, Exeter, UK

20-23 Apr 2015

\section{Presentations}

Poster: Spatial regulation of cell wall deposition during xylem formation (Lunteren)

Poster: Spatial regulation of cell wall deposition during xylem formation (Veldhoven)

02-03 Apr 2012

01-02 Oct 2012 
Talk: Plant Developmental Biology Symposium

14 Oct 2013

Talk: Visit Staffan Perssons group MPI molecular plantfysiology (Golm,

08 Sep 2014

Germany)

Poster: Towards understanding microtubule reorganization during xylem

29-30 Sep 2014 formation (Veldhoven)

Talk: RMS Botanical Microscopy Meeting, Exeter (UK)

20 Apr 2015

Talk: PhD Excursion UK, University of Cambridge, Cambridge (UK)

29 Apr 2015

Talk: PhD Excursion UK, University of Oxford, Oxford (UK)

30 Apr 2015

Talk: Current topics in Plant Biotechnology, MSc Seminar (WUR)

07 Jun 2016

\section{IAB interview}

\section{Excursions}

Rijk Zwaan, De Lier, NL

27 Sep 2013

GeneTwister, In2Care, Wageningen, NL

19 Sep 2014

PhD Excursion UK 2015

Subtotal Scientific Exposure

29.1 credits* $^{*}$

\section{3) In-Depth Studies}

date

\section{EPS courses or other PhD courses}

Matlab Course: Image Processing Toolbox ${ }^{\mathrm{TM}}$, Eindhoven, NL

27-28 Nov 2012

EMBL Modeling Cellular Processes in Space and Time Course, France

10-17 Oct 2015

\section{Journal club}

Literature discussion group CLB

$2012-2013$

- Individual research training

Java Modelling, AMOLF - Amsterdam

Nov-Dec 2013

Subtotal In-Depth Studies

4) Personal development

6.6 credits $^{*}$

Skill training courses

EPS Expectations Day: Inspiration \& Creativity

01 Feb 2013

EPS Expectations Day: Communication \& Ethics

28 Mar 2014

Career Orientation, Wageningen, NL

08-29 Oct 2014

PhD Peer Consultation, Wageningen, NL

Sep 2014-May

2015

\section{Organisation of PhD students day, course or conference}

EPS Get2Gether

29-30 Jan 2015

\section{Membership of Board, Committee or PhD council}

Membership of PhD council

Sep 2013-Jan

2015

Subtotal Personal Development

5.8 credits $^{\star}$

Herewith the Graduate School declares that the PhD candidate has complied with the educational requirements set by the Educational Committee of EPS which comprises of a minimum total of 30 ECTS credits

*A credit represents a normative study load of 28 hours of study. 
The research conducted in this thesis was financially supported by the SRON space research program with project number GO-MG/15, which is financed by the Netherlands Organization for Scientific Research (NWO). Advanced microscopy experiments were performed with support of the Shared Research Facility of Wageningen University.

Financial support from Wageningen University for printing this thesis is gratefully acknowledged.

Layout design: Iliana Boshoven-Gkini | AgileColor.com

Cover design: Floor Hofman

Printed: Ridderprint on FSC-certified paper 


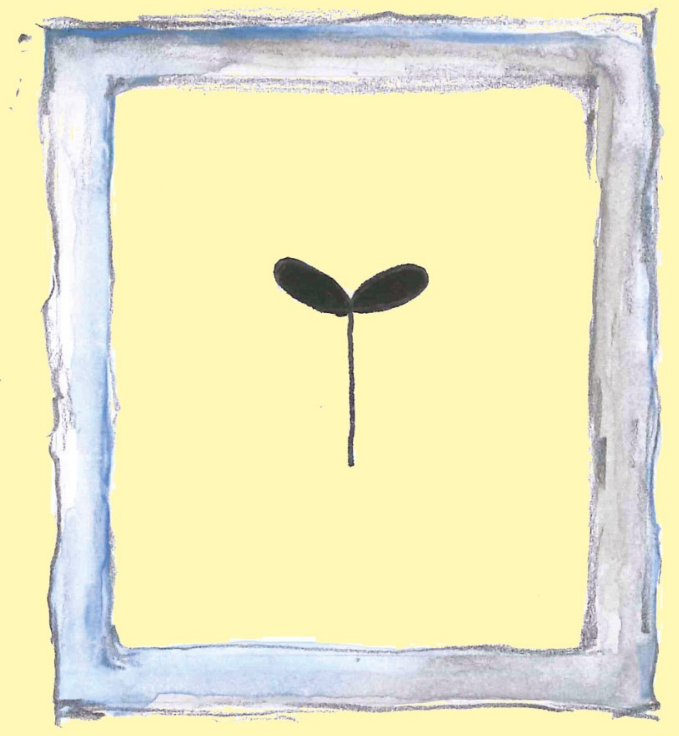

